\title{
AstRoMap European Astrobiology Roadmap
}

\author{
Gerda Horneck, ${ }^{1,2, *}$ Nicolas Walter, ${ }^{3, *}$ Frances Westall, John Lee Grenfell, William F. Martin, ${ }^{6}$ Felipe Gomez, \\ Stefan Leuko, ${ }^{2, *}$ Natuschka Lee, ${ }^{8,9}$ Silvano Onofri, ${ }^{10}$ Kleomenis Tsiganis, ${ }^{11}$ Raffaele Saladino, ${ }^{12}$ \\ Elke Pilat-Lohinger, ${ }^{13}$ Ernesto Palomba, ${ }^{1,{ }^{*}}$ Jesse Harrison, ${ }^{15}$ Fernando Rull, ${ }^{16}$ Christian Muller, ${ }^{17, *}$ \\ Giovanni Strazzulla, ${ }^{18}$ John R. Brucato, ${ }^{19}$ Petra Rettberg, ${ }^{2, *}$ and Maria Teresa Capria ${ }^{14, *}$
}

\begin{abstract}
The European AstRoMap project (supported by the European Commission Seventh Framework Programme) surveyed the state of the art of astrobiology in Europe and beyond and produced the first European roadmap for astrobiology research. In the context of this roadmap, astrobiology is understood as the study of the origin, evolution, and distribution of life in the context of cosmic evolution; this includes habitability in the Solar System and beyond. The AstRoMap Roadmap identifies five research topics, specifies several key scientific objectives for each topic, and suggests ways to achieve all the objectives. The five AstRoMap Research Topics are

- Research Topic 1: Origin and Evolution of Planetary Systems

- Research Topic 2: Origins of Organic Compounds in Space

- Research Topic 3: Rock-Water-Carbon Interactions, Organic Synthesis on Earth, and Steps to Life

- Research Topic 4: Life and Habitability

- Research Topic 5: Biosignatures as Facilitating Life Detection
\end{abstract}

It is strongly recommended that steps be taken towards the definition and implementation of a European Astrobiology Platform (or Institute) to streamline and optimize the scientific return by using a coordinated infrastructure and funding system. Key Words: Astrobiology roadmap-Europe-Origin and evolution of life-Habitability—Life detection—Life in extreme environments. Astrobiology 16, 201-243.

${ }^{1}$ European Astrobiology Network Association.

${ }^{2}$ Institute of Aerospace Medicine, German Aerospace Center (DLR), Köln, Germany.

${ }^{3}$ European Science Foundation, Strasbourg, France.

${ }^{4}$ Centre National de la Recherche Scientifique-Centre de Biophysique Moléculaire, Orleans, France.

${ }^{5}$ Institute for Planetary Research, German Aerospace Center (DLR), Berlin, Germany.

${ }^{6}$ Institute of Molecular Evolution, Heinrich-Heine University of Düsseldorf, Düsseldorf, Germany.

${ }^{7}$ INTA Centre for Astrobiology, Torrejón de Ardoz, Madrid, Spain.

${ }^{8}$ Department of Ecology and Environmental Science, Umeå University, Umeå, Sweden.

${ }^{9}$ Department of Microbiology, Technical University München, München, Germany.

${ }^{10}$ Department of Ecological and Biological Sciences, University of Tuscia, Viterbo, Italy.

${ }^{11}$ Department of Physics, Section of Astrophysics, Astronomy and Mechanics, Aristotle University of Thessaloniki, Thessaloniki, Greece.

${ }^{12}$ Department of Agrobiology and Agrochemistry, University of Tuscia, Viterbo, Italy.

${ }^{13}$ Institute of Astrophysics, University of Vienna, Vienna, Austria.

${ }^{14}$ INAF-Institute for Space Astrophysics and Planetology, Rome, Italy.

${ }^{15}$ Department of Microbiology and Ecosystem Science, University of Vienna, Vienna, Austria.

${ }^{16}$ Department of Condensed Matter Physics, Crystallography and Mineralogy, Valladolid University, Valladolid, Spain.

${ }^{17}$ B.USOC, Brussels, Belgium.

${ }^{18}$ INAF-Catania Astrophysical Observatory, Catania, Italy.

${ }^{19}$ INAF-Arcetri Astrophysical Observatory, Florence, Italy.

*Members of the AstRoMap project team.

(C) Gerda Horneck, et al., 2016; Published by Mary Ann Liebert, Inc. This Open Access article is distributed under the terms of the Creative Commons Attribution Noncommercial License (http://creativecommons.org/licenses/by-nc/4.0/) which permits any noncommercial use, distribution, and reproduction in any medium, provided the original author(s) and the source are credited. 


\section{Table of Contents}

1. Introduction

1.1. The project AstRoMap within the Framework Programme for Research and Innovation (FP7) of the European Union

1.2. The European astrobiology environment and landscape in Europe 203

1.3. Setting the scene: timeline and astrobiology concepts 205

2. The Astrobiology Roadmap for Europe 207

3. Research Topic 1: Origin and Evolution of Planetary Systems 208

3.1. State of the art 208

3.2. Key objectives 209

3.3. Approach to achieve the key objectives 211

3.4. European strengths and needs 213

4. Research Topic 2: Origins of Organic Compounds in Space 214

4.1. State of the art

4.2. Key objectives 214

4.3. Approach to achieve the key objectives 216

4.4. European strengths and needs 218

5. Research Topic 3: Rock-Water-Carbon Interactions, Organic Synthesis on Earth, and Steps to Life 218

5.1. State of the art 218

5.2. Key objectives 218

5.3. Approach to achieve the key objectives 220

5.4. European strengths and needs 221

6. Research Topic 4: Life and Habitability 222

6.1. State of the art 222

6.2. Key objectives 223

6.3. Approach to achieve the key objectives 225

6.4. European strengths and needs 227

7. Research Topic 5: Biosignatures as Facilitating Life Detection 227

7.1. State of the art 227

7.2. Key objectives 228

7.3. Approach to achieve the key objectives 230

7.4. European strengths and needs 232

8. Conclusions and Recommendations 233

8.1. Cross-cutting issues of relevance 233

8.2. Towards a better coordination of astrobiology research in Europe- the need for a pan-European platform 234

Acknowledgments

References

Abbreviations Used

\section{AstRoMap Project Partners}
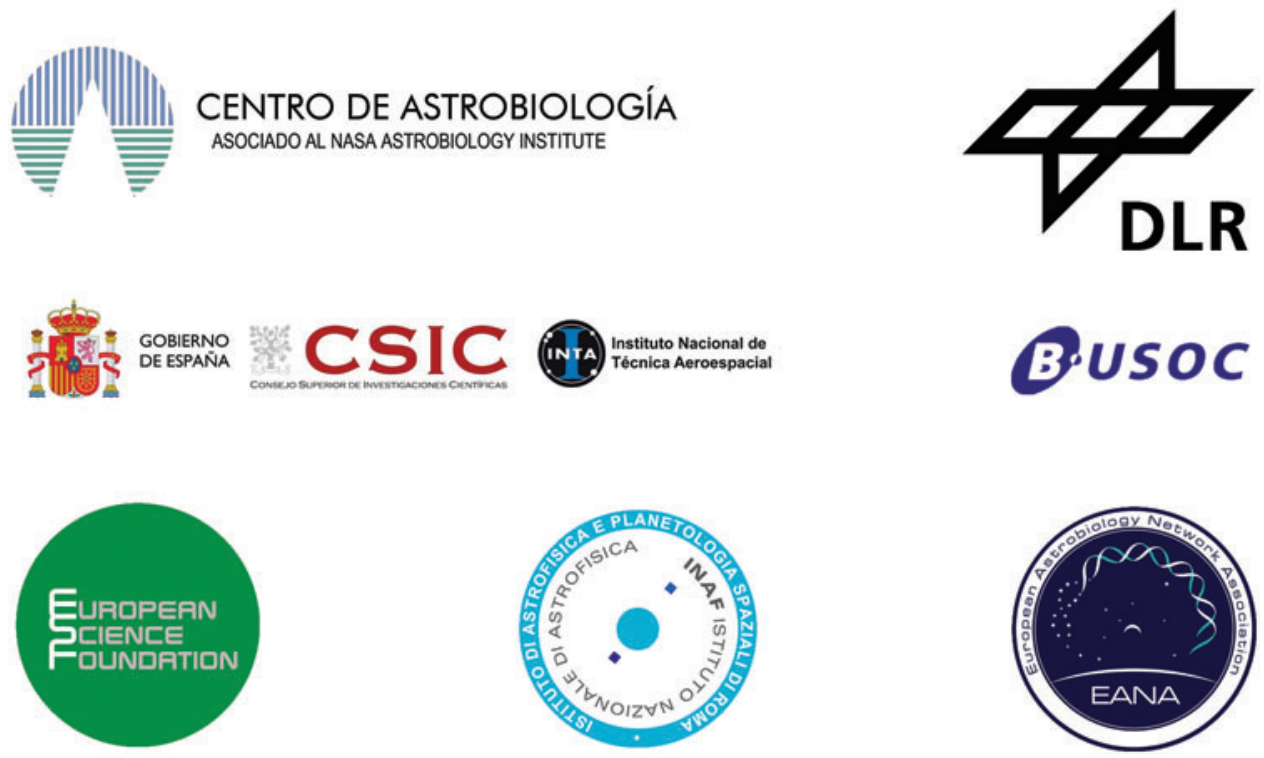


\section{Introduction}

\subsection{The project AstRoMap within the Framework Programme for Research and Innovation (FP7) of the European Union}

A STROBIOLOGY IS a broad research domain that encompasses wide areas of the scientific landscape. This maturing field cuts across many disciplines ranging from prebiotic chemistry to geomicrobiology, atmospheric sciences, and astronomy. Astrobiology is not only diverse in terms of disciplines. It also traverses a very wide spectrum of spatial and temporal scales: from the molecular level to ecosystems and planetary systems, at scales ranging from Earth's (sub)surface to planetary objects detected thousands of lightyears away, and from understanding the origins of life to its future evolution and destiny. By nature, astrobiology touches on some of the more fundamental societal questions: What is life? How did it start? Is it present beyond Earth? It is also relevant to more earthbound concerns such as the evolution of ecosystems under growing environmental pressure or the development of new and innovative (bio)technologies.

Active for the period 2013-2015 and supported by the European Union through its Seventh Framework Programme for Research and Innovation (FP7), the AstRoMap project has undertaken the task of developing a roadmap for European research in astrobiology, taking into consideration its multidisciplinary nature and putting forward overarching priority research topics and key scientific objectives in a structured and coherent way. The AstRoMap partner organizations were the Instituto Nacional de Técnica Aeroespacial Centro de Astrobiología (INTA-CAB, Spain), the European Science Foundation (ESF, France), Deutsches Zentrum für Luft- und Raumfahrt (DLR, Germany), the Belgian User Support and Operations Centre (B-USOC, Belgium), the Istituto Nazionale di Astrofisica (INAF, Italy) and the European Astrobiology Network Association (EANA, Europe). In the context of the AstRoMap Roadmap, astrobiology is understood as the study of the origin, evolution, and distribution of life in the context of cosmic evolution; this includes habitability in the Solar System and beyond (see http:// www.astromap.eu).

This document presents the outcome of this effort. It results from the integration of inputs from the scientific community collected via expert workshops as well as online consultations. Overall, more than 45 European experts were involved in the definition of the AstRoMap Roadmap.

The AstRoMap European Astrobiology Roadmap is structured around five core research topics that represent interrelated roadmap building blocks. Each research topic is broken down into several key objectives (and sub-objectives) that put forward more detailed priority areas to be addressed in the future. For each research topic, the roadmap also suggests potential achievements in the short (within the next decade), medium (within the next two decades), and long (beyond 20 years) term; it also addresses the specificities of the European landscape in terms of strengths and needs relevant to each topic.

\subsection{The European astrobiology environment and landscape in Europe}

1.2.1. European Space Agency (ESA). At ESA, astrobiology research themes are covered by the Directorate of
Science and Robotic Exploration (D/SRE) and the Directorate of Human Spaceflight and Operations (D/HSO). ESA's mandatory space science program funds the development and construction of the spacecraft and the launch and operation of space missions but not the development and construction of scientific instrumentation nor the exploitation of scientific data or ground-based laboratory research. Support for the latter needs to be obtained from national organizations. ESA's optional space research program includes work on robotic and human exploration in the context of three potential destinations: low-Earth orbit (LEO), the Moon, and Mars.

Astrobiology (originally termed "exobiology") was recognized by ESA as a future research area as early as 1996. A study group (the ESA exobiology team) was established to survey the state of research in exobiology and related fields and to make recommendations to ESA on the nature of future "Search for Life" actions elsewhere in the Solar System (Brack et al., 1999a, 1999b). This first European initiative to embrace astrobiology resulted in a comprehensive exobiological view of the Solar System by exploring the four following topics:

- Chemical evolution in the Solar System;

- Limits of life under extreme conditions;

- Morphological and biochemical signatures of extraterrestrial life: utility of terrestrial analogues;

- Potential sites for extraterrestrial life.

This far-seeing initiative strongly influenced the definition and setup of the Aurora program as an optional program, ESA's strategic framework for space exploration. Aurora commenced in January 2002 by setting out a strategy over the next 30 years for Europe's robotic and human exploration of Mars, the Moon, and even beyond to asteroids. ExoMars, the first European Mars mission dedicated to astrobiology, was one of the program's flagship missions.

The very ambitious plans of the program also envisaged two Mars sample return missions, a robotic outpost on Mars, and a possible human mission to the Moon and a human mission to Mars in the horizon 2025-2030. However, the Aurora program was not sufficiently backed by high-level political commitment and was later terminated.

Although the Aurora program was cancelled, its first flagship mission, ExoMars, is in the process of being implemented. The ExoMars mission is structured in two elements: the first element, launched on March 14, 2016, includes the ExoMars Trace Gas Orbiter (TGO, atmospheric gas analysis, in particular methane mapping) and the Schiaparelli lander (Entry, Descent, and Landing Demonstrator Module and meteorological payload). The 2018 element will carry the ExoMars rover with its suite of astrobiology experiments (the Pasteur payload) intended to assess the habitability of past and present Mars and detect signatures of extinct life (Vago et al., 2006, 2015; Parnell et al., 2007; Vago and Kminek, 2007).

In the early 2000s, in addition to its exploration strategy and to follow up on the previous plan (Horizon 2000 plus), ESA developed the framework and the backbone of its mandatory science program for the period 2015-2025. The 
Cosmic Vision program was issued in October 2005 (ESA, 2005). Among the scientific questions subdivided into topics where important progress was expected, the first theme of the program is strongly related to astrobiology, although the term astrobiology is not mentioned: What are the conditions for planet formation and the emergence of life? This theme is divided into three topics:

- From gas and dust to stars and planets. Place the Solar System into the overall context of planet formation, aiming at comparative planetology. Map the birth of stars and planets by peering into the highly obscured cocoons where they form.

- From exoplanets to biomarkers. Search for planets around stars other than the Sun, looking for biomarkers in their atmospheres, and image them.

- Life and habitability in the Solar System. Explore in situ the surface and subsurface of the solid bodies in the Solar System most likely to host-or have hostedlife. Explore the environmental conditions that make life possible.

The Cosmic Vision program was used to delineate the boundary conditions of the subsequent call for scientific missions open to the community. As of mid-2015 six missions have been selected and are in various stages of development; half of those missions have relevance to astrobiology. Two missions, CHaracterising ExOPlanet Satellite (CHEOPS, launch planned in 2017) and PLAnetary Transits and Oscillations of stars (PLATO 2.0, launch planned in 2024), are dedicated to the detection of exoplanets; and one large mission, JUpiter ICy moons Explorer (JUICE, launch planned in 2028), will explore the jovian system, flying by three of its icy moons (Callisto, Europa, and Ganymede) before orbiting Jupiter.

In 2013, ESA's High-level Science Policy Advisory Committee (HISPAC) published a report (HISPAC, 2013) in which four major science themes were recommended as scientific challenges that should be tackled by the agency in the next decades, beyond the already defined scientific objectives being currently implemented in the various programs. In this report, the third theme specifically addresses "Life in the Universe," and "Astrobiology" is outlined as a case study.

In addition to the ExoMars and Cosmic Vision program, astrobiology is mentioned and fully integrated in ESA's optional European Life and Physical Sciences in Space program (ELIPS). Started in 2001, ELIPS allows research teams to have access to the European Columbus laboratory on the International Space Station (ISS) as well as to other ISS platforms (including exposure facilities) and ground-based platforms and facilities (for example, drop towers, sounding rockets, radiation facilities). ELIPS allowed the development and use of the EXPOSE facility; this facility, mounted on the outside of the ISS, allows exposure of biological samples and organic compounds to a combination of space environmental factors (full spectrum of extraterrestrial solar electromagnetic radiation, cosmic radiation, vacuum, freezing/thawing, and microgravity) to investigate their resistance and adaptation to extreme space and (simulated) planetary conditions. The EXPOSE facility was deployed on three occasions:

- in 2008-2009 for the EXPOSE-E mission: seven experiments-18 months of exposure (EXPOSE-E, 2012)
- in 2009-2011 for the EXPOSE-R mission: nine experiments-22 months of exposure (EXPOSE-R, 2015)

- in 2014-2016 for the EXPOSE-R2 mission: four experiments-planned for 18 months of exposure.

1.2.2. Astrobiology in the context of the European Union. One of the main research and technology development programs of the European Union is Horizon 2020; this program succeeds the Seventh Framework Programme (FP7) and covers the period 2014-2020. Horizon 2020 addresses all aspects of science and technology development and is structured around biannual work programs and annual calls.

It is expected that Horizon 2020 will dedicate approximately $1.4 \mathrm{~B} €$ to space activities, $5 \%$ of which will target space sciences. In effect, most Horizon 2020 space-related call topics are targeted towards developing appropriate space technologies and services and fostering European industry competitiveness. In fact, space is positioned under the heading "Leadership in enabling and industrial technologies" of the program.

In the first call of Horizon 2020 (2014), the topic Space exploration and science (with a total budget of $4 \mathrm{M} €$ ) focused on Mars data evaluation and on the definition of a European sample curation facility for sample return missions (see http://www.euro-cares.com), in addition to dedicated technology developments. In the second call (2015), this same topic focused on scientific exploitation of astrophysics, comets, and planetary data with a budget of $1.5 \mathrm{M} €$, as well as a topic addressing international cooperation in the context of planetary protection with $1 \mathrm{M} €$ budget.

1.2.3. The European astrobiology community. In addition to the programmatic arena, the European astrobiology community benefits from networking and interaction platforms at national, European, and international levels. At the European level, the European Astrobiology Network Association (EANA) was established in 2001 and provides a platform and a forum for the astrobiology community in Europe (19 European countries are represented) and beyond (see eana-net.eu). For 15 years, EANA has organized an annual workshop on astrobiology, providing a tangible forum for interaction and collaboration, and is very active in the area of education through the $\mathrm{ABC}-\mathrm{Net}$ lecture courses, a live teleteaching program performed in cooperation with ESA, interconnecting several European universities (Horneck and Rettberg, 2007) (see http://eana-net.eu/education .html).

Launched in 2014 for a period of 4 years, the Cooperation in Science and Technology (COST) Action ORIGINS (TD1308) is a European network involving researchers from 29 European countries and focuses on scientific questions related to the origins and evolution of life on Earth and habitability of other planets. It provides a common platform for interdisciplinary interactions and coordination of nationally funded investigations (see http:// life-origins.com).

Building on the success of two previous European Unionfunded projects and structured around a platform gathering together more than 60 research institutions (all signatories to the Europlanet Memorandum of Understanding), the 
European Planetary Network (Europlanet) has developed and is implementing the 2020 Research Infrastructure project (EPN2020-RI) supported by the Horizon 2020 Research Infrastructure program. EPN2020-RI is a 4-year, $10 \mathrm{M} €$ initiative that started in September 2015 (see http:// www.europlanet-2020-ri.eu) and brings together 33 partners from 18 European countries. Its main objective is to provide a pan-European Union infrastructure dedicated to planetary sciences. EPN2020-RI will (i) network state-ofthe-art research facilities and provide access to them; (ii) organize access to planetary analog sites on Earth; and (iii) set up a "virtual observatory" for planetary science, making high-level data accessible to the community. EPN2020-RI will provide the community with a platform that will catalyze and facilitate multidisciplinary research for European planetary scientists.

Other ongoing significant initiatives include the European Astrobiology Campus (EAC-an Erasmus+ strategic partnership) that will be active for the period 2014-2016 (see http://astrobiology-campus.eu/about-eac-2). The EAC brings together 12 European universities and science education organizations. This initiative will provide multidisciplinary education in astrobiology to students as well as training to lecturers. Another objective of the EAC is to raise awareness of astrobiology by delivering information, material, and tools to the general public.

European early-career astrobiologists are also organizing their networking through the Astrobiology Graduates in Europe (AbGradE) association (see http://eana-net.eu/AbGradE/ about.html). Since 2014, this association organizes symposia and workshops during which master classes, keynote lectures, and scientific sessions are provided and held.

In 2013, the AstRoMap project organized a survey of the European astrobiology science community; this survey was later published in Acta Astronautica (Horneck et al., 2015). Compilation of the data provided by 105 European investigators allowed for the creation of a detailed profile of the community involved in astrobiology in Europe. This community is mostly composed of planetologists interested in habitability and life detection, as well as biologists interested in extremophiles on Earth. It also involves astrophysicists, physicists, chemists, geologists, and astronomers. The community makes use of laboratory studies and simulation facilities and includes direct investigations in Earth extreme environments and in space. Connected to astrobiology, this community is also interested in earthbound issues, in particular global change, biosphere sustainability, and humanities and social sciences.

Astrobiology is perceived in different ways by the various communities involved, and the national astrobiology landscapes differ significantly from one country to another. Nevertheless, building on the first European astrobiology experiments supported through ELIPS, on the creation and activities of EANA in the early 2000s, and on the various national astrobiology societies, the burgeoning implication and strength of the European astrobiologists' community is apparent. The pan-European initiatives presented above are new and innovative. They support this community in a coherent manner at various career stages and provide visibility and stability to its researchers. This demonstrates that the European astrobiology community has reached a level of maturity that enables ongoing pro- jects to be built up, data from past projects to be exploited, and researchers across the board to engage in better coordination and consolidation of astrobiological projects. Thus, it is very timely to put forward and suggest a number of scientific priorities that should be addressed at the European level.

\subsection{Setting the scene: timeline and astrobiology concepts}

1.3.1. Timeline-from formation of stellar systems to life. As one of the main questions of astrobiology, the origin and evolution of life on Earth has attracted great interest from many different fields of expertise. The presence of life has changed the panorama of primitive Earth. Indeed, one of the difficulties concerning study of primitive Earth, upon which life arose, comes from the fact that life itself changed its environment. Life is a physicochemical process that takes inputs from the exterior in order to auto-organize its interior, modifying the environment in the process. Several crucial events took place along the timeline of Earth's history up to the period when environmental conditions allowed an explosion in biodiversity resulting in the multitude of species colonizing all habitable environments of Earth, as we know it today (Fig. 1). Earth's timeline starts about 4600 million years ago, when the planet formed from the accretion disc revolving around a young star. Even during this very early phase, complex organic molecules necessary for later life may already have formed in the protoplanetary disc of gas and dust grains. The formation of the Moon had important consequences from an astrobiological point of view. Very soon after its consolidation, 4500 million years (or 4.5 billion years) ago, and according to the giant impact hypothesis, the Moon was formed when planet Earth and another hypothesized planet collided (Hartmann and Davis, 1975). It has been suggested that the newly formed Moon stabilized Earth's fluctuating axis of rotation due to its gravitational pull and, hence, the environmental conditions in which life formed. Meanwhile, several other events took place during the formation of the gas giants and rocky planets.

Owing to lack of evidence (all rocks older than about 4 Ga have been recycled), there is much debate about the geology of early Earth, but it is known that primitive continents (like oceanic plateaus) formed during the Hadean between about 4.4 and $4.0 \mathrm{Ga}$. True continents did not appear until much later, about $3 \mathrm{Ga}$ (Dhuime et al., 2015). The earliest direct signatures of primitive life date back to about 3.5 Ga (Walsh, 1992; Westall, 2011), although life must have arisen much earlier because by $3.5 \mathrm{Ga}$ it was already diversified and included anoxygenic photosynthesizers (Byerly et al., 1986; Hofmann et al., 1999; Tice and Lowe, 2004; Westall and Southam, 2006). Moreover, there is a tantalizing indication of its presence at $3.8 \mathrm{Ga}$ (Ohtomo et al., 2013). However, it is with the rise of photosynthesis and, especially, oxygenic photosynthesis that life started to modify the environment. This absolutely critical evolution has not yet been precisely dated but occurred probably after $3.0 \mathrm{Ga}$. Its signatures in the form of giant stromatolites were already in place around the world by $2.7-2.6 \mathrm{Ga}$ (Nisbet and Wilks, 1988; Eriksson and Altermann, 1998), but it is only as of $2.4 \mathrm{Ga}$ with the "Great Oxidation Event" (Holland, 
ENVIRONMENT

LIFE

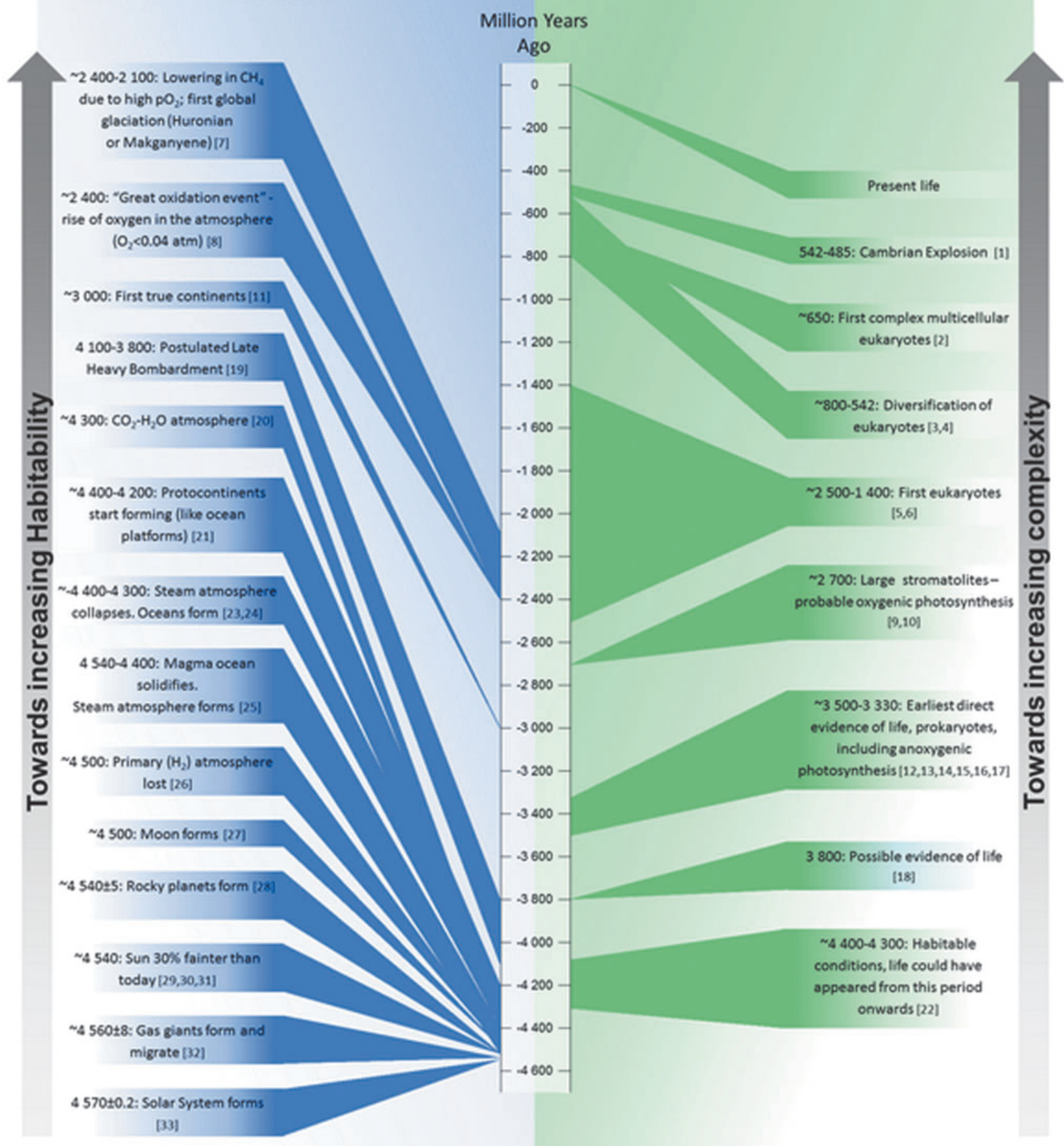

FIG. 1. Timeline in million years from the formation of the Solar System to the appearance and evolution of life on Earth. [1] Cloud, 1948; [2] Grosberg and Strathmann, 2008; [3] Knoll, 2011; [4] Mills et al., 2014; [5] Javaux, 2007; [6] Parfrey et al., 2011; [7] Melezhik, 2006; [8] Holland, 2006; [9] Nisbet and Wilks, 1988; [10] Altermann and Wotherspoon, 1995; [11] Dhuime et al., 2015; [12] Byerly et al., 1986; [13] Hofmann et al., 1999; [14] Tice and Lowe, 2004; [15] Westall and Southam, 2006; [16] Walsh, 1992; [17] Westall, 2011; [18] Ohtomo et al., 2013; [19] Tera et al., 1974; [20] Holland, 1984; [21] Van Kranendonk et al., 2015; [22] Westall, 2012; [23] Elkins-Tanton, 2012; [24] Wilde et al., 2001; [25] Lebrun et al., 2013; [26] Zahnle et al., 1988; [27] Kleine et al., 2005; [28] Dalrymple, 2001; [29] Sagan and Mullen, 1972; [30] Gough, 1981; [31] Feulner, 2012; [32] Walsh et al., 2011; [33] Baker et al., 2005. 
2006) that there was sufficient oxygen in the environment to make significant global changes-leading, for instance, to the first global glaciation (the Huronian or Makganyene glaciation, 2.4-2.1 Ga, Melezhik, 2006). The modification of the environment through the atmospheric oxygen rise allowed other organisms (with other metabolic pathways) to appear and different forms of life to evolve.

1.3.2. Approaching habitability. The concept of habitability is one of the fundamental issues for our understanding of the origin, diversity, and extent of life on Earth and our ability to identify extraterrestrial environments that are, or were, able to support life or may even support life in the future. These may include environments that allow life to emerge and enable its continued existence as well as evolution towards a higher complexity (Gershenson and Lenaerts, 2008). Therefore, in the simplest sense, a habitable environment can be identified as one that can allow the development, maintenance, and evolution of life. This ability relates either to a given time and location ("instantaneous habitability") or to the maintenance of habitable conditions over geological time ("continuous habitability"). While most habitable environments on Earth are typically characterized by the presence of life, the presence of biological activity is not a prerequisite for habitability. A habitable environment, therefore, may be either inhabited or uninhabited (e.g., Cockell et al., 2012; Harrison et al., 2013; Westall et al., 2013).

Despite the diversity of life on Earth, all organisms explored so far share several requirements, such as the availability of elements (in particular $\mathrm{C}, \mathrm{H}, \mathrm{N}, \mathrm{O}, \mathrm{P}, \mathrm{S}$, trace metals, etc.) needed for macromolecular synthesis, liquid water, energy, and appropriate physical-chemical conditions (such as temperature). The ability of an environment to sustain life can be measured in several ways, and as a result the exact definition of habitability varies between scientific fields. Today, habitability is classified into two general categories: "planetary habitability" (the ability of a planetary body to develop and sustain life) and the "habitable zone", (the zone around a star where liquid water is stable at the surface of a planetary body) (Kasting et al., 1993). Investigations of the former rely on our knowledge of extremophilic organisms and the physical-chemical limits to biological processes on Earth, providing a detailed starting point for understanding the habitability of other planetary bodies such as Mars, the icy moons of outer planets, or exoplanets. Research into the limits for life and its evolutionary potential on Earth relies on the characterization of two key parameters: (i) physiological mechanisms that underpin stress tolerance, measurements of cell division, metabolic activity, dispersal, and survival in response to different physical-chemical extremes; and (ii) evolutionary ecological principles that serve as driving forces for the coevolution of life and its environment, thereby constantly reshaping the boundaries of habitability and, thus, the conditions for the continuity and evolution of life.

Investigations of habitability at the level of entire planetary systems are based on the concept of the habitable zone. The presence of liquid water, therefore, plays a key role in investigating the habitability of extraterrestrial environments. For very distant objects, such as exoplanets, the identification of potentially habitable conditions depends on numerous factors including planetary mass, orbit, type of atmosphere, and the properties of the central star. However, the question is whether these few parameters are sufficient. The AstRoMap Roadmap intends to identify key research topics that will broaden our knowledge of habitability on all levels of organization, ranging from planetary bodies within our solar system to other planetary systems in our galaxy.

\section{The Astrobiology Roadmap for Europe}

Based on the inputs from the experts involved in AstRoMap, a roadmap has been constructed for astrobiology in Europe, which consists of five interconnected research topics to be addressed in parallel:

- Research Topic 1: Origin and Evolution of Planetary Systems

- Research Topic 2: Origins of Organic Compounds in Space

- Research Topic 3: Rock-Water-Carbon Interactions, Organic Synthesis on Earth, and Steps to Life

- Research Topic 4: Life and Habitability

- Research Topic 5: Biosignatures as Facilitating Life Detection

These five research topics reflect essential steps towards reaching the final goal: a better understanding of life and its origin and evolution within the context of cosmic evolution (Fig. 2), that is, regarding life as a cosmic phenomenon (De Duve, 1996).
Based on the scientific experience and technical capabilities available in Europe, three to five key objectives have been determined for each research topic, and approaches have been suggested as to how these objectives will be reached within the next 10 or 20 years or after that period (Chapters 3-7 and Figs. 3-7).

It is strongly recommended that this AstRoMap Roadmap be adopted by the European Union as a challenge to enhance Europe's standing as an attractive partner for international partnerships in space science and exploration. The Roadmap should be supported by cross-disciplinary research in the five Research Topics listed above and described below. This requires the establishment of a pan-European astrobiology coordination platform or European virtual astrobiology institute under the auspices of a pan-European funding organization, for example, the European Union, the European Space Agency, or the European Science Foundation (see section 8.2). 


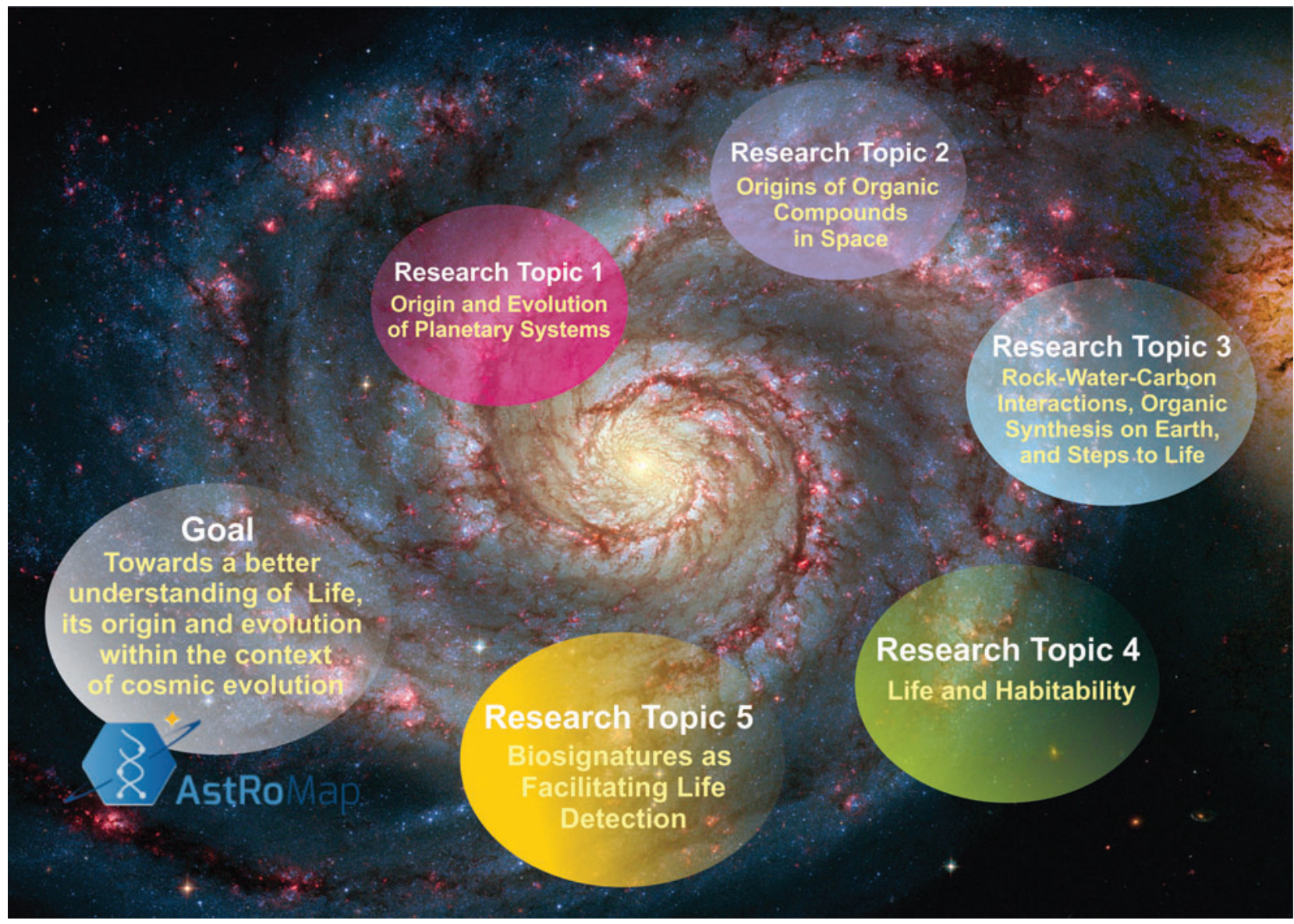

FIG. 2. The AstRoMap Roadmap: An astrobiology roadmap for Europe, consisting of five research topics to be addressed in parallel towards reaching the final goal-a better understanding of life within the context of cosmic evolution. Background picture: M51 Hubble Remix (http://apod.nasa.gov/apod/ap080614.html). Credit: S. Beckwith (STScI), Hubble Heritage Team (STScI/AURA), ESA, NASA. Additional Processing: Robert Gendler. Graphic Design: Kerstin Kopp, DLR.

\section{Research Topic 1: Origin and Evolution of Planetary Systems}

\subsection{State of the art}

Our solar system is the planetary system that is best known to us, and so far it is the only system known to host life. A wealth of data, collected over many decades through observations, space missions, and field and laboratory studies, have been used to formulate our ever-evolving theories of how Earth, the other planets, and their satellites formed and how the system has dynamically evolved towards its current state.

In the context of astrobiology, understanding the origin of water and other materials essential for life, on Earth and on other, possibly habitable environments in the Solar System, is of profound importance. The chemical composition of the first planetesimals - the building blocks of planets - and how they evolved as a function of distance from the Sun can be inferred by studying collected extraterrestrial materials and by analyzing remote sensing data of comets and asteroids. New amazing results from the ESA Rosetta space mission demonstrate the presence of organic material on the nucleus of the Jupiter-family comet 67P/Churiumov-Gerasimenko, probably formed by the interaction of ices $\left(\mathrm{H}_{2} \mathrm{O}, \mathrm{CO}_{2}, \mathrm{CO}\right)$ with solar radiation (Capaccioni et al., 2015). The measured
$\mathrm{D} / \mathrm{H}$ ratio of the comet $67 \mathrm{P}$ was found to be larger than that previously measured in comets, and this finding opens up new questions about the formation mechanisms of comets and the chemical models of the protoplanetary solar nebula (Altwegg et al., 2015). Models are necessary to explain the present Solar System architecture. These models are continuously refined by feedback both from observations of our own and other planetary systems and from laboratory simulation experiments. Nevertheless, many gaps remain. These observations should involve protoplanetary discs for which the only known species are $\mathrm{CO}, \mathrm{CN}, \mathrm{HCN}$, or $\mathrm{HCO}^{+}$, and future observations are needed to build up more robust chemistry models (Beuther et al., 2014, and references therein; Dutrey et al., 2014).

Since the observation of the first exoplanet orbiting a Sunlike star (Mayor and Queloz, 1995), astrophysicists have realized that planet formation should include orbit migration involving chaotic phases in planetary systems, which makes it difficult to reconstruct the initial configuration of the protoplanets.

Stars and planets are the end products of a complex chain of mechanisms, starting in the galactic interstellar medium where molecular cloud contraction and collapse define the 
initial conditions for the formation of stars and their planetary systems. Our understanding of how planets form around stars is far from complete and increasingly challenged by new discoveries. Recent space missions, such as COnvection ROtation and planetary Transits (CoRoT) and the US-led counterpart Kepler, have provided valuable new information on this topic and helped develop new concepts of missions such as CHEOPS and PLATO 2.0, which are slated to be launched by ESA in 2017 and 2024, respectively. As of July 2015 more than 1500 confirmed planets have been observed, both by missions and by ground-based facilities (see www.exoplanets.org).

Planets form in protoplanetary discs that surround young stars. The formation process of discs, their physical, chemical, and dynamical evolution, as well as the various steps of planetary growth and subsequent evolution, obviously lead to the wide variety of observed planetary environments. The orbital architecture of exoplanetary systems is also highly diverse: "hot" giant planets, highly compact multiplanet systems, planets around binary stars, highly eccentric orbits, and even inclined planetary systems. The mechanisms responsible for these turbulent events, their strength and duration, as well as the final dynamical configuration could be paramount for determining whether a system may host habitable planets or not.

Much progress has recently been made concerning the basic steps of planet formation as well as the basic astrophysical and geochemical timescale constraints. Nearer the star, the "core accretion" mechanism (Pollack et al., 1996; Ida and Lin, 2004; Alibert et al., 2005) involves collisional growth from planetesimals up to planet-sized objects. Farther away from the star, the "gravitational instability" mechanism (Safronov, 1969; Goldreich and Ward, 1973) involves "clumps" that arise from disc fragmentation. Recent advances in modeling capabilities have revealed the progression of different dynamical evolutionary stages that led to the present Solar System architecture: planet migration, followed by large-scale transport and redistribution of material, giant collisions, such as the Moon-forming event, and subsequent intense bombardment episodes (Tsiganis et al., 2005). At the same time, a deeper understanding of the post-formation geological, geochemical, and atmospheric evolution of planets and their satellites has been achieved.

On the other hand, the effects of mutual collisions on the chemical evolution of the forming planetary embryos, the relative contribution of bodies originating in different regions of the Solar System, and the relative significance of the successive formation stages in the final water budget of Earth are not well constrained. Understanding the sequence of events for Earth and other planetary bodies in our solar system will enable us to probe also the habitability of exoplanets.

\subsection{Key objectives}

Key Objective 1. To assess the elemental and chemical picture of protoplanetary stellar discs. An important aspect in planet formation theories is the evolution of protoplanetary discs in relation to their host stars. So far we have very limited data on protoplanetary discs. Observations are still scarce, but prospective Atacama Large Millimeter/ submillimeter Array (ALMA) observations should extend our knowledge and help compile more information about the distribution of different chemical species in discs and other parameters that are necessary for refining numerical models of protoplanetary disc evolution and early phases of planet formation. Hydrodynamic codes that are generally used to model such processes only allow simulations over comparatively short timescales, as the equations involved are quite complex. Hence, an improvement of existing computing capabilities is needed.

Sub-objective 1. To understand the metallicity of stars. The chemical composition of planetary systems is linked to the elementary composition of the star around which they form. Therefore the "metallicity" of stars is a crucial factor in the development of the "Solar System" and has been a major project in the Kepler study. In turn, metallicity is linked to stellar synthesis, which is a topic in its own right.

Sub-objective 2. To improve chemical models of protoplanetary disc formation and evolution. Models should take into account complex environmental (variable luminosity of the young star, stellar environment) and internal (radiative transfer, magnetohydrodynamic instabilities and turbulence, viscosity variations, etc.) processes in an effort to better constrain key parameters (e.g., solids-to-gas ratio, snowline position) that are important for deriving the correct formation timescale, size distribution, and composition of the first planetesimals, as functions of heliocentric distance.

Sub-objective 3. To improve our understanding of the evolution of circumstellar discs, in relation to their host stars. Long-term evolution of a protoplanetary disc can only be modeled by implicit hydrodynamic codes that include radiative transfer, where the physical properties are resolved to the desired level. Incorporating chemical evolution inside the disc is a necessary but difficult task-commonly used numerical codes do not include this aspect. Coupling the dynamics with the chemical evolution of the disc will constitute a great improvement.

Sub-objective 4. To determine the chemical history of key molecules (such as water, oxygen) in the evolution from molecular clouds to star-planet(s) system. Some key elements can help us reconstruct the chemical history of the evolution that, starting from the original molecular nebula, ends in the formation of the star-planet(s) system. One of the most important compounds is the water molecule. The presence of water in liquid state on a planet's surface is generally accepted as essential for its potential habitability (Kasting et al., 1993). Water in gaseous form acts as a coolant that allows interstellar gas clouds to collapse to form stars, while water ice facilitates the adhesion of small dust particles that eventually grow to planetesimals and planets. The development of life requires liquid water, and even the most primitive cellular life on Earth consists primarily of water. Water assists many chemical reactions that lead to complexity by acting as an effective solvent. It shapes the geology and climate on rocky planets, helps maintain plate tectonics, and is a major or primary constituent of the solid bodies of the outer Solar System. Thanks to a number of recent space missions, culminating with the Herschel Space Observatory, an enormous step forward has been made in our understanding of where water is formed in space, 
what its abundance is in various physical environments, and how it is transported from collapsing clouds to forming planetary systems. At the same time, new results are emerging on the water content of bodies in our own solar system and in the atmospheres of known exoplanets.

Another particularly useful investigative tool is the study of deuterium fractionation, namely, the process that enriches the amount of deuterium with respect to hydrogen in molecules. Deuterium fractionation initiates at the very early stages of the evolution of the protosolar nebula. Therefore, analyzing the $\mathrm{D} / \mathrm{H}$ ratio in different objects, which will eventually form new stars, and in comets, meteorites, and small bodies of the Solar System will provide insight into the very first steps of the Solar System's formation.

Sub-objective 5. To interconnect chemistry with disc hydrodynamics and structure. When studying the protoplanetary disc, a static disc-model is used; this is mainly concerned with the mass and the structure of the disc. One significant challenge has already arisen from observations by ALMA of discs around young stars. These have revealed distinct asymmetries in the dust continuum emission, which has led to the development of new models to explain the observations (Flock et al., 2015). Moreover, ALMA detected features that are most probably the result of young protoplanetary bodies forming in the disc around the young Sun-like star HL Tau, an unexpected observation at this stage of stellar system formation (ALMA, 2014).

Key Objective 2. To better understand our solar system: planet formation, dynamical evolution, and water/organics delivery to Earth and to the other planets/satellites. Addressing this second key objective should allow us to reach a robust theory of Solar System formation (planet formation and dynamical evolution of the early system), characterized by a well-defined sequence of all major system-changing events and calibrated with available data (e.g., geochemical) where possible. The generic mechanisms pertaining to planet formation and dynamical evolution have to be defined and studied in a wider context, while key conditions and parameters that lead to the "particular solution" of our solar system have to be identified. Our knowledge of Earth-specific events (e.g., Moon formation, a possible late heavy bombardment) has to be refined and placed in the overall context of Solar System formation. Such a theory would constitute the basic "input" for testing different astrobiological scenarios for Earth and other, possibly habitable environments in the Solar System and beyond.

Sub-objective 1. To better understand the transition from planetesimals to planets and satellites (end to end). Highperformance computing resources with increased-resolution simulations are required to address this task, taking into account the evolving disc environment and more refined collisional models (size distribution, accretive vs. nonaccretive collisions). The goal is to resolve important issues, such as understanding the short accretion timescale for the cores of the giant planets (which should be smaller than the lifetime of the gas disc) and better matching the formation timescales for the terrestrial planets and the Moon with geochemical data. At present, no numerical code of planet formation is able to perform fully self-consistent simula- tions, starting with micron-sized solid grains (i.e., chondrule-like) and ending up with fully formed planets. Hence, the development of necessary technologies to address this issue has to be supported.

Sub-objective 2. To better understand the dynamical evolution of the "young" Solar System. Emphasis should be placed on resolving ambiguities related mainly to deciding between different scenarios proposed up to now for the gasand planetesimal-driven migration of the giant planets. This has important repercussions for understanding the largescale transport of asteroidal and cometary material throughout the Solar System, the evolution of the smallbody reservoirs, the accretion of the terrestrial planets, and the sequence of bombardment episodes on the geologically evolving planets and satellites at various epochs.

Collisions between objects of different sizes (geologically differentiated or not) are an integral part of the formation process, both during the planetary accretion phase and after. Material that is essential for the development of prebiotic chemistry (water, organics) can be delivered to forming (or already formed) planets by so-called "catastrophic" events.

Sub-objective 3. To improve models on conditions for survival and/or generation of essential molecules during impacts. Information can be obtained by simulating the coupled thermodynamic and chemical evolution of the hot plasmas generated during high-velocity impacts between various types of bodies and by testing with gun impact simulation facilities (e.g., Fraunhofer Institute for High-Speed Dynamics, Ernst Mach Institute, EMI, Freiburg, Germany; and the two-stage light gas gun at the University of Kent, UK).

Sub-objective 4. To identify dynamical processes that can redistribute essential material throughout a system. The conditions under which generic dynamical mechanisms enhanced the transport of essential material throughout the Solar System should be identified. This information is also important for other planetary systems (see Key Objective 3), which may contain various types of planets in various orbital architectures.

Sub-objective 5. To better understand the effects of postformation bombardment episodes on Earth and other planetary bodies generally assumed to have been important for the development of life. Questions about whether such events bring necessary "fresh" material (water/organics), supply "energy" (heat, radiation), create "friendly" habitats (site resurfacing, exposure of subsurface material) and whether these are critical or not for the development of life have to be properly addressed (see also Research Topics 2 and 3).

To thoroughly test theoretical models, we need to have better knowledge of the distribution and physical properties of Solar System objects; for example, the outer Solar System, whose structure may provide important constraints for dynamical evolution models, is largely unexplored. More ground- and space-based observations are needed (including occultation surveys). We also need to improve our knowledge of the composition of primordial objects; on a decadelong plan, this means enhancing our efforts to collect and analyze a wide variety of meteorite samples (including 
extending fireball observations networks), the only low-cost way of collecting primordial material. Finally, important constraints for impact modeling could be derived by further enhancing laboratory research (impact experiments).

Sub-objective 6. To better define the timeline of the formation of the Solar System and water/organic delivery on Earth. In all the scenarios proposed for the early evolution of the Solar System (i.e., the first $\sim 10$ million years), the presence of water and volatile elements inside the water-ice condensation line appears to be a natural by-product of the appearance of the giant planets. It is presently unclear, however, how much of these volatile elements would actually survive the formation process of the terrestrial planets. The same holds true for the organic material originally present in the ice-rich planetesimals: did these organics, that were incorporated in the growing terrestrial planets, survive the impacts, or were they destroyed?

Sub-objective 7. To interpret the temporal link between Solar System evolution and the rise of life on Earth. Earth and the Moon completed their formation about 4.5 billion years ago (i.e., about 30-100 million years after the formation of the Sun and the first solids, calcium-aluminumrich inclusions, in the Solar System), yet the oldest proven evidence for life on Earth is no older than $3.5 \mathrm{Ga}$, although it is likely that life had already appeared before this time. Further, due to recycling of the crust, older, well-preserved rocks have not survived tectonic recycling of the crust. Although life most likely appeared well before the event known as the Lunar Cataclysm or the proposed Late Heavy Bombardment (LHB), which should have ended about 3.8 billion years ago (Westall, 2012), this event must certainly have had an important impact on Earth, probably contributing to the destruction of the crust and erasure of much information about the oldest events on our planet. The exact nature of this correlation is, however, uncertain. Did the LHB play a role in resupplying the terrestrial planets with organic material and water that possibly got lost during their formation process? Did the LHB accelerate chemical evolution by providing, for example, surface restructuring and/ or energy on small (local) scales? Or was the LHB simply the final event in the long-lasting, violent process of terrestrial planet formation that did not contribute directly to the appearance of life on Earth but only marked the onset of a more quiescent era, characterized by a life-friendly environment? (See also Fig. 1 and Research Topics 2 and 3.)

Key Objective 3. To better understand the diversity of exoplanetary systems and the development of habitable environments. Detailed information from observed stardisc configurations will enable us to constrain a general planet formation theory, which is the main task to be addressed. Different properties of discs (solid-to-gas ratio, density and viscosity profile), host stars (luminosity, spectral type, metallicity, etc.), and their environment (gravitational torques and radiation from neighboring stars) should be considered.

Sub-objective 1. To better understand the dynamical mechanisms that lead to the observed diversity of exoplanetary architecture, and assess how they affect habitability.
Various dynamical mechanisms have been proposed to explain the observed exoplanetary architecture. This subobjective's goal is to identify the key generic mechanisms responsible for the onset of dynamical instabilities during different stages of formation, and their relationship to fundamental characteristics of the initial system. Orbital dynamics are frequently used as an indicator of the habitability of a planet but are not sufficient (see Section 1.3.2). This should enable us to constrain dynamical evolutionary pathways (including water delivery and bombardment episodes) and final dynamical configurations of planetary systems that are compatible with the development of prebiotic chemistry and, possibly, life.

Sub-objective 2. To identify biomarkers and promising methods of detection. Observations of atmospheres of different types of planets (Earth-like, warm and hot superEarths, "hot" Neptunes, etc.) are crucial for understanding their postformation evolution in relation to different parent stars. For planets labeled as "habitable," spectrally resolving their atmosphere is currently the only foreseeable means of detecting possible signatures of life. However, a theoretical effort will have to be executed to decide which characteristics constitute real "biomarkers," to properly model them (see Research Topic 5), and to discern what new technologies will need to be developed so as to observe them. This work will constitute an important asset for designing future missions.

Sub-objective 3. To find out how the study of exoplanets can help fill the gaps in our understanding of the formation of our own solar system. Observations by Spitzer (NASA) and Herschel (ESA) suggest that debris discs are at least as common around nearby stars as planets. A study of the nearest G-type stars by Wyatt et al. (2012) indicates a correlation between low-mass planets and observed debris discs, concluding that systems with only low-mass planets are preferentially dusty. High-resolution observations as provided by ALMA are needed to understand this early phase in the formation process, which defines the initial chemical distribution for the material of which terrestrial planets are formed. With observations of different ages of a disc, we could improve the existing models also for the Solar System.

\subsection{Approach to achieve the key objectives}

Next ten years. Observations. More and better-quality observations from ground and space are needed. The last generation of millimeter and submillimeter interferometers (ALMA and the Northern Extended Millimetre Array, NOEMA) has the required sensitivity and spatial resolution to provide new and strong observational constraints on chemical abundance of molecular species to chemical models for all the phenomena that play a key role in planetary system formation, namely, prestellar cores, protostars, young stellar objects, and protoplanetary discs. Because of these two important observing facilities, major advancements are expected in the following:

- Observations and detection of complex molecules;

- 3-D disc models including chemistry;

- Combination of hydrodynamics and chemistry;

- Comparison with astrochemistry laboratory experiments. 
Protostellar nebular (PSN) observations made by ALMA and the upcoming James Webb Space Telescope (JWST) are needed to obtain necessary information on early stage of formation (of planetesimals, embryos, and protoplanets) from which new models can be developed to bridge the gaps in our understanding of the formation process.

However, because of the high abundance of water in Earth's atmosphere that can partially obscure the observations from ground-based facilities, the bulk of the data will come from space observatories. Except for in situ mass spectroscopy in planetary and cometary atmospheres, all information about interstellar and Solar System water comes from spectroscopic data obtained with IR telescopes [the Infrared Space Observatory (ISO), Herschel]. The validation of chemical models with the Herschel observations is ongoing work.

An improvement of observational and technical facilities should also be pursued. The European Extremely Large Telescope (E-ELT) and PLATO 2.0 will open a new era for the European astronomical community. The results from various observational campaigns will offer increased added value for the theory, if collaboration between observers and theorists is optimized in the framework of a larger network and follow-up programs supported by the European Union.

Modeling. Our ability to develop sophisticated theoretical models depends critically upon our knowledge of the relevant physical and chemical processes, as well as on our computational capabilities. In particular, the early stages of planet formation are still not well understood. The developed models for the growth of planetesimals from dust grains by subsequent sticking collisions have revealed obstacles to dust growth, such as bouncing, fragmentation, and radial drift barriers (referred to as the "meter-size barrier").

Such knowledge gaps on specific key points prevent us from formulating a robust theory; there are, however, several solutions that have been proposed to overcome these gaps. Therefore, a robust, system-independent, theoretical framework has to be developed, which could successfully be applied to explain the diversity of observed (and others that cannot yet be observed) planetary systems and place our singular life-bearing example-the Solar System-in context. Improving our theoretical tool kit should be based on supporting basic research in planetary science and further promoting collaboration on a European scale, by further developing interdisciplinary networks. However, special provisions have to be made in future research programs to exploit and safeguard important expertise (currently dispersed in, for example, small groups, working in small institutes and/or in less-favored regions in Europe).

At present, testing theoretical hypotheses is heavily based on accurate, high-resolution computer simulations. Our current capabilities, however, do not suffice. Hence, research groups and institutes should be supported so as to invest in new technologies (hardware, parallel computing protocols) and key scientific software development.

The refinement of theoretical models is based also on the availability of data from space missions. For example, results from impact experiments need to be calibrated on large scales, which are inaccessible in laboratories. Hence, an impact-experiment mission like Asteroid Impact and Deflection Assessment (AIDA, a joint ESA/NASA mission) should produce extremely important results. We note that the European part of AIDA [called Asteroid Impact Mission (AIM)] is currently in Phase A/B1 study by ESA.

We expect that a robust theory of formation for our solar system can be developed within the next decade, or at least the main uncertainties will be sufficiently studied, to significantly narrow the admissible set of solutions. In particular, we expect significant progress towards resolving the main challenges related to the following:

- Formation of planetesimals and planet cores,

- Dynamical evolution scenarios,

- Impact history of early Earth.

This would provide a well-constrained time line of the main events that sets the picture for the development of life on Earth.

Furthermore, it is a central aim during the next decade to improve our theories to the point where the key parameters that affect the observed diversity of planets and their orbital architecture will be elucidated. This will enhance the theoretical background for PLATO 2.0, which is expected to bring Europe into a new era in the field of exoplanetary science. Also, we expect to have achieved a better understanding of habitable conditions and the associated biomarkers that we should be looking for in the atmospheres of habitable planets.

Interdisciplinary collaboration. Progressing on this research topic requires input from many different areas of expertise; interdisciplinary research groups have to be linked together in order to tackle the various key scientific objectives adequately. To develop knowledge of exoplanets and habitability in particular, it is important to create and strengthen interactions and collaborations with the nuclear community that explores stellar synthesis. Such collaborations were apparent in European Science Foundation programs GREAT (Gaia Research for European Astronomy Training), EuroGENESIS (Origin of the Elements and Nuclear History of the Universe), CompStar (the New Physics of Compact Stars), and COSLAB (Cosmology in the Laboratory).

Ten to twenty years from now. Advanced physicochemical models. The role of magnetic fields, the generation of turbulence by fluid instabilities, and their relation to the distribution of different chemical species are of profound importance for understanding the condensation sequence; the evolution of the density and temperature profile; the formation of the first solids; and the dynamical evolution of grains, pebbles, and planetesimals in protoplanetary discs. This is the most fundamental step in planet formation. Hence, advanced models that combine all major physical and chemical processes (gravity, magnetohydrodynamics, collisions, thermodynamics, chemistry) in a self-consistent manner should be developed. Statistical analyses of their range of solutions will then enable us to understand how the observed diversity of planetary systems is produced and how our solar system fits in. This requires advances not only in theory but also in technology (computer platforms and software).

Candidate list for habitable exoplanets. Building on the results of the next decade, we should be able to develop a system-independent theory that will enable us to understand 
the details of planet formation around different stellar classes (from $\mathrm{F}$ to $\mathrm{M}$ ). We will have considerably expanded our database of Earth-like planets in habitable zones around different stellar classes. Improvements and new awareness due to PLATO 2.0 and E-ELT observations should enable us to compile a candidate list for habitable exoplanets.

Solar System missions. Theoretical models should be further refined that will enable us to understand more of the fine details of Solar System formation. This, however, requires additional data from space missions, such as dedicated sample return missions from small bodies (primitive-type asteroids, comets, main-belt transition objects) that will allow us to access samples of primordial material. Sample return missions from Mars will, of course, be of utmost importance for astrobiology. Also, missions to satellites of the outer planets, which have been identified as additional potential candidates for hosting life, should be pursued (e.g., Europa, Titan). The development of the required observational technology to achieve these objectives needs to be promoted.

Sample return missions would greatly benefit from the development of a European extraterrestrial sample-receiving and curation facility, the requirements of which are presently compiled and defined in the European Curation of Astromaterials Returned from the Exploration of Space
(EURO-CARES) project (supported by the European Union Horizon 2020 program). At the same time, sample return missions raise planetary protection issues that need to be properly addressed.

After twenty years. Solar System missions. Solar System exploration will enable an increasing quantity of pristine material to be accessed, which will allow, in parallel with improved computing and analytical capacity, additional insight into the formation of planetary systems. Figure 3 gives an integrated view of Research Topic 1 together with its timeline.

\subsection{European strengths and needs}

Europe has been a leader in numerous fields of exoplanetary research, and many world-renowned experts on observations and theory of planetary science work for European institutes, performing high-quality research. Different countries in Europe (such as Germany, Austria, and Switzerland) have already established national and transnational networking groups on this research topic, and these actions should be further supported.

Upcoming European space missions like CHEOPS and PLATO 2.0 as well as new ground-based telescopes (e.g., E-ELT) will certainly improve the quality of observations and provide exciting new data. Europe has ongoing programs

\section{Research Topic 1 - Origin and Evolution of Planetary Systems}

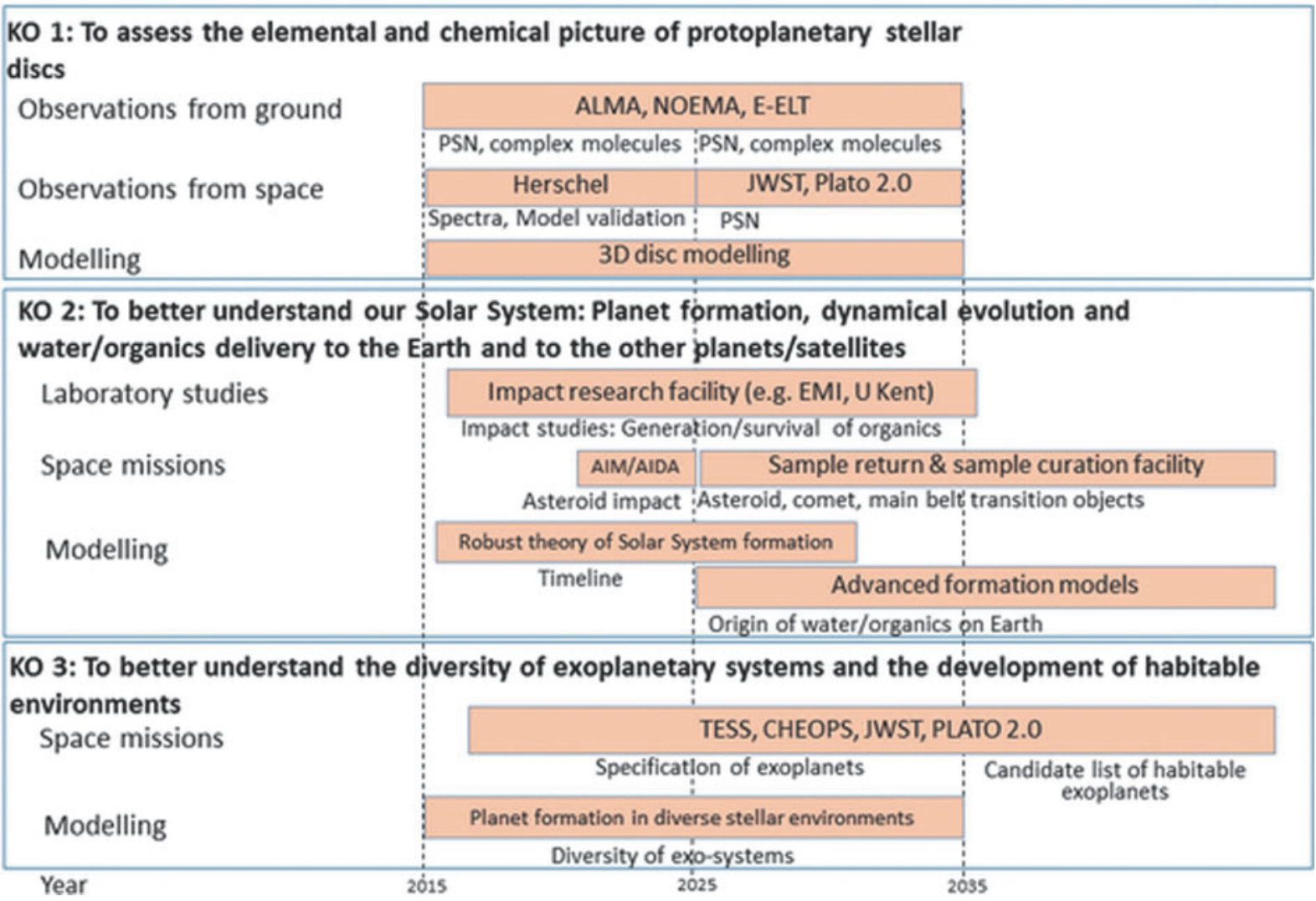

FIG. 3. AstRoMap Roadmap, approaches to reach the key objectives of Research Topic 1 "Origin and Evolution of Planetary Systems"' within the next 10, 20, or follow-on years. 
(e.g., CARMENES: Calar Alto high-Resolution search for M dwarfs with Exoearths with Near-infrared and optical Échelle Spectrographs) in place to study planets orbiting cooler (M dwarf) stars, which are considered favorable targets for finding habitable, Earth-like planets (see Research Topic 5).

European astronomers have taken a leading role in the development of dynamical models to simulate the evolution of planetary systems, for example, the Nice model for the Solar System (Tsiganis et al., 2005). For the European planetary science community, the European Planetary Network Europlanet was founded in 2005 with the aim of acting as a platform for the exchange of ideas, personnel, tools, and data (see http://www.europlanet-eu.org). Meanwhile, more than 60 institutions are members of Europlanet. In addition, in recent years many European countries (e.g., Germany, Switzerland, Austria, Belgium, France) have launched national networking programs on planetary science, which have increased the capacity of front-line European teams by promoting collaboration between scientists of different backgrounds (geology, astrophysics, celestial mechanics). Strong observational capabilities exist (e.g., European Southern Observatory, ESO), and significant experience has been gained by European teams in designing, executing, and exploiting data from space missions, within the framework of collaboration between national space agencies and ESA. An example is the ESA space mission Rosetta that allows for the study of the dynamics of a Jupiter-family comet (Altwegg et al., 2015).

However, European missions to outer Solar System bodies are significantly limited by technological challenges, such as energy (unavailability of radioisotope thermoelectric generators) and data transmission rate. Also, the scientific return from such missions could be greatly enhanced if provisions were made to perform flybys of targets of opportunity in the asteroid belt during cruise phase. European and national space agencies should consider such options, in relation with the scientific community, whenever opportunities arise.

As for the various other astrobiology research activities (see Section 8.2), European investigation of the origin and evolution of planetary systems needs to be better coordinated, for example, within a large scientific network (or Virtual Institute). Such networking activities would promote interactions of groups from different research fields, an important necessity for this interdisciplinary research topic. A first step in this direction has already been achieved by the networking activities of Europlanet, COST ORIGINS, and EANA.

\section{Research Topic 2: Origins of Organic Compounds in Space}

\subsection{State of the art}

Almost 200 organic molecules have been detected as gaseous species in space (http://astrochymist.org/ astrochymist_mole.html). They are formed in cold molecular clouds and in the early phases of star formation. Gasphase chemistry cannot explain the abundances observed for most of them. It is therefore thought that most of them form on cold dust grains on which atoms and molecules accrete to form icy mantles. As demonstrated by a large number of laboratory studies (e.g., Allodi et al., 2013), surface reactions and/or energetic processing (by UV photons, electrons, atoms, and ions) induce the formation of a solid complex organic refractory material (often referred to as "organic residue") and of a plethora of molecules that, once released by thermal (e.g., around a forming star) or nonthermal (e.g., by sputtering in shocks) processes, are observed in the gas phase. Circumstellar dust grains are the building material of planetesimals and, in turn, of larger planetary objects (see Research Topic 1). Thus, there is a link between the composition of preplanetary materials and that of planetesimals, comets being assumed to be their direct remnants. This scenario has been recently confirmed and extended to protoplanetary discs by Öberg et al. (2015). Those authors showed that complex organics accompany simpler volatiles in protoplanetary discs, and that the rich organic chemistry of our solar nebula is not unique. It is also widely accepted that comets and other small bodies, such as asteroids, meteorites, and interplanetary dust particles, have delivered huge amounts of molecules (including water and organics) to Earth (and possibly on exoplanets), especially during its early history when the flux of extraterrestrial materials was much higher (e.g., Schmitt-Kopplin et al., 2010; Westall, 2012). This scenario demonstrates the evident role of me- teorites in this field. Evidence for catalytic properties of meteorites regarding the synthesis of organics was recently provided by investigating thermal processes of widely diffused chemical precursors (e.g., formamide, $\mathrm{HCN}$ ) at relatively high temperatures (333 and $413 \mathrm{~K}$ ) (Saladino et al., 2013) or at the most extreme temperature conditions during impacts on Earth's atmosphere or surface (Ferus et al., 2014). Structurally complex nucleosides (as well as a variety of other biologically relevant molecules) have been synthesized from formamide by proton irradiation at 170 $\mathrm{MeV}$, mimicking the effect of the solar wind (Saladino et al., 2015).

These results have been obtained by observational, experimental, and theoretical studies. The advent of a new generation of telescopes, including the interferometer ALMA, the Square Kilometer Array (SKA), and JWST, will enable scientists to study the distribution of abiotic organic molecules in star-forming regions to a much greater extent and so learn more about the relationship of these molecules to exogenous delivery on Earth or potentially habitable exoplanets.

\subsection{Key objectives}

Key Objective 1. To promote our understanding of the diversity and the complexity of abiotic organics. The interaction between electromagnetic radiation and organic molecules in heterogeneous environments is a prebiotically relevant process. Minerals may have played a pivotal role in the prebiotic evolution of complex chemical systems, mediating the effects of electromagnetic radiation, influencing the photostability of organic molecules, catalyzing important chemical reactions, and/or protecting molecules against degradation. In particular, nucleobases are relevant biomolecules to investigate in the prebiotic context, because 
they code components of nucleic acids, and from the standpoint of the survival of biological systems in space conditions (Fornaro et al., 2013; Vergne et al., 2015). Several studies on the photodynamics of nucleobases suggest that their structure could have been naturally selected for their ability to dissipate electronic energy through ultrafast photophysical decay.

Sub-objective 1. To study the mechanisms for the formation of organics and their evolution under space conditions. Considering the putative involvement of minerals in prebiotic chemistry, it is necessary to study the photostability of nucleobases under space conditions in the presence of mineral matrices in order to investigate both the prebiotic processes that might have had a role in the development of the first living entities on Earth and the physical and chemical processes occurring in extraterrestrial environments. As mentioned above, thermal equilibrium chemistry, surface chemistry, and energetic processing of interstellar dust produce several complex organic molecules observed in space and eventually delivered to Earth. It is not yet clear how complex these molecules are. Molecular abundance decreases with increasing molecular complexity, and it becomes virtually impossible to detect them in either laboratory experiments or in space by astronomical observations. Of course, the limit of observability changes as the detection techniques become more sensitive.

Sub-objective 2. To better understand the role of catalysts in the formation processes of organics. An additional issue that is not fully explored is the role of specific solid substrates, such as catalysts (see also Research Topic 3). As an example, it is very important to increase knowledge of the reactivity and the catalytic role of meteorites (and cosmic dust particles that are collected on Earth and/or in space) in the synthesis of organic molecules of high structural complexity (possibly intermediates of both the genetic and metabolic mechanisms). For example, meteorites of different origin and composition, available from international scientific collections, can be studied and compared for their reactivity under different flux and energy conditions ( $\mathrm{keV}, \mathrm{MeV}$ ions or UV photons) using the most simple and widely diffused chemical precursors (e.g., one-carbon-containing compounds such as formamide, $\mathrm{HCN}$, isocyanate, and others). Different temperature conditions should be tested depending on the nature of the energy source applied. In a similar way, cosmic dust analogues that are prepared in the laboratory by different deposition procedures with a specific and controlled elemental composition can be evaluated for their catalytic properties. Data about yields, complexity, and (eventual) selectivity should be correlated to elemental composition and mineralogical origin to better define the effect of metal composition.

Key Objective 2. To better understand the molecular evolution of abiotic organics present in Solar System objects, including early Earth, under the combined role of physical agents such as thermal variations, high-energy particles, photons, and solar wind irradiation. Complex organic materials exist in the protoplanetary solar nebula either in the solid state (refractory organic material) or as gaseous molecules. Such materials are completely destroyed near the young Sun but can be at least partially preserved in objects formed far from the central star (e.g., comets and some classes of asteroids). These objects and their debris (meteorites and dust particles) are in turn subjected to an extreme variety of temperature conditions, to energetic photons, and to ionizing radiation of galactic and solar origin that modifies the organic material. Ad hoc experiments and observations are necessary, aimed at reproducing different environments that are potentially relevant to astrobiology. These clearly include Mars, Titan, the icy moons of the giant planets as well as comets (both from the Oort cloud and the Kuiper belt) and some classes of asteroids and their debris (meteorites and dust particles). Within the Solar System, the surfaces of those objects are exposed to the electromagnetic solar spectrum and to ionizing radiation mostly generated by the Sun (solar wind and solar cosmic rays, SCR, primarily protons accelerated by flares and coronal mass ejections to energies typically of tens to hundreds of megaelectronvolts). Also relevant are high-energy (i.e., more penetrating) particles coming from the Galaxy (galactic cosmic rays, GCR). SCR and GCR are dominated by protons that exhibit different energy spectra (see, e.g., Bennett et al., 2013). The flux of energetic ions and electrons in the jovian magnetosphere that irradiate the icy surfaces of satellites and rings is particularly intense. Such a process induces a continuous exchange of atomic and molecular species with their environments (e.g., the tenuous atmospheres of the satellites and the gas surrounding the particles in the rings). Chemical species are continuously expelled from surfaces by exogenic processes, such as sputtering caused by the high fluxes of bombarding energetic ions and electrons from the jovian magnetosphere (e.g., Johnson et al., 2008). This is a well-studied phenomenon, and data concerning sputtering yields exist for a wide range of combinations of projectile energy and target composition. Sputtered species include mostly neutral atoms and molecules as well as ionized species (the latter include clusters and deserve further study). The released species can be lost to space or can populate the exospheres of the icy satellites (e.g., Plainaki et al., 2015). In the coming years, it will be important to collect further data on the nature of the species released by ion irradiation of realistic ice mixtures, as well as their yields and energy and angular distribution. Additional ice-gas interactions are due to endogenic geological processes, such as geyserlike activity that may produce plumes as those observed at the icy moon Europa (Roth et al., 2013). Chemical compounds present in the plumes could originate in the putative oceans underneath the surface, and once released in the atmosphere and observed by JUICE (the long-term ESA mission to the jovian system) instruments, they could give information on the (bio?)chemistry occurring in the underlying oceans.

The interaction of organic chemical precursors with the surrounding material in the form of silicates (e.g., in meteorites) or ices (e.g., on a dust aggregate expelled from a comet) modifies their excitation states, producing energetically favorable conditions for the generation of reactive radical species able to be further transformed into very complex organics (Adhikari et al., 2000). A key question is, can the interaction between energetic protons (and/or other ions) and organics on meteorites (used as template for materials coming from space) offer a benign environment for the formation of prebiotic molecules during their journey 
through space, during their impact, and during their lifetime on a planetary surface? Some experimental evidence to address this issue has already been produced. As an example, Simakov has described the possibility of prebiotic syntheses under experimental conditions that simulate the energetic processing of the mineral surface of asteroids and meteorites. In particular, mixtures of nucleosides (ribose and 2'-deoxyribose derivatives) and inorganic phosphate $\left(\mathrm{NaH}_{2} \mathrm{PO}_{4}\right)$ were exposed to space irradiation on Bio-Sputnik to yield the corresponding nucleoside derivatives (Simakov, 2008). An additional important contribution comes from the results of the EXPOSE-E and EXPOSE-R chemical experiments on prebiotic photochemistry, as it occurs in the interstellar medium or in the clouds of Saturn's moon Titan, and on the stability of organic compounds and microorganisms under simulated martian surface conditions (EXPOSE-E, 2012; EXPOSE-R, 2015). Organics in space can be delivered to all objects in the Solar System, including Earth, where they can be in contact not only with rocky materials but also with water both in the liquid and in the solid phase (e.g., early Earth, icy moons of the outer Solar System or planetesimals formed beyond the snow line). While aqueous alteration of extraterrestrial organic matter is well studied (see Research Topic 3), less information exists on the interaction promoted by energetic penetrating radiation (e.g., energetic protons) at the interface of water (liquid or frozen) and solid (rock and/or organic).

Key Objective 3. To understand the role of spontaneous inorganic (organic) self-organization processes in molecular evolution. Self-organization processes that spontaneously occur in both inorganic and organic systems can facilitate the synthesis of biomolecules that act as catalysts, protective environments, and template facilities. For example, $\pi-\pi$ interactions (HOMO-LUMO) between the aromatic moieties of purine nucleobases in cyclic nucleotide monophosphates favor, in solid or liquid phases, the spontaneous formation of pillared columns of molecules that are very organized and react to spontaneously yield oligonucleotides with the appropriate regiochemistry (which proceeds via socalled "click-like oligomerization" without a template) (Di Mauro et al., 2015). A similar process can occur in inorganic systems. For example, inorganic membranes can spontaneously grow from silica solution and traces of metal salts under experimental conditions intended to model primordial planetary conditions (including pristine Earth) (García-Ruiz, 2009). These membranes can act as catalysts in the prebiotic synthesis of biomolecules, protecting the novel products from possible degradation processes.

Sub-objective 1. To identify and structurally characterize novel spontaneous self-organized inorganic and organic systems

Sub-objective 2. To determine the mechanism of spontaneous self-organized systems and their role in the prebiotic synthesis of biomolecules

\subsection{Approach to achieve the key objectives}

Next ten years. Laboratory studies. Laboratory work simulating organic chemistry in space has already been well developed in Europe, although it has spread over a large number of relatively small facilities. It is commonly thought that many more relevant molecules are still to be revealed in laboratory experiments and possibly confirmed by astronomical observations. In fact, the detection techniques used up to now, mostly based on UV-vis-IR spectroscopy, are sensitive only to those synthesized species whose abundances are larger by a factor of several thousand compared to the astronomically observed values. At present, there are ongoing efforts in some laboratories distributed worldwide to build up and use experimental apparatus based on new techniques that are different from the traditional, widely used approaches. These techniques include novel methods (e.g., nanoscale secondary ion mass spectrometry and twostep laser ablation and ionization mass spectrometry) that are more sensitive and can better demonstrate the formation of additional (complex) molecules and/or fragments that could be of primary relevance (see, e.g., Allodi et al., 2013). With increased support of simulation work, a much more complete inventory of molecules produced by surface chemistry and energetic processing of ices should be obtained by the end of the next decade.

The interaction between ice and rocky materials with organics, present in the different environments promoted by the (synergic?) effects induced by ionizing radiation and thermal excursions, has to be investigated with particular effort placed in fully controlled experimental facilities. Of course, much data already exists, both from observations (e.g., the plethora of cometary molecules observed in cometary comae, see http://astrochymist.org/astrochymist_comet.html) and from laboratory experiments. These experiments have to be continued in a coordinated way and should be the basis of a "comparative astrobiology," the focus of which would be to better understand the similarities and specificities of the (bio)chemistry possible in the different environments. Such information would be essential to understanding whether life exists or existed in other objects in the Solar System and would contribute to our understanding of the origin of life on Earth and possibly on exoplanets.

Spontaneous organic and inorganic self-organized systems have been simulated in European laboratories. Novel systems should be designed and fully characterized. Their connection with the prebiotic synthesis of biomolecules of relevance for the origin of life needs to be studied in more detail, with particular attention to the selectivity and regionchemistry of the processes (especially in the case of polymerization reactions), as well as to catalysis phenomena. In spacelike conditions, more attention should be paid to chemical transformations that occur under high-energy particle or photon irradiations, thus providing information for better modeling of prebiotic processes in icy media or primitive planetary conditions.

Exposure experiments in space. Currently, the analysis of exposed materials in the EXPOSE experiments on board the ISS is performed exclusively on the ground after retrieval of the samples. It would be a relevant step forward for the next 10 years if at least some analysis could be performed in situ, that is, directly on board space platforms. A first step in this direction was achieved during the Organism/Organic Exposure to Orbital Stresses (O/OREOS) mission where UV/ vis/near-IR transmission spectra were retrieved by telemetry from the experiments on board an Earth-orbiting nanosatellite (Cook et al., 2014). 
Theoretical studies. The already well-developed theoretical studies on organic chemistry in space should be further elaborated, as, for example, those investigating the energy profile of excitation states for some of the main chemical precursors in the synthesis of organics (e.g., formamide, $\mathrm{HCN}$, isocyanate, and others) under high-energy conditions and estimations on the effect due to surface interaction and metal complexation processes.

Theoretical studies on spontaneous inorganic (organic) self-organization processes are also required to fully characterize the structure of supramolecular aggregates, as well as to understand possible relationships between inorganic (organic) self-organization systems and the emergence of complex biomolecules (peptides, oligonucleotides, polysaccharides).

Ten to twenty years from now. Exposure experiments in space. It would be beneficial to have permanent facilities that allow direct exposure (and in situ analysis) of materials to space environments. This could be done in an ad hoc laboratory in a space station and/or on the Moon.

Specific space experiments should be designed in which the self-organization process is connected to self-catalysis for the synthesis of biomolecules under different energy conditions.

Experiments mimicking conditions in space and on terrestrial planets. It is essential to develop an understanding of the transformations that prebiotic molecules may undergo over their real time spans and in their real environments by performing laboratory experiments under conditions that mimic space and terrestrial planet environments. The interaction between the dynamic environment on early Earth (or other planetary surfaces) and prebiotic monomers might have played a prime role regarding the appearance of self-sustaining and replicating entities (see Research Topic 3). Hence, the quantification of processes occurring in space can provide additional insights into the organic inventory of a planet.

Curation facility for samples returned to Earth. It would be relevant to have a fully operative receiving, curation, and analysis facility (for meteorites, interplanetary dust particles, and returned samples from space missions) that could be combined with the most sophisticated experimental techniques in a European Laboratory for Astrobiology (see Research Topics 1 and 5). At present, there are several small-to-mediumsized European laboratories that are doing an excellent job. In the future, however, it will be necessary to have larger facilities available with breakthrough instrumentation.

Interdisciplinary collaboration. Projects should be implemented that allow scientific crossover of information between the different science communities (chemistry, geology, astrophysics, biology, etc.).

After twenty years. Fully equipped and functioning laboratories on Earth and possibly on the Moon, along with results

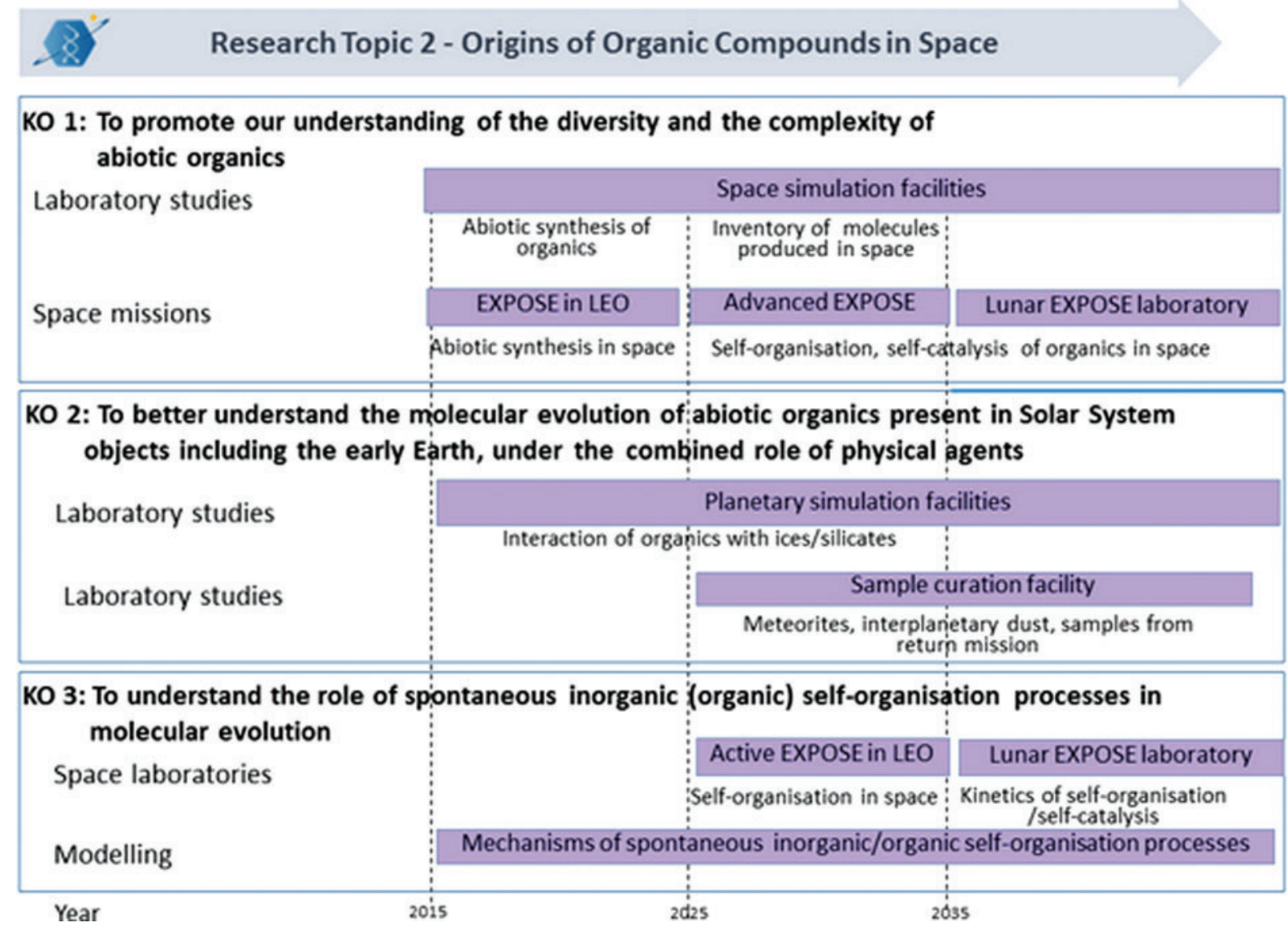

FIG. 4. AstRoMap Roadmap, approaches to reach the key objectives of Research Topic 2 "Origins of Organic Compounds in Space" within the next 10, 20, or follow-on years. 
from astronomical observations, would enable a more complete picture of abiotic evolution. We should have the capacity to start from simple molecules and synthesize, in fully controlled experiments, their evolution towards those molecules that are at the interface between abiotic and biotic evolution.

Life affects its environment, and at the same time the environment affects life. This give-and-take is often expressed in feedbacks within planetary systems, that is, responses to change that either resist or enhance the perturbation and tend to stabilize an environment at a particular state, transfer it to different stable states, or send it into a runaway state. The nature, extent, and prevailing directions of these feedbacks are poorly understood. We should pursue more informed answers to questions regarding the existence of life in other environments in the Solar System and better understand the role that organics from space play in the origin of life on Earth (see also Research Topic 3). Figure 4 gives an integrated view of Research Topic 2 together with its timeline.

\subsection{European strengths and needs}

Europe plays a leading role in many relevant subfields. This is due to the coordinated efforts of many groups across
Europe. As an example, it is relevant to outline the role played by the European COST Actions that have been, and are, very important in promoting collaborations between European groups with different backgrounds. Presently, there are two COST Actions particularly relevant in the field: Astro-Chemical History (CM1401; chair: Laurent Wiesenfeld) and Origins and evolution of life on Earth and in the Universe (ORIGINS) (TD1308; chair: Muriel Gargaud).

Recently, some of the Europlanet network members have been successfully involved in the European Union Horizon 2020 program, in particular through the EURO-CARES project, which was launched in January 2015 and has as its primary objective the definition of a sample curation facility to allow the analysis and preservation of extraterrestrial materials.

Also relevant is the role that Europe has in experiments undertaken by exposing organic and biological material to solar electromagnetic and particle radiation. Experiments have been performed on board Mir, EURECA, Biopan, EXPOSE-E, EXPOSE-R, and presently EXPOSE-R2 on the ISS (e.g., Rabbow et al., 2015), in addition to ground-based research with heavy ion accelerators.

\section{Research Topic 3: Rock-Water-Carbon Interactions, Organic Synthesis on Earth, and Steps to Life}

\subsection{State of the art}

Geology and geochemistry provide the boundary conditions for our understanding of life on Earth - the best-studied planet so far and an example for more distant systems. Central to this understanding are rock-water-carbon interactions between carbon and the environment, which have been too long neglected and/or unrecognized by relevant neighboring disciplines.

Rock-water-carbon interactions dissipate energy at a planetary level. At a microscopic level, the energy produced by these interactions is expended by microbial metabolisms. Thus, the "rocks" in microbial cells are represented by catalytic Fe-Ni-S clusters in enzymes of the core carbon and energy metabolism (Russell and Hall, 1997; Wächtershäuser, 2006). On the planetary scale, the main process of energy dissipation on metal-rich planets such as Earth is serpentinization. During serpentinization, water circulates through hydrothermal systems and chemically reacts with rocks (Shock et al., 1998). Electrons from the inexhaustible reservoirs of reduced iron in Earth's crust are transferred to water, generating $\mathrm{H}_{2}$ (Holm et al., 2015) and, to $\mathrm{CO}_{2}$, generating methane and other reduced carbon compounds (Schrenk et al., 2013; McDermott et al., 2015). Methane in fluid inclusions in plutonic rocks could also be released by circulating hydrothermal fluids (McDermott et al., 2015). These products of serpentinization become dissolved in the hydrothermal fluids and are discharged at vents into the ambient seawater (McCollom and Seewald, 2013). The significance and ubiquity of rock-carbon-water interactions represent a paradigm-changing concept that has already had tangible impact on microbiological aspects of life's origin, including the most widely read undergraduate textbooks
(Madigan et al., 2014). Geologists and biologists have begun to work together on this topic to provide new, very explicit and testable theories for life's origin in a realistic geological context on Earth and on other planets (Martin et al., 2008; Arndt and Nisbet, 2012; Stüeken et al., 2013; Westall et al., 2013; Baross and Martin, 2015).

The main ingredients of these essential planetary chemical reactions are carbon of extraterrestrial (see Research Topic 2) or terrestrial (atmospheric, crustal) origin or from $\mathrm{CO}_{2}$, liquid water, transition metals (e.g., $\mathrm{Fe}, \mathrm{Ni}, \mathrm{Mo}$ ) and, in essential supporting roles but probably not as participants in the core exergonic reaction, nitrogen and sulfur. Central to microbial activities are energy-releasing (exergonic) redox reactions that are coupled to carbon chemistry, which generates the substance of cells (Fuchs, 2011; Schuchmann and Müller, 2014). Energy conserved from the core exergonic reaction also powers monomer polymerization and growth. Of all naturally occurring geochemical reactions currently known, only the process of serpentinization involves exergonic redox reactions that emulate the core bioenergetic reactions of some modern microbial cells (Buckel and Thauer, 2013). Moreover, organisms that use such primordial reactions to harness carbon and energy inhabit the rocky interior of our planet today (Chapelle et al., 2002; Lever et al., 2010) and formed an important part of microbial biomass on primitive Earth (Westall et al., 2015a, 2015b).

\subsection{Key objectives}

Key Objective 1. To better characterize and understand the dynamic redox interactions of rock, water, and carbon in their geological context on planets and moons. On 
wet rocky planets and moons, planetary thermodynamics requires that heat generated from within the planet has to be radiated to space. This generates convective currents in which surface water is circulated through the upper crust. During the early part of planet formation, the heat flux from the mantle is high, gradually decaying as the core and mantle cool. For instance, the temperature of the mantle on early Earth was $\geq 300^{\circ} \mathrm{C}$ higher than today (Herzberg et al., 2007), with consequent effects on hydrothermal and geochemical circulation (Arndt, 1994). Thus, understanding the internal structure of planets and their dynamics and evolution is an important component of addressing the flux of energy available from the mantle (as well as from natural radiogenic decay) over the lifetime of the target body. The mere possibility that life might have had chemolithoautotrophic origins on Earth also impacts our view of habitable zones. For example, the recent discovery of hydrothermal activity and evidence for serpentinization processes on Saturn's moon Enceladus (Hsu et al., 2015) demonstrates that the chemical prerequisites for rock-water-carbon interactions that are far from equilibrium can exist without energy input from solar radiation.

During the process of serpentinization, water circulating through Earth's crust reacts with iron- and nickel-containing minerals at depths on the order of a few kilometers. In that process, electrons change hands, leaving their native iron and nickel source, being transferred to water to generate $\mathrm{H}_{2}$, a powerful source of energy and mobile, accessible electrons. Carbon dissolved in the circulating water reacts in much the same manner to generate reduced carbon compounds. Serpentinization is the main energy-releasing reaction on young planets; it is similar to-and possibly the precursor of - the core energy-releasing reactions that are essential to microbial life. The contribution of serpentinization to organic synthesis on Earth is newly recognized (Proskurowski et al., 2008). This process clearly needs to be more intensely investigated and better understood.

Key Objective 2. To better characterize and understand transition metals as electron sources and catalysts in geoorganic chemistry. The chemistry of the serpentinization reaction of magnesium-iron silicate and water to serpentinite and magnetite and hydrogen was summarized by Bach et al. (2006) as follows:

$$
\begin{aligned}
& 2 \mathrm{Mg}_{1.8} \mathrm{Fe}_{0.2} \mathrm{SiO}_{4}+3 \mathrm{H}_{2} \mathrm{O} \rightarrow \\
& \mathrm{Mg}_{2.85} \mathrm{Fe}_{0.15} \mathrm{Si}_{2} \mathrm{O}_{5}(\mathrm{OH})_{4}+\mathrm{Mg}_{0.75} \mathrm{Fe}_{0.25}(\mathrm{OH})_{2} \\
& 57 \mathrm{Mg}_{0.75} \mathrm{Fe}_{0.25}(\mathrm{OH})_{2}+30 \mathrm{SiO}_{2}(\mathrm{aq}) \rightarrow \\
& \quad 15 \mathrm{Mg}_{2.85} \mathrm{Fe}_{0.15} \mathrm{Si}_{2} \mathrm{O}_{5}(\mathrm{OH})_{4}+23 \mathrm{H}_{2} \mathrm{O}+4 \mathrm{Fe}_{3} \mathrm{O}_{4}+4 \mathrm{H}_{2}
\end{aligned}
$$

The above reactions represent an abundant source of geological reducing power. These reactions occur at depths of roughly $2-8 \mathrm{~km}$ under the ocean floor and at temperatures between $c a$. $80^{\circ} \mathrm{C}$ and $200^{\circ} \mathrm{C}$. They provide copious amounts of molecular hydrogen for organic synthesis, and they bring dissolved carbon compounds in contact with reduced transition metals.

In serpentinization, the electrons that generate $\mathrm{H}_{2}$ and reduced carbon stem from the transition metals iron and nickel. It is highly likely that serpentinization has been taking place on Earth since there has been liquid water on the planet, and the same will be true for other wet, rocky planets (McCollom and Seewald, 2013; Schrenk et al., 2013). This process is observed in nature but has not been systematically characterized in the laboratory. Initial studies are very encouraging, with formate, methanol, acetate, and pyruvate having recently been synthesized from $\mathrm{CO}_{2}$ under hydrothermal conditions using FeS mineral catalysts (Roldan et al., 2015). More comprehensive laboratory investigation is a critical element of this key objective.

Key Objective 3. To better characterize and understand carbon reduction in modern serpentinizing hydrothermal vents. The importance of reduced carbon compound synthesis in hydrothermal systems - the prerequisite to generating the building blocks of life-is becoming an increasingly recognized property of these systems (Russell and Hall, 1997; Shock et al., 1998). A key approach to this understanding will involve laboratory experiments to simulate the organic-synthetic ability in early Earth environments (Barge et al., 2014; Herschy et al., 2014). Note that the kinds of hydrothermal systems currently in the foreground of scientific investigations are not primarily the $c a$. $350^{\circ} \mathrm{C}$ hot, "black smoker" kinds of vents, with life spans on the order of decades. The vents of interest are, rather, the more recently discovered, cooler $\left(\mathrm{ca} .70^{\circ} \mathrm{C}\right)$ and geologically more stable kinds of vents, with life spans on orders of $10^{4}$ to $10^{5}$ years. These would have been very widespread on early Earth, where they played an important role in sequestering and modifying reduced carbon species. The early terrestrial rocks document the importance of hydrothermal activity on early Earth (Hofmann and Harris, 2008; Westall et al., 2015a, 2015b).

Of the many suggestions for organic synthesis on early Earth, the only one that we can see in action, in measurable amounts, is serpentinization-dependent $\mathrm{CO}_{2}$ reduction, generating methane and short hydrocarbons in those submarine and terrestrial systems studied so far (McCollom and Seewald, 2013; Schrenk et al., 2013). On early Earth, there was more hydrothermal activity than today (Hofmann and Harris, 2008; Westall et al., 2015a); consequently, there was also much more $\mathrm{H}_{2}$ and $\mathrm{CO}_{2}$ (and methane). Hence, the capacity for geo-organic synthesis was also much greater than today. There is a need to investigate through experimentation, observation, and modeling the magnitude of this process in an early Earth context.

Key Objective 4. To better characterize and understand hydrothermal modification of carbon delivered to Earth from space. Based on the measured flux of extraterrestrial organic carbon in micrometeorites that reach the surface of present-day Earth, Maurette (2006) calculated that a huge amount of $\sim 5 \times 10^{24} \mathrm{~g}$ reached Earth's surface in the 300 million years following the Moon-forming impact. Interaction of that carbon with serpentinizing systems was unavoidable. This is a promising and, to date, unexplored avenue of pursuit (see also Research Topic 2).

The far-reaching consequences of chemical interactions between carbon from space and rock-water-carbon interactions on early Earth for the generation of life's building blocks have not yet been explored. This, and the broader characterization of organic synthesis in laboratory-scale 
simulations of hydrothermal systems, is a very high priority for experimental investigation.

Key Objective 5. To better understand the role of molecular self-organization, higher-order organization, and cellular organization in the origin of life. A property of matter crucial to our understanding of life's origin is its ability to undergo self-organization into higher aggregation states under suitable conditions. The organization of onecarbon and one-nitrogen species, for example amino acids and bases, in the basic building blocks of cells is increasingly well understood in that a variety of conditions deliver convergent results. Whether starting from methane, formamide, or atoms in space, the carbon atoms are combined into amino acids and bases (Saladino et al., 2012), as predicated by chemical thermodynamics.

Higher orders of spontaneous self-organization can be observed at the experimental level, for example, phase separation and stacking forces that lead to spontaneous, noncovalent fiber formation of nucleobaselike organics (Cafferty et al., 2013). At the theoretical level, a very rich body of work on self-organization exists (Eigen, 1971; Kauffman, 1993) that is only beginning to be tested in laboratory experiments (Vaidya et al., 2012). Prior to the advent of molecules with genetic properties (selectable inheritance), chemical organization is best described by spontaneous reactions under kinetic and thermodynamic control. Understanding energy flux through cells and the kinds of energy that run life processes (Martin et al., 2014) is salient to issues concerning the innate tendency of living matter to attain more stable states. To understand the transition from collections of complex organic molecules to more structured states with novel and possibly emergent properties, a better knowledge of spontaneous selforganization is needed.

\subsection{Approach to achieve the key objectives}

There is currently rapid convergence between geochemistry and microbiology in the context of hydrothermal origins. This is a new and unexpected development in understanding life's origins, the nature and distribution of primitive life, as well as the possibilities and requirements for its origin elsewhere. The means chosen to meet these scientific objectives should capitalize upon this convergence.

Next ten years. Forging major progress in understanding rock-water-carbon interactions in an early Earth-early life context is a goal that can be achieved within the next decade. Accordingly, 10 years from now we can expect substantial closure of the gaps that impair our understanding of the energy-releasing processes that originate in Earth's core and are at the center of terrestrial life's origin.

Study and model hydrothermal systems. Geothermal activity requires convection, hence, heat flux. The source of heat from the core can stem from radioactivity or gravity. Better understanding of hydrothermal activity requires more knowledge of processes on wet rocky planets that generate internal heat and, as a consequence, reduced carbon compounds. Hydrothermal activity had an enormous influence on rocks and fluids on early Earth, and in the process, it had an enormous influence on geo-(organo)-chemical reactions and the habitats for early life (Westall et al., 2015a, 2015b). It has only recently been discovered that serpentinizing systems generate reduced carbon compounds (Proskurowski et al., 2008). The volume of the modern oceans circulates about once every 100,000 years (on early Earth, much faster) through hydrothermal vents (Fisher, 2005). Hydrothermal systems have therefore been altering the state of carbon-all of it and all the time-since there has been water on Earth. That will continue until there is no water left; and for other wet rocky planets, there is no reason to assume that the situation is, or ever has been, different. Therefore, it is imperative to study and model the flux of ocean water through the crust via hydrothermal cycling and its effects on the cycling and fate of carbon speciation through geological time, recognizing the gradual but ineluctable decrease in the flux of energy (heat) coming from the core with time.

By studying known underwater sites such as Lost City (Proskurowski et al., 2008) or terrestrial venting sites such as Chimaera in Turkey (Etiope et al., 2011), dramatic progress in understanding can be expected within the next 10 years, especially through in situ investigations of terrestrial systems.

Laboratory studies using vent-simulation reactors (flow reactors). It is essential to investigate in more detail the nature of electron sources and catalysts, that is (in this context), metals in hydrothermal systems throughout the geological history of Earth, from its earliest stages to the present. This is a new field at the interface of organometallic chemistry, bioinorganic chemistry, and microbial physiology. The large unfilled " $d$ " (and " $f$ ") electron shells of the (inner) transition metals are an innate, natural property of these elements that allows them to hybridize in many different combinations and thus generate metastable bonds with $\mathrm{C}$ and $\mathrm{N}$ and, thereby, provide catalysis. Laboratory experiments to investigate the organic catalytic capabilities of transition metals in natural rock-water interaction contexts and in biomimetic contexts are needed.

Furthermore, early Earth can serve as a general model and as a proxy for young rocky planets. Therefore, it is necessary to study experimentally and in modeling simulations of the interactions of $\mathrm{N}, \mathrm{S}, \mathrm{P}$, and $\mathrm{O}$ species with transition metals, $\mathrm{CO}_{2}$, and $\mathrm{H}_{2}$ at the water-rock interface in hydrothermal systems. Flow reactors simulating hydrothermal vents represent a new and technically challenging opportunity.

To study molecular self-organization, flow reactors with long-term monitoring of increases in chemical complexity are needed. Suitable model laboratory systems have been reported (Baaske et al., 2007; Barge et al., 2014; Herschy et al., 2014), providing proof of principle for such investigations.

Within the next decade, laboratory vent-simulation reactors can be expected to deliver highly complex molecular assemblages and detailed insights into the role of different catalysts and combinations thereof (transition metals), with far-reaching impact on our understanding of the significance of hydrothermal systems in an origin-of-life context.

Laboratory-hydrothermal processing of meteoritic carbon. There was a substantial delivery of a wide variety of 
organics, including nucleobases, such as purine (Callahan et al., 2011) from space by meteorites, micrometeorites, and comets. Today, this process delivers on the order of 20,000 tonnes of carbon-rich micrometeorites per year to the surface. Early influx rates were much higher $\left(\sim 5 \times 10^{16} \mathrm{~g} / \mathrm{year}\right.$; Martins et al., 2013) (see Research Topic 2). Moreover, energy produced by the impacts could have modified the extraterrestrial chemical species (Martins et al., 2013). Once delivered, exogenous carbon interacts with $\mathrm{Fe}, \mathrm{Ni}$, and other transition metals in hydrothermal systems (which generate

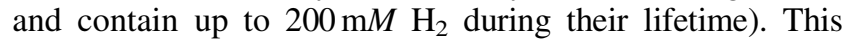
critical rock-carbon interaction process needs to be simulated in the laboratory. The major interface of carbon with reactive surfaces in aqueous environments at temperatures conducive to the accumulation of reduced organic compounds needs to be explored. There are no major technological barriers that would preclude immediate implementation of these crucial experiments. Laboratory-hydrothermal processing of meteoritic carbon should become a routine procedure within the next 10 years.

Theoretical approach. For studying molecular selforganization and its role in the origin of life, important intermediates between complex chemicals and semicellular organization have been identified in the form of autocatalytic networks (Kauffman, 1993; Hordijk et al., 2010) and stochastic replicators (Szathmáry and Demeter, 1987). It is necessary to investigate further the properties of these and related constructions that can help bridge the gap between complex organic molecules and replicating cells. Within the next 10 years, advances in the understanding of self-organization can be expected, both from the theoretical standpoint and before the experimental background of continuous laboratory-scale hydrothermal reactors outlined above.

Ten to twenty years from now. Study of extraterrestrial hydrothermal systems. On the midterm of 10-20 years, missions dedicated to the further investigation of known hydrothermal systems on Enceladus and the discovery of more, terrestrial and elsewhere within the Solar System, will provide a fuller understanding of the extent and significance of this process on rocky celestial bodies and its relationship to organic synthesis.

Laboratory studies using vent-simulation reactors (flow reactors). On the midterm of 10-20 years, we can anticipate progress in understanding the cumulative effects of synthesis within continuous flow reactors. The spontaneous synthesis of cofactorlike molecules can be anticipated. Cofactors represent a crucial threshold because, with the spontaneous synthesis of organic catalysts and continued synthesis of precursors (generated by inorganic catalysts), the observation of emergent properties within reactors becomes possible.

After twenty years. Within this timeframe, we should have a substantially clearer picture of the past presence and current distribution of serpentinizing systems within our solar system, places where microbial life-forms could potentially also have arisen, and a better general picture of the relics of planetary history that are preserved in modern microbial metabolism.
Study of extraterrestrial hydrothermal systems. On the longer term, we can envisage in situ investigations on Enceladus and related sites.

Laboratory studies using vent-simulation reactors (flow reactors). On the longer term, we can also envisage insights into the ability of laboratory systems to support the spontaneous synthesis of very complex macromolecules, and the higher-level molecular dynamics within reactors should become an object of investigation. It should become clear whether, and in which way, meteoritic carbon is essential for the synthesis of complex molecular ensembles.

Theoretical approach. Theoretical approaches involving improved understanding of organic self-organization, one of the most challenging frontiers of science, can be anticipated. Figure 5 gives an integrated view of Research Topic 3 together with its timeline.

\subsection{European strengths and needs}

Europe has a high concentration of expertise in the following areas, which are of pivotal importance for achieving progress on these objectives:

- Early Earth geological and geochemical settings;

- The nature and habitat of early life;

- Redox disequilibria;

- Serpentinizing systems in nature and in laboratory simulations;

- Anaerobic autotrophs and how they generate reduced iron from $\mathrm{H}_{2}$ (electron bifurcation);

- Autotrophic origins, hydrothermal vents, bioenergetics of anaerobic microbes;

- Theory and principles of higher-order self-organization.

One of Europe's greatest strengths in this general field has been plurality of views and the close interactions between biologists and geologists. While the view of "prebiotic broth" long stood at the center of thinking on life's origin, current textbook views for the origin of life have hydrothermal systems, rock-water-carbon interactions, and transition metal catalysis in the foreground (Madigan et al., 2014), and further exploration of metabolism and geochemical processes will continue to uncover new insights into the problem. The interactions between geologists and biologists in Europe represent a particularly important infrastructural and intellectual strength [EANA, Mars Analogues for Space Exploration (MASE), AstRoMap, for example]. The hydrothermal theory for the origin of life (Baross and Hoffman, 1985; Russell and Hall, 1997; Wächtershäuser, 2006; Baross and Martin, 2015) stems almost entirely from European scientists and has, in its current formulations, methane generation as the most ancient form of microbial physiology among archaea (Lane and Martin, 2012), forging a link to spontaneous geochemical processes. The production of methane in modern terrestrial serpentinizing systems and the recent claims for detection of methane on Mars (e.g., Webster et al., 2015) emphasize the importance of being able to discriminate between biological and geochemical methane production in rock-carbon interactions.

Furthermore, the convergence between geochemistry and microbiology is more tangibly manifested in Europe than 


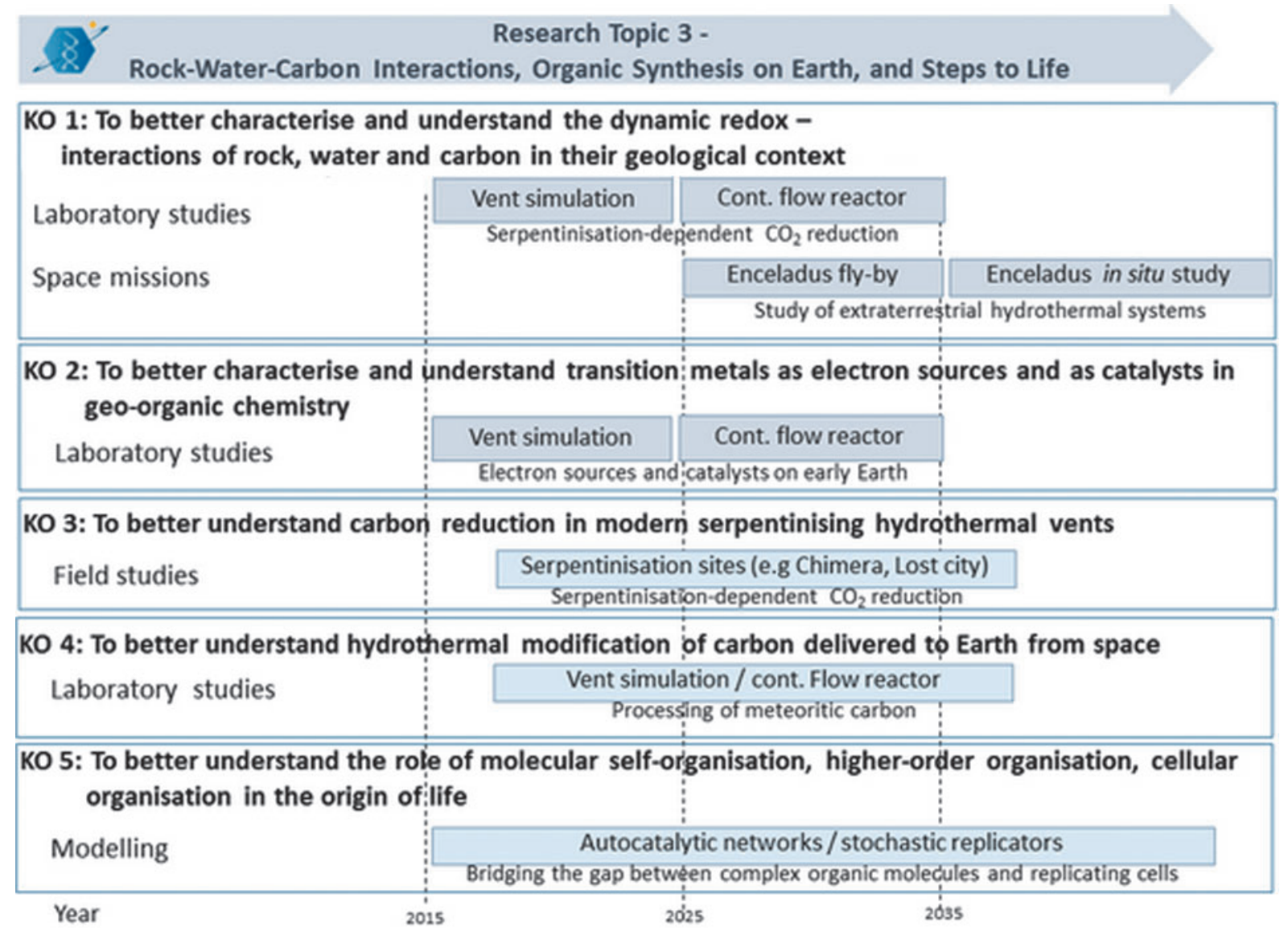

FIG. 5. AstRoMap Roadmap, approaches to reach the key objectives of Research Topic 3 "Rock-Water-Carbon Interactions, Organic Synthesis on Earth, and Steps to Life" within the next 10, 20, or follow-on years.

anywhere else in the world. Europe should capitalize upon this convergence.

However, the realization that modern hydrothermal systems catalyze organic synthesis-and that early Earth hydrothermal systems did as well-is relatively new, within the last couple of decades. The implications of this impor- tant advance have not yet been assimilated into the intellectual fabric of the field. This requires a transformative advance in understanding with broad implications for progress in planetary evolution and investigations of early life. To achieve this requires dissemination of information and demonstrating proof of principle.

\section{Research Topic 4: Life and Habitability}

\subsection{State of the art}

The history of life on Earth spans at least 3.5 billion years, as studies of microfossils in the oldest sedimentary rocks have revealed (reviewed in Westall, 2011). Since their emergence, prokaryotic microorganisms continued to shape Earth's biosphere during the first nearly 2 billion years of life's history, before the first unicellular eukaryotes (cells with nuclei, flexible membranes, and cytoskeletons) appeared (Javaux, 2007). Microbial evolution occurred and continues to take place under a vast variety of conditions that range from anoxic to oxic, hot to cold temperatures, low to high $\mathrm{pH}$, free-living to symbiotic, and so on (Kristjánsson and Hreggvidsson, 1995; Bakermans, 2015). As a result of this supremacy of the microbial world, microorganisms defied natural catastrophes and never became extinct (Cockell, 2003).
Our ability to explore the evolution of Earth's biosphere and its boundaries has increased tremendously during the last few decades. Since the discovery of the first extremophile, Thermus aquaticus, from thermal vents in Yellowstone National Park in the United States (Brock and Freeze, 1969), scientific expeditions have discovered an astonishing diversity of extremophiles (prokaryotes, eukaryotes, and viral particles) in a variety of extreme environments. Such environments include deep-sea hydrothermal vents, the Dead Sea, cold saline lakes in Antarctica, deserts, subterranean ecosystems, and habitats within rocks (Rothschild and Mancinelli, 2001; Le Romancer et al., 2007; Stan-Lotter, 2007; Bell, 2012; Chan et al., 2012). Several types of polyextremophilic organisms have been found with most remarkable features. For example, saturated fatty acids in the cell membrane enable hyperthermophilic organisms (such as Pyrolobus fumarii) to persist also within high- 
pressure environments (Blöchl et al., 1997). Organisms that can survive dehydration (anhydrobiotes), such as cyanobacteria and tardigrades, are also often adapted to both desiccation and extreme temperatures, and many of these can resist prolonged exposure to conditions encountered in space (Horneck, 1993; Horneck et al., 2010; Billi, 2012; Onofri et al., 2012). Even more impressive are the survival capabilities of the dormant states of different species; for example, bacterial spores undergo no detectable metabolism and exhibit a higher degree of resistance to inactivation by various physical challenges, including (but not limited to) wet and dry heat, UV and gamma radiation, extreme desiccation (including vacuum conditions), and oxidizing agents (Nicholson et al., 2000). These discoveries have greatly improved our understanding of the evolutionary potential of life and the habitability of different environments on Earth. They have also raised puzzling questions about the mechanisms of cellular survival, aging, and the multitude of factors that drive the adaptation, evolution, and diversity of life and the environments that sustain it, as well as the parameters needed to make a certain environment habitable (Cockell, 2007; Wassmann et al., 2010, 2012; Bauermeister et al., 2014). Although research on natural life systems should be prioritized since they are far from well explored, synthetic biology is also anticipated to yield further novel insights, for example, via the engineering of complex artificial systems for investigating natural biological phenomena (Andrianantoandro et al., 2006).

Parallel to research into extreme ecosystems on our planet, space exploration has paved the way for studying presumably habitable extraterrestrial environments (Lammer et al., 2009). However, efforts to characterize the habitability of these environments or even to search for indigenous life have encountered several challenges, including abiotic artifacts, technological and methodological limitations, and a lack of sufficient knowledge of the environmental conditions when designing experiments prior to a mission. This is exemplified by the Viking missions to Mars, which searched for evidence of the metabolic activity of potential soil microbial communities (Klein, 1978). Although most experiments appeared to give positive results, they could be, and were finally, explained by nonbiological phenomena, namely, the chemical reactivity of peroxides present in the regolith that were capable of decomposing organic molecules (Yen et al., 2000) (also see Research Topic 5). The follow-on missions to Mars (Mars Exploration Rovers and Mars Science Laboratory) more cautiously approached the question of life (extant or extinct) on Mars by searching for indirect indications of biological activity (for example, based on the principle of "Follow the Water'-Grotzinger et al., 2014). Even negative results from such missions must be carefully interpreted since these could simply reflect inappropriate sampling strategies or inadequate interpretation due to limited knowledge. Since 2001, the Mars Exploration Program Analysis Group (MEPAG) regularly advises NASA on the scientific priorities for investigations to be carried out by the robotic Mars flight program (MEPAG, 2015). The ExoMars mission, ESA's next step in Mars exploration, is designed to investigate the habitability of past and present Mars and search for signatures of extinct life (Vago et al., 2006, 2015; Vago and Kminek, 2007).

Among the icy moons of the outer planets, Jupiter's moon Europa is of special interest to astrobiologists because it may have a liquid ocean beneath its icy crust, which also may be in contact with a silicate layer (Sotin and Prieur, 2007). The recent hints of water plumes at the south pole of Europa suggest convection of material from below the ice onto its surface (Roth et al., 2013). In addition to Europa, Saturn's moons Titan and Enceladus are candidate targets for astrobiologists (see Research Topic 5 for further details). Continuing observations of the Cassini-Huygens mission are beginning to reveal the mystery behind the seas and lakes on Titan, which are filled with liquid hydrocarbons (Cornet et al., 2015). This mission has also generated important new insights concerning the subsurface ocean, plumes, and geophysical activities of Enceladus (Hansen et al., 2006) (see Research Topic 3). With the increasing number of exoplanets being observed and confirmed (see Research Topic 1), the quest for habitability has now extended beyond our solar system to the galactic habitable zone (e.g., Gonzalez, 2001; Lineweaver et al., 2004).

Beyond data from orbiters or landers, much information has been gained from studies on terrestrial analogues of putative extraterrestrial habitats (e.g., Horneck, 2000; CAREX, 2011a; MASE, 2014) and conditions that simulate those environments (e.g., Pogoda de la Vega et al., 2007; de Vera et al., 2010; Noell et al., 2015).

Thus, the last decades of research have opened up many novel, interesting possibilities. To adequately approach these opportunities, it is pivotal that current constraints are overcome and an interdisciplinary investment is undertaken to support further research both on Earth and in space by different scientists from different scientific and technological fields. To this end, we suggest three key scientific objectives, as outlined below.

\subsection{Key objectives}

Key Objective 1. To expand our knowledge of the diversity, adaptability, and boundary conditions of life on Earth. Despite the wealth of scientific knowledge that has accumulated over the past decades, our understanding of the diversity of life and the parameters that define habitability remains incomplete. Addressing this knowledge gap is pivotal to understanding the evolution of life on Earth, identifying essential parameters that characterize a habitable environment, planning sampling strategies and analytical methods for future space missions, and interpreting the results from these efforts. There is a requirement, therefore, for further research on Earth-based life that encompasses several scientific fields, as well as for substantial improvements on current sampling, analytical technologies, and the establishment of high-quality databases. Creating an interdisciplinary link between these fields will optimize the joint research ventures aimed at understanding the origin and fate of life on Earth, identifying habitable extraterrestrial environments, and establishing appropriate concepts for planetary protection issues. To achieve this, our current definitions of life and habitability must be revised and expanded. 
The most basic requirements for life on Earth, as we know it today, include liquid water, a source of energy and carbon, and macroelements and trace elements. Physical and chemical extremes can, however, limit the capacity of an environment to sustain biological processes. While the concept of habitability varies across disciplines (see Section 1.3.2. Approaching habitability), this topic focuses on the boundaries and evolutionary potential of life and the habitability of different environments. This also raises the question as to how these coevolve over time and how this knowledge can be used to identify the general principles of habitability on Earth and elsewhere. Our knowledge of the boundaries for life on Earth defines the physicochemical parameter space within which we can expect to find actively growing and reproducing organisms. The standard parameters that define these boundaries include, for example, extremes of temperature, $\mathrm{pH}$, salinity, pressure, redox states, radiation, gravitation, the availability of electron donors and acceptors, and thermodynamic laws (Hoehler, 2007; Stan-Lotter, 2007; Stevenson et al., 2015). Some extreme environments are characterized by only one or two of these parameters. However, several environments are characterized by multiple extremes (Harrison et al., 2013). These environments can therefore be regarded as more challenging (as well as of particular interest) because they reflect the conditions likely to be found within extraterrestrial environments, as well as conditions on early Earth when the first steps towards the origin and development of life took place (Westall et al., 2013).

Since our own planet is far from well explored, with respect to both different types of ecosystems and the total number of estimated biological species and viral particles, it is imperative that we continue to explore different types of environments. This will improve our knowledge about the diversity of life on our own planet at present and, wherever possible, in the past. To expand upon earlier research approaches, it is crucial that we develop improved sampling techniques and analytical tools, based on a critical reevaluation of former strategies and hypotheses. It is furthermore important that more systematic, holistic multidisciplinary analyses are performed, which are aimed at retrieving molecular data and improving our methods of cultivating organisms and viral particles from all domains for in-depth explorations of their biology. These data should be followed up by advanced models and simulation-based approaches (including synthetic biology) to evaluate our understanding of the physical and chemical limits for life on Earth on a global scale. To achieve this, strong collaborations between different scientific fields will be needed.

Sub-objective 1. To explore the diversity of life on Earth. Since only a fraction of the total biodiversity (including viral particles) of the different ecosystems on Earth has been explored, investigations of these should be continued with more advanced methodologies to address research questions that have been impossible to address to date. While it is logical to focus on extreme ecosystems since only a fraction of these have been explored, it is nevertheless important to systematically compare these systems with mesophilic ecosystems. This will enable us to improve our knowledge of the origin, diversity, and limits of life and the habitability of different environments on Earth and elsewhere. Of special interest are multiple-stress habitats since these are likely to better represent the conditions for life on early Earth, as well as the extreme conditions encountered in other parts of our galaxy. Although research into multiple-stress habitats is fundamental to exploring the origin and the boundaries of life, surprisingly little is currently known about these environments. One of the key research avenues, therefore, is to continue research on such ecosystems and explore how adaptation to multiple-stress conditions influences the physical and chemical limits for life on a global scale (e.g., Harrison et al., 2013, 2015).

Sub-objective 2. To explore biological interactions and systems ecology. Biological interactions (including symbioses) have played a fundamental role in the evolutionary progress of life on Earth and expansion of the habitability of our planet. Collectively, these enabled development towards a higher degree of biological complexity (Lyons and Kolter, 2015). Examples include the endosymbiotic theory for the origin of mitochondria and chloroplasts, which initiated the development of eukaryotic cells (Margulis, 1970; Zimorski et al., 2014; Ku et al., 2015) and formation of multispecies communities and novel organisms, such as the first microbial biofilms (Westall et al., 2006a, 2006b, 2011), lichens, and mycorrhizae. However, detrimental interactions and events such as infections caused by viral particles, pathogens or parasites, or natural catastrophes have also been important to the development of novel protective strategies and the survival of life in general. Many of these interactions are mediated by elaborate processes such as horizontal gene transfer, and advanced cellular sensing and communication systems (Thomas and Nielsen, 2005; Relman, 2008; Albuquerque and Casadevall, 2012; Li and Nair, 2012). With the emergence of more complex ecosystems and the accompanying population dynamical processes, new challenges also arise, such as the accumulation of detrimental metabolic waste-products (including oxygen) and deprivation of energy and food sources that may alter or even reduce the habitability of an environment. Many of these types of interactions can also either facilitate or endanger the persistence of life within extreme environments (e.g., Rothschild and Mancinelli, 2001). Exploring all these types of interactions throughout different developmental stages (early versus more advanced ecosystems) in extreme as well as non-extreme ecosystems is therefore essential to our understanding of the evolution and survival of life from a systems ecological perspective.

Key Objective 2. To expand our understanding of the general principles of life and habitability. A variety of biological processes is fundamental to cellular development, survival, and evolution, often in interaction with the surrounding environment. These processes cover all aspects of the life cycle-reproduction, birth, growth versus regulation, aging versus rejuvenation, morphogenesis versus the degradation of cellular matter (e.g., apoptosis), death and transformation of cellular matter in the environment for the benefit of other species (e.g., Elmore, 2007; Zheng et al., 2013). They also encompass fascinating strategies for, for example, movement, dispersal, defense, sensing, communication, survival, individuality, memory, learning, heredity, and evolution in general (e.g., Neuman, 2006; Ackermann, 2013; Shimkets, 2013; Barrangou and Marraffini, 2014; Ramamurthy et al., 2014). Although these processes are 
crucial to our understanding of life in general and its evolutionary potential in interaction with the surrounding environment, they are, despite all progress in "omics" approaches and mapping correlations between specific traits and genes, far from well understood (see, e.g., Maher, 2008). It is therefore imperative to explore these in further detail in extremophiles as well as mesophiles. To enable a more holistic interpretation of these observations, it is also important to consider the developments of other scientific fields, such as physics (Bohm, 1980). With this, a more fundamental reevaluation of the scientific-philosophical discussions on physics, biology, and science in general, as initiated by E. Schrödinger (1944) and J. Lederberg (1960), can be taken up again to reduce current gaps between different scientific fields and thus enable a new approach towards a unified understanding of life and the Universe.

Key Objective 3. To assess the habitability of extraterrestrial environments. Since Mars, the icy moons, and certain exoplanets appear to be possible candidates for containing habitable environments beyond those observed on Earth, we suggest the following research tasks:

- Mars: Continued research efforts on Mars are vital to improving our knowledge about the potential for habitable conditions beyond Earth. Addressing where and when habitable conditions occur, may have occurred, or will occur on Mars will entail searching for locations where liquid water was present in association with hydrothermal activity on a timescale long enough for life to have emerged (approximately $10^{5}$ to $10^{6}$ years) (see Research Topic 5).

- Icy moons of Jupiter and Saturn: A further goal for improving our possibilities to discover habitable environments beyond Earth will be to determine whether liquid water was, or is, present on these icy moons, since any icy body that possesses a rocky core must have had a period of time when liquid water was in direct contact with it. Another important goal is to understand the geo-physical-chemical composition and history of the vents or plumes on the moons Europa and Enceladus (see Research Topics 3 and 5).

- Exoplanets: Detailed atmospheric studies of exoplanets are needed to determine whether they are likely to lie within the "habitable zone" around their star and if they could support liquid water (see Research Topics 1 and 5 for more detail). Other essential research tasks include improved attempts to determine the age of exoplanets and the role of other parameters (e.g., planetary mass, density, class of central star) upon the existence of extraterrestrial habitable environments.

Furthermore, to expand our knowledge of the boundaries of life on Earth and the habitability of extraterrestrial environments requires different types of ground-based simulation facilities as well as space experiments (e.g., on the ISS) (Horneck et al., 2010; EXPOSE-E, 2012; EXPOSE-R, 2015). These will provide us with unique opportunities to explicitly test how our knowledge of life can be extrapolated to different conditions in space. They will also help us establish appropriate criteria to define habitability and improve our ability to identify extraterrestrial environments that could support either past or present life. The next two decades will provide us with unique opportunities with respect to novel satellite-based technologies (e.g., nanosatellites) (Nicholson et al., 2011) and novel research possibilities relating to the exploration of Mars, icy moons, and exoplanets.

This key objective includes the following four subobjectives, as listed below:
Sub-objective 1. To determine and investigate terrestrial analogues for putative extraterrestrial habitats
Sub-objective 2. To determine the limits for growth and survival of life under simulated planetary conditions, in the laboratory and in space
Sub-objective 3. To explore the potential of synthetic bi- ology for future exploratory missions
Sub-objective 4. To provide basic data for planetary protection efforts

\subsection{Approach to achieve the key objectives}

In recent years, research on extremophiles has reached astonishing and unexpected results and expanded our concept of the limits of life. In parallel, space exploration has shown us that beyond Earth there are, and were, conditions that are very close to our current concepts of terrestrial limits of life. We can therefore assume that these two fields of investigation will closely overlap within the near future.

Next ten years. Activities deemed necessary within the next decade are listed below:

\section{(Micro)biological studies in the field and laboratory.}

- Improve sampling methodology, analytical methodologies, enrichment, cultivation, and maintenance of different types of organisms (micro/macro) that represent all three domains (Archaea, Bacteria, Eukarya) as well as of different types of viral particles.

- Expand screening efforts of known and novel extreme (and where appropriate also mesophilic) ecosystems of different categories (characterized by one, two, or several extreme parameters), including biodiversity and systems ecological studies.

- Conduct experimental research into the physiology and ecology of extremophiles by means of "omics" approaches, focusing on complex geobiological systems under a broad range of conditions.

- As a consequence of bioprospecting and research on novel species, develop novel inventions for biotechnological applications (see Section 7.1.1).

(Micro)biological experiments in space and in the laboratory under simulated extraterrestrial conditions (of space and planets).

- Perform experiments on the ISS and on satellites (such as nanosatellites), for example, with regard to survival of different species during spaceflight.

- Improve our knowledge of extraterrestrial environments via ground-based simulations and modeling of habitable conditions on other planetary bodies (e.g., icy moons). 
- Extend our understanding of key determinants of planetary habitability via ground-based simulations, modeling, and robotic exploration of habitable conditions on Mars.

- Expand current concepts for planetary protection.

Data management and archiving.

- Construct interdisciplinary databases of high quality for microbiological and molecular biological studies.

Synthetic biology and modeling.

- Promote synthetic biology and advanced modeling with a view to improving our understanding of evolutionary adaptations to extreme conditions and how these enable life to persist within biologically hostile environments.

\section{Interdisciplinary communication and collaboration.}

- Improve communication between different types of relevant research laboratories via scientific societies (e.g., EANA), joint expeditions, collaborations, and international projects.

- Promote interdisciplinary research on extreme environments.
- Increase collaboration with scientists who are not directly working in the field of astrobiology to promote novel approaches to habitability research, as well as technological inventions and further practical integration between fields (including life sciences, geosciences, physics, engineering, and the humanities, e.g., fields addressing ethical-philosophical or psychological issues).

- Promote a creative exchange between different scientific-philosophical and humanistic disciplines in order to bridge the gaps between those disciplines that aim to identify different means of describing the fundamental nature of the Universe.

Ten to twenty years from now. Within this time span the following activities should be performed:

- Large-scale expeditions to extreme locations.

- Developing a more comprehensive overview of the diversity of life, viral particles, and the variety of nonextreme as well as extreme environments on Earth.

- First steps towards complex and systems-based geoecological models (experimental and theoretical) of multi-extreme ecosystems.

- First steps towards complex models concerning the origin and evolution of cell-based life and viral parti-

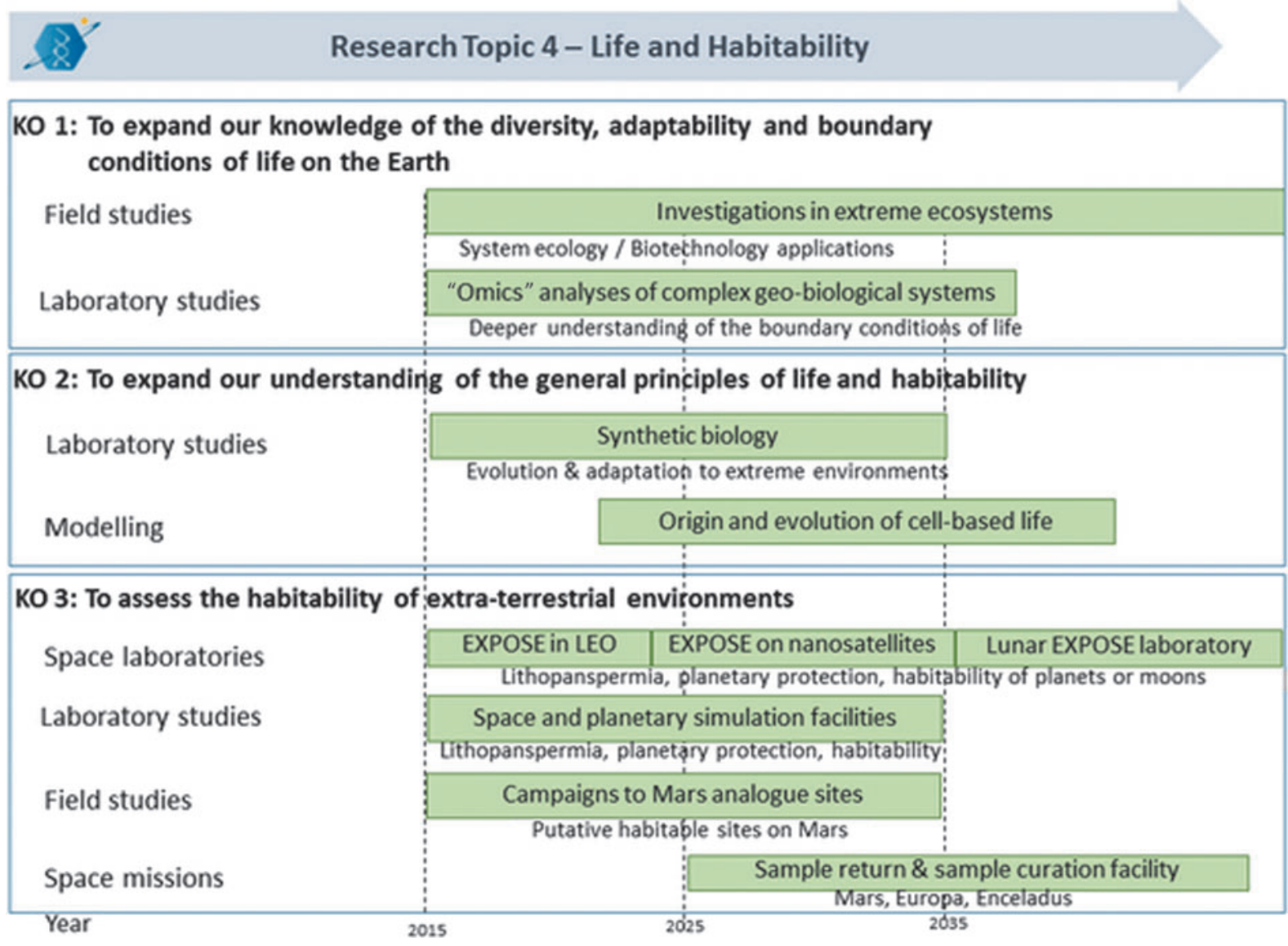

FIG. 6. AstRoMap Roadmap, approaches to reach the key objectives of Research Topic 4 "Life and Habitability" within the next 10, 20, or follow-on years. 
cles, and what factors determine habitability of a given environment.

- Analysis of original samples from different locations on Mars.

After twenty years. After 20 years we should have reached the following state of knowledge:

- A deeper understanding of the physical and chemical boundaries of life (including viral particles) on Earth and beyond.

- Extended knowledge of habitability obtained through the exploration of other planetary bodies (e.g., icy moons and exoplanets).

- Expanded concepts for planetary protection.

- First steps towards adequate considerations for biotechnological applications in space (e.g., space mining, ecopoesis, in situ resource utilization in support of human settlements, e.g., food production).

- Return and analysis of original samples from Mars and different icy moons.
Figure 6 gives an integrated view of Research Topic 4 together with its timeline.

\subsection{European strengths and needs}

Europe has a large and diverse scientific community in astrobiology due to different cultures and countries. Experts in different fields are actively studying diverse aspects of extreme environments, spanning disciplines such as astronomy, biology, chemistry, geology, physics, medicine, space technology, and biotechnology. There is strong expertise within several European laboratories in organizing large-scale expeditions, especially to places analogous to putative habitable extraterrestrial environments, as well as in sampling, analysis, and evaluation of probes from those extreme environments. For optimal accomplishment of the suggested research key objectives, it is necessary to initiate more interdisciplinary cooperation between all these different research directions and facilities on a larger scale. Part of this work has already been accomplished via EANA (http://www.eana-net.eu).

\section{Research Topic 5: Biosignatures as Facilitating Life Detection}

Preamble: We note that, for the geological and biological communities, the term "biomarker" refers to an organic molecule whose origin can be directly related to an organic component of life, even when degraded. "Biosignature," however, refers to a broad range of signatures of life, morphological, geochemical, and organic, that may not necessarily be unambiguous. In the atmospheric modeling community, on the other hand, the terms "biomarker" and "biosignature" are usually used interchangeably to indicate a signal that can only be produced by life whereas "bioindicator" refers to a signal that is indicative of life but is not unambiguous.

\subsection{State of the art}

The signatures of life (biosignatures) are extremely diverse and may be related either to living organisms or to the (fossilized) remains of extinct organisms. They comprise

- Disequilibria, for instance in redox conditions or in the thermodynamic state of the system;

- A relatively limited range in the composition of organic molecules used in a cell (note that the same molecules may occur in extraterrestrial organic matter, although their compositional range is far wider than that used by life);

- A pronounced chiral signature of the organic molecules used by life;

- Specialized molecules such as DNA, RNA, lipidic acids, proteins, and so on;

- Fractionated isotopes of elements such as C, S, Fe among others (although note that abiotically produced isotopic signatures may overlap with those produced by life, but the range of abiotic isotope signatures is far broader than that of biological signatures);

- Gases related to biological metabolisms, such as oxygen produced by oxygenic photosynthesis or methane produced by methanogenic microorganisms, both of which may also be produced by abiotic processes;

- A characteristic spectral signature, for example, the "red edge" for vegetation;

- Leaching of elements from minerals and their concentration, especially metals, by exopolymeric substances;

- Biominerals formed as a result of microbial metabolisms, for example, carbonate, phosphate, iron oxides, and sulfides (again, these minerals may also be formed by abiotic processes);

- Physical microbial structures including biolamination or stromatolites and mud mounds. These features are formed by precipitation of minerals or trapping of detrital particles by exopolymeric substances. Microbially induced sedimentary structures come under this category.

The above constitute only a few from a huge list of potential biosignatures (see recent review in Westall and Cavalazzi, 2011). A challenging issue related to biosignatures is that they can occur over a wide range of spatial and time scales, with further modifications due to contextual conditions. For example, the fossilized remains of individual microorganisms may be of the order of micrometers (e.g., Knoll, 1985), but biological constructions implicating microbial conditioning of the environment may extend to scales of the order of meters to hundreds of meters or more (e.g., Noffke, 2009). Examples of the latter are exposures of large-scale (meters to tens of meters) stromatolitic constructions (e.g., Kalkowsky, 1908), or mud mounds (Riding, 2011). The nature of the fossilized organisms has changed over geological timescales, first anaerobic, single-celled microorganisms, then multicellular aerobic organisms of varying sizes and complexity, and finally technologically developed beings. Each of these types of life-form has left its signature on Earth on scales of the order of microns to those encompassing the whole planet. Early methanogens, although single-celled and anaerobic, may have contributed to producing enough $\mathrm{CH}_{4}$ in the atmosphere to create a greenhouse effect at the time 
when the early Sun was less luminous (Ueno et al., 2006; Gaillard and Scaillet, 2014). The appearance of oxygenic photosynthesizers led to the oxidation of Earth's surface and, consequently, the simultaneous appearance of disequilibria redox gases in the atmosphere (e.g., oxygen and methane). Human activity since the end of the 19th century has been contributing to human-made perturbations to the atmosphere and the climate in general.

Biosignatures cover a wide range of spatial and time scales. On the microbial scale, there has been much study on biosignatures related to investigation of the traces of life on Earth through geological ages. In this context, study of the oldest traces of life underline the inherent difficulties in distinguishing biogenicity and syngenicity of potential biosignatures. Nevertheless, in the geological and biological communities, understanding of the nature of biosignatures has been growing steadily over the last decades as a result of better knowledge of the preservation of signatures of life through experimentation and observation (Orange et al., 2009; Westall and Cavalazzi, 2011; Benzerara et al., 2014; Oehler and Cady, 2014), for example, of the effects of microbial activity on its immediate mineralogical environment (Bennet and Omelon, 2011; Röling et al., 2015) and, not least, because of the availability of instrumentation with increasing powers of resolution (Miot et al., 2014). The applications of this increased understanding are widely applied to the search for the oldest traces of life on Earth and for potential extraterrestrial biosignatures on Mars (Noffke, 2015).

Second, on the (exo)planetary scale, the last 10 years have seen an enormous expansion in modeling studies that focus on atmospheric biosignatures. These include investigations of the appearance of oxygenic photosynthesis and its impact on the globe via the rise in atmospheric oxygen, as well as biosignatures of relevance to the search for life on other planets, for instance, Mars, as well as exoplanets. In the exoplanetary atmospheric modeling community, Kasting et al. (1993) produced a cornerstone study investigating Earth-like atmospheric responses in climate over the habitable zone. Their work has been built upon by numerous studies including those of Segura et al. (2003), Rauer et al. (2011), and Grenfell et al. (2014), all of whom investigated the effect of varying the amount of oxygen or planetary mass on biosignatures. Kaltenegger et al. (2007) calculated theoretical atmospheric spectra over geological timescales. Recently, numerous 3-D modeling studies have investigated the effect of planetary climate, dynamics, and their influence on the width of the habitable zone (e.g., Leconte et al., 2013; Shields et al., 2013).

Two central goals in biosignature research are as follows:

(i) to distinguish true life signatures from abiotic "false positives" that merely mimic life;

(ii) to avoid situations where real biosignatures are overlooked ("false negatives") because their signals may be weak or masked, for instance, by a thick layer of cloud. These concepts were discussed by Tarter et al. (2007) and references therein.

One famous example may be cited here to illustrate the thorny question of distinguishing truly biogenic signatures from abiotic mimics in the search for life on Mars. The Viking mission consisted of two landers containing instruments to detect extant life. They landed on opposite sides of the planet, Viking 1 in Chryse Planitia and Viking 2 in Utopia Planitia in
1976. Both landers carried a suite of experiments to detect traces of extant life, which consisted of (1) a gas chromatographmass spectrometer (GC-MS), (2) a gas exchange experiment, (3) a labeled release experiment, and (4) a pyrolytic release experiment. While carbon molecules were not detected by the instrumentation, the labeled release experiment did detect the release of labeled $\mathrm{CO}_{2}$, a result that was initially welcomed by the experimenters as evidence for extant, metabolizing life on Mars (Levin and Straat, 1976). However, taking the results of all the experiments together, the final general consensus was that there was no evidence for extant life. The reasoning was based on the fact that, even if no life were present, organic molecules introduced continuously by the same constant "rain" of carbonaceous meteorites and micrometeorites as occurs on Earth should have been present on the planet. No carbon of either abiotic or biological origin was detected; therefore the results of the labeled release experiment must have been due to a non-organic and nonbiological phenomenon (e.g., Horowitz et al., 1976). Nevertheless, two recent findings over the last decade show that the analysis of the martian organics and potential life was more complicated than initially envisaged in 1976. Firstly, the instrumentation on the Viking landers did not have sufficient resolution to detect microbes in, for example, the desert soil of Atacama (admittedly at very low concentrations, Navarro-González et al., 2006). Secondly, the detection of perchlorates (Hecht et al., 2009) in the martian soil at the Phoenix landing site indicated that any organic molecules present in martian rocks and soil would be oxidized upon heating in the GC-MS instruments. Nevertheless, in the ongoing Mars Science Laboratory mission at Gale Crater, careful backtracking has allowed the Sample Analysis at Mars (SAM, an instrument that includes a GC-MS) instrument team of the NASA Curiosity rover to confirm the presence of chlorobenzene in martian rocks (Freissinet et al., 2015).

Although many investigations have been undertaken to date, there are certain key topics that need to be addressed in more detail and in a more concerted, coordinated manner, as outlined below.

\subsection{Key objectives}

Key Objective 1. To distinguish life from nonlife. This concept covers phenomena ranging in scale from prebiotic molecular features (e.g., micelles) to planets ("dead" planets versus "live" planets), focusing on life as we know it (as opposed to "weird life"). It also covers both past and present situations, for example, from ancient Earth or ancient Mars (extinct situation) to the subsurface of Mars today (possibly extant situation).

Sub-objective 1. Biological context. List and prioritize the characteristics that distinguish life from nonlife, for example, morphology, organo-geochemical chemical composition, energy budget and disequilibria in general, metabolic signatures on various scales, from microbial to global (e.g., atmospheric composition). This topic overlaps with the fundamental scientific question: What is life (Pross, 2012)?

Sub-objective 2. Environmental context. Improve knowledge of geological and environmental conditions conducive to the origin of life, for example, the importance of 
hydrothermal activity, as discussed in Research Topic 3, as well as hosting life (Westall et al., 2013). Explore the role of the coexistence of carbon, liquid water, and chemical disequilibria (in the presence of transition metals). For example, cell-like structures (e.g., micelles) located in environments with no disequilibria (no redox couples) are probably abiotic.

Sub-objective 3. In situ science. Many of the features to be studied in situ are microscopic in scale; therefore a key goal is to construct new space instrumentation that combines existing techniques, for example, between microscopy and Raman and laser-induced breakdown spectroscopy (LIBS) at microbe-relevant scales (micrometric at a minimum). The unique advantages of this approach would be the ability to analyze the same "spot of interest" with different and complementary techniques. Remote sensing from ground and from space is made challenging because the biological and geological context on the microscale is also an important aspect. Such a context is currently far from the reaches of modern technology in terms of exoplanetary research but, nevertheless, should be considered as future work (e.g., remote Raman, IR, and other spectroscopic techniques: analyses at kilometric distances to study the local context).

The following observational methods are deemed necessary:

Remote methods

- Biosignature determination via atmospheric spectroscopy;

- Estimation of chemical disequilibrium, that is, the presence of both reducing and oxidizing species;

- Determination of the presence of potential biominerals at a planet's surface, for example, carbonate;

- Estimation of isotope ratios;

- Detection of the "red edge" signal typical of photosynthesizing vegetation;

- Estimation of the presence of chiral species in an atmosphere by spectropolarimetry (Sparks et al., 2005);

- Estimation of the mid-IR galactic excesses as an indication of galactic "supercivilizations" (Griffith et al., 2015) that may be capable of reprocessing starlight into mid-IR light. Based on a study using the Wide-Field Infrared Survey Explorer (WISE) instrument, searching for mid-IR excess, certain target galaxies have been proposed for further study, for example, Arp 220.

- Biosignatures for more advanced, industrial, or technologically advanced life-forms (Lin et al., 2014) involving the spectroscopic detection of industrial pollution such as CFCs have also been proposed.

In situ methods. Here, the spatial scales typically vary from microns to hundreds of meters. Key techniques include gas chromatography, mass spectrometry, Raman, LIBS, and IR for structural investigation, chemical isotopic studies, as well as optical observation from the macroscopic to the microscopic scale. However, in terms of size, the small scale (size and intensity of signal/signature) may be a limiting factor for in situ research. This fact underlines the necessity of sample return missions, for instance, from Mars, to determine whether potential features of interest (most likely samples with a carbon signature suggestive of life) are abiotic or biotic.

The search for extant or extinct life influences the kind of techniques used. The controversy over the Viking mission results noted above (Levin and Straat, 1976, 1979) stimulated the desire to continue the search for life on Mars and determine its habitability (Westall et al., 2015b). Techniques for the identification of extant life are based on metabolic activity. In an exoplanetary context, we anticipate that the discovery of extant (as opposed to extinct) life will be highly favored since current results suggest that (Earth-like) atmospheric biosignatures are removed relatively quickly (in geological timescales) on planets where life no longer exists; oxygen will be removed from the atmosphere by reaction with the surface of the planet on a timescale of several thousands of years, while $\mathrm{N}_{2} \mathrm{O}$ and $\mathrm{CH}_{4}$ are oxidized in the atmosphere within hundreds of years (Houghton et al., 2001).

The potential for life on Titan has been discussed (e.g., McKay and Smith, 2005), although the lack of direct contact between liquid water and hot rock, as an energy and nutrient source, would surely inhibit life as we know it. In the case of Titan, some key challenges include better constraining complex hydrocarbon chemical formation rates and interaction with cosmic rays at cold temperatures. Other planetary bodies of astrobiological interest include Saturn's moon Enceladus, about which the nature of volcanic ice plumes and their indication of the presence of a salty ocean have been recently discussed (e.g., Postberg et al., 2011). Jupiter's moon Europa has long excited astrobiological interest; a recent study suggests that the dark regions on its icy surface may be evidence for an underlying salty sea (Hand and Carlson, 2015).

As a potential biomarker, atmospheric methane on Mars has triggered important discussion. Earth-based and orbital detections (Formisano et al., 2004; Mumma et al., 2009) of this gas on Mars have been reported and debated (Zahnle et al., 2011) over the past decade. But recently methane was detected with the SAM instrument on the Curiosity rover (Webster et al., 2015). The ExoMars TGO mission in 2016 contains several instruments devoted to the search for methane and other gases in the atmosphere of Mars at low concentrations.

Key Objective 2. To follow the energy: Identify energy sources, redox couples, and photoreactions. Energy is one of the three fundamental elements for the existence of life; therefore, we propose to follow redox pairs (e.g., hydrogen, iron) in a geological context (Hoehler, 2007; Hoehler and Westall, 2010) and other energy sources (e.g., emitted from a star or the planetary interior) in an exoplanetary context as a biosignature/bioindicator to determine potential habitability, noting that the existence of life can lead to disequilibria, for example, in atmospheric chemical composition or/and the planetary entropy budget.

Sub-objective 1. Geological and mineralogical context. Achieving this sub-objective would require the following key questions be addressed:

- Are redox coupling and redox disequilibria available as a source of available energy for life?

- How critical is the presence of water (hydration absorbed water) as a disequilibria-generating element?

- What is the role of photoreactions in providing energy (e.g., perchlorates on Mars' surface, i.e., synergies vs. competition between reactions)?

- How critical is the interaction between the biological and mineralogical part of the ecosystem?

- How critical is the role of biogenic activity? 
Sub-objective 2. Atmospheric context. Achieving this subobjective would require the following question to be addressed:

- What is the expected range of atmospheric biosignatures that arise as redox pairs under differing exoplanetary conditions?

Key Objective 3. To follow the data: Evaluate the potential for life in different planetary environments (from microscale to planets). In general, databases in the context of astrobiology, and for biosignatures in particular, have wide-ranging formats-ranging from images, spectra, composition, and so on. It is a general challenge to bring together the various communities into one centrally defined biosignature database and to conceptualize the necessary tools related to, for example, data interpretation and crosscorrelation in time and space.

Exoplanets-There will be a revolution in exoplanetary science in the next 20 years. We are entering a golden age where first rocky-planet characterizations (age, bulk density, and first atmospheric spectra) for a statistical sample of (hot or/and warm) super-Earths will become available via European-led observations (PLATO 2.0, E-ELT, etc.). Critically, however, the first data to be received (the "easiest targets") will likely be

(a) Hot super-Earths with thick (hundreds of bar) atmospheres of lighter species, such as $\mathrm{H}_{2}$ : Hot planets orbit their stars quicker and hence deliver more data over a given time period. Light atmospheres are more extensive than heavy atmospheres, so they give stronger signals.

(b) Warm super-Earths orbiting in the habitable zone of cool stars such as M dwarfs (see, e.g., Tarter et al. 2007): Here, the flux from the planet is easier to detect when it orbits a dimmer star. Also, since the habitable zones lie closer to the star, the planet orbits faster; hence more data can be collected over a given time period.

To interpret data from (a) and (b), Europe's astrobiology community of biologists, geologists, and atmospheric scientists will need to come together to "follow the data" and ask the question: Can life exist on such worlds?

Several studies have investigated possible photosynthesis on worlds orbiting foreign stars. Success will come from researchers who currently focus on the Earth sciences and who can adapt to a wider, nonterrestrial environmental context. This is currently occurring much faster in the United States than in Europe. Bains et al. (2014) investigated photosynthesis in $\mathrm{H}_{2}$ atmospheres. Kiang et al. (2007) investigated potential photosynthesis on planets orbiting, for example, cool M stars. Haas (2010) hypothesized the possibility of chlorine-based photosynthesis in an exoplanetary context. Cockell et al. (2009) investigated the possibility of photosynthesis on planets orbiting main-sequence stars.

Issues that need to be further discussed include the following: Can life evolve in a thick $\mathrm{H}_{2}$ atmosphere? Can a "photosynthesis-like" process develop on worlds where the energy input of the star peaks at low energy wavelengths? Can life develop on a world with a weak magnetic field or without active plate tectonics?

Sub-objective 1. Super-Earths-role of atmospheric composition. The following questions need to be addressed:
- Do super-Earths retain their primary $\left(\mathrm{H}_{2}\right)$ atmospheres, or are they lost via escape processes?

- What are the climate conditions on such worlds, and what is the relevant photochemistry affecting biosignatures?

- Can photosynthesis develop on such worlds?

- Does the larger planetary mass (up to 10 Earth masses) favor or disfavor plate tectonics, and what is the effect on mineralogy and the development of potential life?

- What are the potential atmospheric biosignatures?

Sub-objective 2. Super-Earths orbiting cooler stars (M $d w a r f s$ and $K d w a r f s$ ). The following questions need to be addressed:

- Are such worlds habitable?

- Do they have sufficient water?

- What is the magnetic protection?

- What is the effect of being tidally locked (i.e., with constant night and day sides)?

- What is their climate, and are there potential biosignatures?

Sub-objective 3. Colocate stratigraphy images with the spectral data. A key challenge is to colocate stratigraphy images of outcrops or sedimentary layered features with the spectral data of the image's relevant features.

Key Objective 4. To follow biosignatures with time: Reach a better understanding of the evolution and preservation of biosignature assemblages with time. Biosignature assemblages operate over wide scales - both in time and from molecular to planetary scales. The timeframe context of life has various key aspects, for example, establishment, evolution, transformation (or death), preservation and/or decay of organisms, as well as the atmospheric (composition, climate) evolutionary aspects and the impact on the associated biosignatures.

On the molecular to cellular scale, biosignature assemblages are a product of the communities of living organisms that produce them. The assemblages of biosignatures for life on early anaerobic Earth are different from those of presentday aerobic Earth, and this difference is related to the evolution of life-forms. The former are not detectable remotely, that is, on an exoplanet, whereas the latter could be. Thus, the longevity and stability of habitable conditions on another planet could influence the kind of life-forms to be expected and their eventual biosignatures.

The preservation of these biosignature assemblages through geological time will be influenced by short- (diagenetic) to long-term physicochemical changes affecting the rocks in which they occur.

Even on much shorter timescales, life-related species, such as $\mathrm{O}_{3}$ and $\mathrm{CH}_{4}$ on modern Earth, display temporal variability. This suggests that long-term modern-day monitoring and searching for biosignatures (e.g., in Mars' and exoplanetary atmospheres) could be advantageous. On the planetary scale, atmospheres and their biosignatures evolve with time due to changes in, for example, biomass emissions, outgassing, escape, and stellar input.

\subsection{Approach to achieve the key objectives}

Due to the far-reaching spatial scales (from the submicron up to the planetary scale), which are inherent to the nature 
of this topic, the scientific approach needs to be both experimental and observational, with a strong modeling foundation.

Dedicated techniques and tools to deal with the multiscale approach from the molecular to the planetary scale are required. In situ science will benefit from promoting the development of new instruments with joint cooperation between science and technology. Also, sample return will be an important part of the search for traces of life, as opposed to traces of abiotic features.

Next ten years. Solar System exploration. A comprehensive review of the known biosignature assemblages through geological time and the evolution of life would be a good starting point in the achievement of these goals. In addition, it will be necessary to investigate biosignature preservation over long geological timescales, in particular, the effects of the different thermal, mechanical, chemical processes on the degradation process.

Actions to be taken to reach the objective should include

- Study of biosignature assemblages on Earth through geological time and different stages of the evolution of life via direct observation in rocks and through experimental approaches;

- On a planetary scale, acquire better knowledge of the time evolution of parameters affecting biosignatures, for example, the UV output of the star, the evolution of planetary climate and photochemistry affecting biosignatures, and so on;

- Technical improvement permitting in situ characterization of biosignatures at the microbial scale, evaluation of the different degradation factors, and accurate estimation of the maturity degree of the organic matter.

What is also missing is a comprehensive review of known and potential metabolic redox couples, taking into account the fact that microorganisms are extremely adaptive to new possible and parallel uses of other pathways. It is desirable to expand both technological capability and research infrastructure. This aspect can be addressed by better interaction between the geological and biological communities.

Actions to be taken to reach the objective should include

- Networking in observational and modeling centers in contact (in situ) science;

- Research activities (experimental approaches in the laboratory);

- Studies of redox couples in the (extra)terrestrial environments (ancient and modern) and Earth-analog activities (e.g., early Earth);

- Introducing interdisciplinary bio-geo courses at the undergraduate level.

Exoplanetary research. For exoplanets, we need better constraints for models that predict biosignature spectra. These include better knowledge of chemical reaction rates, climate databases, and construction of interdisciplinary databases with the common goal of biosignature detection.

Atmospheric chemical reactions are currently not well measured at high temperatures and in different atmospheric compositions. More scientific proposals are needed, and more infrastructure facilities are needed in Europe to better constrain these values.
The effect of clouds upon the observed atmospheric spectra is currently not known. Current observations suggest many hot super-Earths have clouds. Understanding this is a challenging task requiring more postdoctoral staff and experienced personnel in Europe. Cloud formation is a 3-D processes, and we need teams that can better build the next generation of 3-D models, including cloud formation for a wide range of exoplanetary atmospheres and their impact upon the width of the habitable zone.

Ten to twenty years from now. Solar System exploration. Ten years from now, we should have received the first samples collected on Mars, hopefully with, at a minimum, traces of organic molecules and, at best, traces of martian life. In the first place, adequate curation facilities will be necessary for which it is imperative to start now. Also, the small size and limited quantity of material available for analysis will place constraints on instrumentation. It will be necessary to develop, starting now, more sophisticated instrumentation with spatial and quantification resolutions significantly higher than those presently available. Moreover, these instruments will need to be developed so that it is possible to analyze samples in containment, that is, a hermetically sealed container, because of planetary protection considerations.

Planetary exploration from the astrobiological point of view should already have extended to icy moons with the ESA mission JUICE to Jupiter and Europa and the NASA Multiple Flyby mission to Europa (formerly Europa Clipper) being developed and due to launch in the next decade. These missions will test our ability to detect traces of life (if it exists) from atmospheric signatures. Ground preparations in terms of experiments and instrumentation to improve these capabilities need to be undertaken.

Exoplanetary research. Atmospheric science needs to expand on existing theoretical modeling studies in order to distinguish signals of planets with life from those without and, hence, calculate theoretical spectra to compare with forthcoming observational data. This will pave the way to interpret the data from the European missions PLATO 2.0 and E-ELT in the next 20 years.

After twenty years. Solar System exploration. Astrobiological research in the Solar System presents two major long-term challenges. The first continues to be the search for life on Mars. It is possible that the first rocks returned will either not contain traces of life (the in situ instrumentation used to choose the rocks, although highly ingenious, is limited with respect to the laboratory instrumentation used on the ground) or will contain signatures that remain ambiguous, despite the sophistication of the ground instrumentation used in their analysis. A second lot of returned samples will be necessary either to confirm or refute hypotheses based on the first batch of samples, or simply to continue the search. Another challenge for Mars is the possibility of "deep" drilling (tens to hundreds of meters) into the surface. The rationale for drilling is that, if life still exists on the planet, it will be in some warm hydrothermal niche or preserved in the cryosphere. Moreover, drilling will provide access to older horizons that may be better preserved and may therefore contain better-preserved biosignatures. It is 
these deep-drilled samples that may furnish material for the second or third batch of returned samples.

The question of how to deal with (storage and curation) and how to analyze and detect martian life in samples containing potential extant life needs to be addressed shortly. Even though the first batch of returned samples will address past life and fossilized biosignatures, we cannot be $100 \%$ sure that the samples will not contain extant cells.

The second major challenge will be sample return from an icy moon, especially if the flybys provide tantalizing indications of habitable conditions and the presence of complex organics. Great efforts will be necessary to prepare for such missions and for the curation of the returned samples. Their analysis will pose additional instrumental and technological challenges.

Exoplanetary research. We will have likely identified a wealth of new exoplanets-from hot gas planets down to cool rocky planets in the habitable zone. Their ages and mean densities will be characterized, and there will likely be first atmospheric spectra for hot and warm super-Earths. Figure 7 gives an integrated view of Research Topic 5 together with its timeline.

\subsection{European strengths and needs}

Europe already has the expertise in different disciplines (chemistry, geology, prebiotic chemistry, biology, etc.) and laboratories for the above studies to be carried out. Present and planned European missions in the Solar System such as Mars Express, Venus Express, ExoMars, and JUICE have expanded, and will continue to expand, our knowledge of habitability in an astrobiological context.

In particular, the European-Russian-led mission ExoMars, which will build on knowledge gained during the Mars Express mission, has the unique capability to obtain subsurface samples that are critical for contact science. The combination of optical microscopy with spectroscopic techniques, such as IR and Raman, is also a main strength of this mission together with its planned capabilities of detailed organic identification. Hence, ExoMars will potentially deliver unique subsurface data on Mars.

European missions, such as PLATO 2.0 (planned 2024), will for the first time deliver bulk composition and accurate ages for rocky exoplanets in the habitable zone. E-ELT (planned $\sim 2025$ ) could possibly deliver the first exoplanetary atmospheric biosignatures. In addition to PLATO 2.0 and E-ELT, the German-Spanish CARMENES program will detect exo-Earths in the habitable zone of M dwarfs. Europe has gathered considerable expertise in managing comprehensive databases from previous, ongoing, and planned European missions in the Solar System.

Europe also features state-of-the-art research centers for numerical modeling - both in an atmospheric and in a wet chemistry context-which are already established, for

\begin{tabular}{|c|c|c|}
\hline \multicolumn{3}{|c|}{$\begin{array}{c}\text { Research Topic 5 - } \\
\text { Biosignatures as Facilitating Life Detection }\end{array}$} \\
\hline \multicolumn{3}{|l|}{ KO 1: To distinguish life from non-life } \\
\hline \multirow{2}{*}{ Advanced detection technologies: } & \multicolumn{2}{|l|}{ High resolution methods (remote \& in situ) } \\
\hline & Biosignature !̣dentification & \\
\hline \multirow{2}{*}{ Modelling } & Signal / environment characterisation & \\
\hline & Characteristics of life vs. non-life signals & \\
\hline \multicolumn{3}{|c|}{ KO 2: To follow the energy: Identify energy sources, rẹdox couples and photoreactions } \\
\hline \multirow{2}{*}{ Field \& laboratory studies } & Redox couples in terrestrial environments & \\
\hline & List of ancient/modern; metabolic redox couples & \\
\hline \multicolumn{3}{|c|}{ KO 3: To follow the data: Evaluate the potential for life in different planetary environments } \\
\hline \multirow[t]{2}{*}{ Observations from space } & \multicolumn{2}{|l|}{ JWST, PLATO 2.0} \\
\hline & \multicolumn{2}{|l|}{ Age of rocky planets in habitable zdnes } \\
\hline \multirow[t]{2}{*}{ Observations from ground } & \multirow{2}{*}{\multicolumn{2}{|c|}{$\begin{array}{c}\text { E-ELT } \\
\text { Atmospheric biosignaturés from exoplanets }\end{array}$}} \\
\hline & & \\
\hline Solar system missions & \multicolumn{2}{|c|}{ ExoMars, JUICE, icy moons: fly-bys, landers, sample returns, curation } \\
\hline \multicolumn{3}{|c|}{ KO 4: To follow biosignatures with time } \\
\hline \multirow[t]{2}{*}{ Field studies } & \multicolumn{2}{|c|}{ Biosignatures on Earth: variation through geological times } \\
\hline & $\vdots$ & 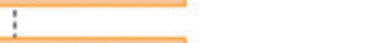 \\
\hline Laboratory studies & \multicolumn{2}{|c|}{ Effects of environment on preservation of biosignatures } \\
\hline
\end{tabular}

FIG. 7. AstRoMap Roadmap, approaches to reach the key objectives of Research Topic 5 "Biosignatures as Facilitating Life Detection" within the next 10, 20, or follow-on years. 
example, in DLR in Germany, CNRS in France, IWF in Austria, CSIC in Spain. There also exist in Europe very well-established teams working on the geological/geochemical context of energy for life. These centers can seed future research.

Furthermore, Europe already has key expertise that can seed understanding of biosignature assemblages through geological time and during the evolution of life, as well as for experimentally testing biosignature preservation. European exoplanetary missions like PLATO 2.0 will revolutionize these issues by delivering planetary ages and bulk composition of Earth-like planets with unprecedented accuracy. This, coupled with data from E-ELT, will revolutionize exoplanetary science.

\section{Conclusions and Recommendations}

\subsection{Cross-cutting issues of relevance}

8.1.1. Biotechnology. The research of extremophiles has not only provided us with interesting insights into the origin and evolution of life on Earth, it has also initiated a broad range of useful products and applications within academic research, biotechnology, the medical field, and industry (see, e.g., Anitori, 2012; Singh, 2013). The reason for this is that extremophiles can withstand harsh conditions, and they provide a range of unique innovative products and sustainable procedures. Studying every category of extremophile (thermophiles, psychrophiles, halophiles, acidophiles, alkaliphiles, piezophiles, etc.) has proven to be highly useful, and research on the biotechnology of extremophiles is thus a rapidly growing field. Examples of useful products range from enzymes important to research (e.g., Taq polymerase for the polymerase chain reaction) and industry, to compounds for medicine (antibiotics, vitamins, antioxidants), food (flavor enhancer, feed supplement, natural dyes, preservative), industrial chemicals, cosmetics (e.g., compatible solutes from halophiles as protection against UV radiation), and different types of novel concepts for diagnostics. Furthermore, in recent years a whole range of novel, sustainable procedures have been developed with the aim to solve today's environmental and energy problems, such as biopolymers for the production of plastics, environmentally friendly biofuels, bioremediation of hazardous compounds (oil industry, heavy metals to hazardous chemicals), counteraction of the greenhouse effect, and making agriculturally relevant plants more resistant to high salt concentrations, heavy metal contaminations, or drought. Last, but not least, some extremophiles have also proved to be useful for sustainable mining not only on Earth but possibly in space, such as on asteroids (e.g., Cockell, 2010). Due to all these possibilities that allow industry to reduce energy use, employ sustainable processes, and develop novel energy sources, products, and procedures, many countries worldwide are now investing large amounts of money to support this development, applied to endeavors on Earth and in space (e.g., the German biotechnology program of BMBF-https://www.biotechnologie2020plus.de; http://www.planetaryresources.com). Essential tasks in this mission include bioprospecting, that is, the screening of novel extremophiles in a variety of extreme ecosystems; how to support appropriate fundamental research (e.g., molecular biology, biochemistry, genetics); and how to avoid the pitfalls along the path towards biotechnological inventions (e.g., CAREX, 2011b).

8.1.2. International cooperation. International collaboration in space activities in recent decades has enabled several spectacularly successful missions. One lesson learned from these years of cooperation is that international agreements have the capacity to improve the feasibility of joint programs, as shown, for instance, in the case of the ISS, the Solar and Heliospheric Observatory (SOHO), the Cassini-Huygens missions, or ALMA. Although international cooperation tends to strengthen missions and program stability, the case of ExoMars illustrates that it can also be a challenging avenue, as was the case in 2012 when NASA withdrew its major contribution to the program, which threatened its viability and required ESA to change its programmatic approach and develop new partnerships.

Apart from the fact that astrobiology is an issue of global interest by essence, considering the nature of the means and infrastructures required to perform astrobiology studies and investigations as well as geographical considerations, international cooperation has become a prerequisite for all actors.

Space science missions related to, or dedicated to, astrobiology are more and more complex and involve higher budgets. Their increased complexity requires pooling and coordinating scientific and technical skills and competencies that are sometimes unique around the globe. This is the case in the context of, for example, modeling of the origin of planetary systems, the characterization of exoplanets, or the design and development of scientific instruments dedicated to the detection of extant or extinct life on planetary (or icy) bodies. As scientific ambitions involve many instrumentation and technology challenges, the cost of missions is naturally impacted, sometimes to the point that they become unaffordable for one single agency (e.g., a Mars sample return mission). When possible, well-planned and wellimplemented international cooperation allows the costs to be split and the risks to be spread.

International cooperation in space missions related to astrobiology is indeed not something new. Over the past decades, many of these endeavors involved strong collaboration between scientists from different regions of the world. Of the 10 scientific instruments on board NASA's Mars Science Laboratory, two were provided by international partners [Centro de Astrobiología (Spain) and Russian Academy of Sciences Space Research Institute], and three involved international cooperation (two from Europe and one from Canada). On the European side, the ExoMars program also involves international cooperation with Russia and some participation from the United States for both elements of the mission (2016 orbiter and 2018 rover). As to Mars exploration in the longer term, and with the caching capability of NASA's Mars2020 rover to be launched in 2020, the next major challenge lies in the return of samples from the Red Planet; this considerable effort will undoubtedly require strong international cooperation. 
In addition to collaboration with historic partners (United States, Russia, Japan) and with the ambitious plans of other countries, Europe and European countries should consider strengthening scientific collaboration with India (the Indian Space Research Organisation's Mars Orbiter Mission has been orbiting Mars since September 2014) and China, which is investing large budgets in the development of a planetary exploration program and in detection and characterization of exoplanets. Considering the very strong expertise of the European community in space exposure experiments, the planned retirement of the ISS in 2024 will also require new and enhanced partnerships with the Chinese human space program to be considered.

Astrobiology can involve the design and implementation of complex and costly space missions and ground infrastructures, but investigations in this domain can also start with equipment as simple as sampling kits, a camera, a GPS, and a pair of shoes. Many locations on Earth present some environmental conditions that are analogous to those of extraterrestrial bodies, while others preserve records of the earliest life-forms. Studying the origin of life and the presence of past or present life in space is a strongly attractive scientific subject, with multidisciplinary aspects that can easily attract the interest of scientists and stakeholders of (emerging) countries, acting as stimulus to invest in space research and exploration. Exploring and exploiting these sites allows scientific questions of relevance to life on Earth and beyond (e.g., early Earth, origin of life, biogeochemical cycles) to be addressed. These sites are spread over the planet on every continent; some are easily accessible, and others require heavy logistics and complicated planning. Performing investigations in these analogue sites represents a unique opportunity to create interactions and collaboration at the academic level. In 2011, a COSPAR workshop report concluded that "Earth-based field research can contribute to our knowledge concerning planetary protection, astrobiology, geology, robotic missions, instrument testing, technology testing, human exploration, management approaches, international cooperation, student involvement, and public outreach" (Ehrenfreund et al., 2011). This workshop suggested the concept of an international program dedicated to research activities performed in analog study sites. It specifically highlighted that such activities allow for space science issues to be tackled at a relatively low cost, facilitating the involvement of scientists from the (developing) countries in which these sites are located; this is particularly relevant as local universities and research groups often have detailed knowledge of these environments and their ecosystem. Such a delocalized approach is also relevant to the development of observation networks (e.g., fireball networks for observation of bolides and retrieval of meteorites; telescopes in remote dark areas).

The European scientific community has strong assets and experience in developing and maintaining collaborations with non-European teams. In addition to collaborations in the context of missions and programs implemented by space agencies and research organizations, the community has developed bottom-up platforms and initiatives that allow effective interactions. EANA is associated with international astrobiology groups in Brazil, China, Japan, Mexico, and the United States; representatives from these countries have privileged access to European astrobiologists, and the annual EANA workshop is the occasion to develop and nurture fruitful collaborations. Another good example of an efficient mechanism to expand the geographical scope of scientific collaboration can be found in the Europlanet Transnational Access scheme. Under the European Commission Seventh Framework Programme, the project has organized and coordinated competitive access to five field sites of relevance for planetary sciences, including three in non-European countries (Morocco, Tunisia, and Russia). During the upcoming funding period (European Commission Horizon 2020 program), Europlanet will organize competitive access to three new planetary field analogues, including one in Ethiopia. Surveying and identifying the good practices defined and implemented by EANA, Europlanet and other similar bottom-up initiatives would allow further joint initiatives between European and non-European teams to be developed and eventually strengthen European astrobiology.

Based on past and current experience and success, Europe should foster its involvement in international space partnerships (Rosa et al., 2013). Through its Horizon 2020 and subsequent programs, the European Union could contribute to facilitating European scientists and industry's involvement in these activities. Prominent candidates for Europe's involvement in international cooperation concerning astrobiology-related fields are as follows:

- A comprehensive robotic Mars exploration program along with development of technological concepts for sample return should be an essential element of a coordinated international space research program in cooperation with major non-European space-faring nations, in which Europe must keep a leading role in its predominant components.

- The exploitation of the ISS and the evolution of the European LEO infrastructure. In parallel, negotiations should be supported with established and emergent space-faring nations, such as China, to open fair opportunities for the continuation and extension of astrobiology research in LEO beyond 2020.

- Exploratory missions to the icy moons of the giant planets, especially Jupiter's moon Europa and Saturn's moons Titan and Enceladus, targeting in situ sample analysis and finally sample return.

- Involvement in major international efforts such as the E-ELT and the American JWST and developing the necessary expertise in order to interpret and understand the new data from such missions and to develop the next generation of models to anticipate this exciting data.

\subsection{Towards a better coordination of astrobiology research in Europe-the need for a pan-European platform}

Although the European astrobiology community has reached a good level of maturity (see Section 1.2.3) and, on a theoretical level, Europe is traditionally strong and has closed, or is closing, the gaps with other space science powers, in particular the United States, it still lags behind on specific topics (e.g., ground-based observations of the outer Solar System). It seems that European competitiveness in this field is weakened by fragmentation.

On a programmatic level, European astrobiology is dispersed and fragmented because it encompasses many scientific 
disciplines and because, in some countries, astrobiology is only marginal (and therefore not a priority). Moreover, the current institutional landscape does not allow pan-European stability, strategic programming, and stable, streamlined financial support. Organizations such as EANA are stable (EANA was set up in 2001). EANA is a grassroots organization (the heart and soul of European astrobiology), but it does not have a programmatic mandate. A platform such as Europlanet, which has also been rather stable over the past decade, is efficient in providing programmatic coordination and infrastructure support, but its mandate and faith rely heavily on periodic competitive funding (at the level of the European Commission) and support that is already available at the national level. ESA provides the means and platform to do science in the medium to long term, but the actual experiments and data exploitation are supported at the national level. This diversity of disciplines and actors-and the resulting complexity-limits Europe's capacity to perform even better in the field of astrobiology. It also impacts the capacity of European astrobiology research to approach public outreach and engagement in a holistic, coherent manner (although initiatives such as the EAC intend to tackle this issue).

Throughout the AstRoMap project, and even more when surveying future perspectives and priorities for European astrobiology, the need for a stable pan-European programmatic platform (possibly virtual) was expressed without ambiguity. Such a platform, enabling and dedicated to astrobiology research, would serve and allow progress to be made on all the scientific objectives presented in this roadmap and would definitely be a strong asset for the European astrobiology community. Such a platform should

- Encourage and capitalize on multidisciplinary research and projects that include diverse space sciences and/or Earth science disciplines;

- Allow a rational, possibly prioritized, access to relevant research infrastructures and equipment, possibly through development of specific networks;

- Allow the implementation of (and mobilize around) a prioritized research plan, taking into consideration the strengths and expertise available in different countries and allowing them to be further improved and shared;

- Provide a platform for the management, access, and archiving of relevant scientific data;

- Facilitate the design and development of new techniques and technologies;

- Provide stable funding and support allowing scientific objectives to be addressed in the medium to long term;

- Catalyze and facilitate undergraduate and graduate education and help with the development of $\mathrm{PhD}$ programs and postdoctoral opportunities;

- Facilitate exchange of scientific and technical staff;

- Represent a European anchor for international relations;

- Represent a focal point for coherent public outreach and engagement.

When considering the current European astrobiology landscape and the number of actors and stakeholders and their diversity, it appears clear that such a platform is challenging to define and design. In this context, and with the intent to design a platform that will help fulfill the expectations and ambitions of its end users, the scientific com- munity, it is critical that heads of main groups, laboratories, and institutes are consulted, or that they organize themselves (possibly through EANA) in such a way as to define the baseline specifications and functions of a European platform for astrobiology. Such specifications would then be proposed and discussed with the national and European research organizations and space agencies. In addition to necessary discussion and consultation, and considering that such a platform will need time to take form, one can already consider that

- It should be science-driven with a strong representation of the community in its governance.

- It should evolve organically, starting with pilot initiatives such as a network of institutes and laboratories, a framework for $\mathrm{PhD}$ students/postdoc exchange, a framework for the use of infrastructures and access to field sites dedicated to collaborative projects, and eventually grow towards a more institutionalized structure, using the experience gained through pilots.

- It should be flexible and able to adapt to the European and international landscape as well as evolving priorities and breakthrough discoveries.

- It should be decentralized as much as possible to reap the benefits of European diversity without involving a heavy administrative burden and overhead costs.

- It should be, as much as possible, in a position to provide end-to-end funding with a common (set of) scheme(s) to avoid the complication of various funding procedures and principles.

In this context, a detailed survey of good practice across European initiatives and projects, as well as international programs [e.g., the UN Committee on the Peaceful Uses of Outer Space (COPUOS) capacity-building programs, the International Ocean Drilling Program (IODP), or the Census of Marine Life] is deemed necessary. It is also strongly recommended that the model applied by the NASA Astrobiology (virtual) Institute be taken into consideration, as this efficient, flexible mechanism has been active since 1998 and has therefore capitalized a lot of experience. There is no doubt that the establishment of a well-designed and managed European Astrobiology Platform (or Institute) would catalyze tremendous scientific achievements in the field and streamline and optimize the use of funding and infrastructure. It is strongly recommended that steps be taken towards the definition and implementation of such a concept.

\section{Acknowledgments}

The AstRoMap team would like to thank the authors of this roadmap as well as additional participants to the AstRoMap workshops:

Roberto Barbieri, Daniela Billi, Charles Cockell, Cristiano Cosmovici, Grégoire Danger, Jean-Pierre de Vera, Enrico Flamini, Frédéric Foucher, Stefan Fox, Javier Gómez-Elvira, Jean-Claude Guillemin, Rodríguez-Manfredi Jose-Antonio, Terence Kee, Kevin Lepot, Harry Letho, Kirsi Letho, Robert Macke, Nigel Mason, Gian Gabriele Ori, Victor Parro, David Pearce, Olga Prieto-Ballesteros, Kepa Ruiz-Mirazo, Henry Strasdeit, Feng Tian, Diego Turrini, Boris Zaprudin.

AstRoMap was supported by European Community's Seventh Framework Programme (FP7/2007-2013) under Grant Agreement n 313102. 
Open access cost of the AstRoMap roadmap is supported by the European Science Foundation (ESF).

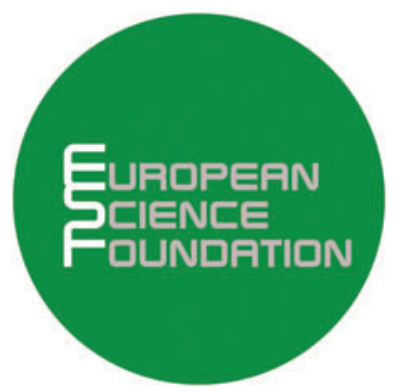

\section{Author Disclosure Statement}

None of the authors have any commercial associations that might create a conflict of interest in connection with this manuscript. No competing financial interests exist.

\section{References}

Ackermann, M. (2013) Microbial individuality in the natural environment. ISME J 7:465-467.

Adhikari, D.-D., Thakuria, T., and Medhi, C. (2000) MCSCF calculations on metal ion $\left(\mathrm{Li}^{+}, \mathrm{Na}^{+}, \mathrm{Be}^{2+}\right)$ affinities of a few carbonyl molecules in the ground and $1,3 \mathrm{n} \pi^{*}$ excited states. Indian J Chem 39:792-801.

Albuquerque, P. and Casadevall, A. (2012) Quorum sensing in fungi-a review. Med Mycol 50:337-345.

Alibert, Y., Mordasini, C., Benz, W., and Winisdoerffe, C. (2005) Models of giant planet formation with migration and disc evolution. Astron Astrophys 434:343-353.

Allodi, M.A., Baragiola, R.A., Baratta, G.A., Barucci, M.A., Blake, G.A., Brucato, J.R., Contreras, C., Cuylle, S.H., Boduch, Ph., Fulvio, D., Gudipati, M.S., Ioppolo, S., Kaňuchová, Z., Lignell, A., Linnartz, H., Palumbo, M.E., Raut, U., Rothard, H., Salama, F., Savchenko, E.V., SciammaO'Brien, E., and Strazzulla, G. (2013) Complementary and emerging techniques for astrophysical ices processed in the laboratory. Space Sci Rev 180:101-175.

ALMA. (2014, November 5) Revolutionary ALMA image reveals planetary genesis. Joint ALMA Observatory, Santiago, Chile. Available online at http://www.almaobservatory.org/press-room/ press-releases/771-revolutionary-alma-image-reveals-planetarygenesis

Altermann, W. and Wotherspoon, J.McD. (1995) The carbonates of the Transvaal and Griqualand West sequences of the Kaapvaal craton, with special reference to the Lime Acres limestone deposit. Miner Depos 30:124-134.

Altwegg, K., Balsiger, H., Bar-Nun, A., Berthelier, J.J., Bieler, A., Bochsler, P., Briois, C., Calmonte, U., Combi, M., De Keyser, J., Eberhardt, P., Fiethe, B., Fuselier, S., Gasc, S., Gombosi, T.I., Hansen, K.C., Hässig, M., Jäckel, A., Kopp, E., Korth, A., LeRoy, L., Mall, U., Marty, B., Mousis, O., Neefs, E., Owen, T., Rème, H., Rubin, M., Sémon, T., Tzou, C.-Y., Waite, H., and Wurz, P. (2015) 67P/ChuryumovGerasimenko, a Jupiter family comet with a high $\mathrm{D} / \mathrm{H}$ ratio. Science 347, doi:10.1126/science.1261952.

Andrianantoandro, E., Basu, S., Karig, D.K., and Weiss, R. (2006) Synthetic biology: new engineering rules for an emerging discipline. Mol Syst Biol 2, doi:10.1038/ msb4100073.

Anitori, R.P. (2012) Extremophiles: Microbiology and Biotechnology, Caister Academic Press, Norfolk, UK.

Arndt, N.T. (1994) Archean komatiites. In Archean Crustal Evolution, edited by K.C. Condie, Elsevier, Amsterdam, pp 11-44.

Arndt, N.T. and Nisbet, E.G. (2012) Processes on the young Earth and the habitats of early life. Annu Rev Earth Planet Sci 40:521-549.

Baaske, P., Weinert, F.M., Duhr, S., Lemke, K.H., Russell, M.J., and Braun, D. (2007) Extreme accummulation of nucleotides in simulated hydrothermal pore systems. Proc Natl Acad Sci USA 104:9346-9351.

Bach, W., Paulick, H., Garrido, C.J., Ildefonse, B., Meurer, W.P., and Humphris, S.E. (2006) Unraveling the sequence of serpentinization reactions: petrography, mineral chemistry, and petrophysics of serpentinites from MAR $15^{\circ} \mathrm{N}$ (ODP Leg 209, Site 1274). Geophys Res Lett 33, doi:10.1029/ 2006GL025681.

Bains, W., Seager, S., and Zsom, A. (2014) Photosynthesis in hydrogen-dominated atmospheres. Life 4:716-744.

Baker, J.A., Bizzarro, M., Wittig, N., Connelly, J.N., and Haack, H. (2005) Early planetesimal melting from an age of 4.5662 Gyr for differentiated meteorites. Nature 436:1127-1131.

Bakermans, C., editor. (2015) Microbial Evolution under Extreme Conditions, Walter de Gruyter $\mathrm{GmbH}$, Berlin.

Barge, L.M., Kee, T.P., Doloboff, I.J., Hampton, J.M., Ismail, M., Pourkashanian, M., Zeytounian, J., Baum, M.M., Moss, J.A., Lin, C.K., Kidd, R.D., and Kanik, I. (2014) The fuel cell model of abiogenesis: a new approach to origin-of-life simulations. Astrobiology 14:254-270.

Baross, J.A. and Hoffman, S.E. (1985) Submarine hydrothermal vents and associated gradient environments as sites for the origin and evolution of life. Orig Life Evol Biosph 15:327-345.

Baross, J.A. and Martin, W.F. (2015) The ribofilm as a concept for life's origin. Cell 162:13-15.

Barrangou, R. and Marraffini, L.A. (2014) CRISPR-Cas systems: prokaryotes upgrade to adaptive immunity. Mol Cell 54:234-244.

Bauermeister, A., Rettberg, P., and Flemming, H.C. (2014) Growth of the acidophilic iron-sulfur bacterium Acidithiobacillus ferrooxidans under Mars-like geochemical conditions. Planet Space Sci 98:205-215.

Bell, E.M. (2012) Life at Extremes: Environments, Organisms, and Strategies for Survival, CAB International, Wallingford, UK.

Bennet, P. and Omelon, C. (2011) Microbial geochemistry: at the intersection of disciplines. In Frontiers in Geochemistry: Contribution of Geochemistry to the Study of the Earth, edited by R. Harmon and A. Parker, Wiley-Blackwell, Chichester, UK.

Bennett, C.J., Pirim, C., and Orlando, T.M. (2013) Spaceweathering of Solar System bodies: a laboratory perspective. Chem Rev 113:9086-9150.

Benzerara, K., Skouri-Panet, F., Li, J., Férard, C., Gugger, M., Laurent, T., Couradeau, E., Ragon, M., Cosmidis, J., Menguy, N., Margaret-Olivera, I., Tavera, R., Lopez-Garcia, P., and Moreira, D. (2014) Intracellular Ca-carbonate biomineralization is widespread in cyanobacteria. Proc Natl Acad Sci USA 111:10933-10938.

Beuther, H., Klessen, R.S., Dullemond, C.P., and Henning, Th., editors. (2014) Protostars and Planets VI, University of Arizona Press, Tucson. 
Billi, D. (2012) Anhydrobiotic rock-inhabiting cyanobacteria: potential for astrobiology and biotechnology. In Adaption of Microbial Life to Environmental Extremes: Novel Research Results and Application, edited by H. Stan-Lotter and S. Fendrihan, Springer, Wien, pp 119-132.

Blöchl, E., Rachel, R., Burggraf, S., Hafenbradl, D., Jannasch, H.W., and Stetter, K.O. (1997) Pyrolobus fumarii, gen. and sp. nov., represents a novel group of archaea, extending the upper temperature limit for life to $113^{\circ} \mathrm{C}$. Extremophiles 1:14-21.

Bohm, D. (1980) Wholeness and the Implicate Order, Routledge \& Kegan Paul, Taylor Francis Group, London.

Brack, A., Fitton, B., and Raulin, F. (1999a) Exobiology in the Solar System \& the Search for Life on Mars, Report from the ESA Exobiology Team Study 1997-1998, ESA SP-1231, ESA Publications Division, ESTEC, Noordwijk, The Netherlands.

Brack, A., Clancy, P., Fitton, B., Hofmann, B., Horneck, G., Kurat, G., Maxwell, J., Ori, G., Pillinger, C., Raulin, F., Thomas, N., and Westall, F. (1999b) An integrated exobiology package for the search for life on Mars. Adv Space Res 23:301-308.

Brock, T.D. and Freeze, H. (1969) Thermus aquaticus gen. n. and sp. n., a nonsporulating extreme thermophile. J Bacteriol 98:289-297.

Buckel, W. and Thauer, R.K. (2013) Energy conservation via electron bifurcating ferredoxin reduction and proton $/ \mathrm{Na}^{+}$ translocating ferredoxin oxidation. Biochim Biophys Acta Bioenergetics 1827:94-113.

Byerly, G.R., Lowe, D.R., and Walsh, M.M. (1986) Stromatolites from the 3,300-3,500 Myr Swaziland Supergroup, Barberton Mountain Land, South Africa. Nature 319:489-491.

Cafferty, B.J., Gállego, I., Chen, M.C., Farley, K.I., Eritja, R., and Hud, N.V. (2013) Efficient self-assembly in water of long noncovalent polymers by nucleobase analogs. J Am Chem Soc 135:2447-2450.

Callahan, M.P., Smith, K.E., Cleaves, H.J., Ruzickad, J., Stern, J., Glavin, D.P., House, C.H., and Dworkin, J.P. (2011) Carbonaceous meteorites contain a wide range of extraterrestrial nucleobases. Proc Natl Acad Sci USA 108:13995-13998.

Capaccioni, F., Coradini, A., Filacchione, G., Erard, S., Arnold, G., Drossart, P., De Sanctis, M.C., Bockelee-Morvan, D., Capria, M.T., Tosi, F., Leyrat, C., Schmitt, B., Quirico, E., Cerroni, P., Mennella, V., Raponi, A., Ciarniello, M., McCord, T., Moroz, L., Palomba, E., Ammannito, E., Barucci, M.A., Bellucci, G., Benkhoff, J., Bibring, J.P., Blanco, A., Blecka, M., Carlson, R., Carsenty, U., Colangeli, L., Combes, M., Combi, M., Crovisier, J., Encrenaz, T., Federico, C., Fink, U., Fonti, S., Ip, W.H., Irwin, P., Jaumann, R., Kuehrt, E., Langevin, Y., Magni, G., Mottola, S., Orofino, V., Palumbo, P., Piccioni, G., Schade, U., Taylor, F., Tiphene, D., Tozzi, G.P., Beck, P., Biver, N., Bonal, L., Combe, J.P., Despan, D., Flamini, E., Fornasier, S., Frigeri, A., Grassi, D., Gudipati, M., Longobardo, A., Markus, K., Merlin, F., Orosei, R., Rinaldi, G., Stephan, K., Cartacci, M., Cicchetti, A., Giuppi, S., Hello, Y., Henry, F., Jacquinod, S., Noschese, R., Peter, G., Politi, R., Reess, J.M., and Semery A. (2015) The organic-rich surface of comet 67P/ Churyumov-Gerasimenko as seen by VIRTIS/Rosetta. Science 347, doi:10.1126/science.aaa0628.

CAREX. (2011a) CAREX Roadmap for Research on Life in Extreme Environment, CAREX Publication no. 9, Coordination Action for Research Activities on Life in Extreme Environments (CAREX), CAREX Project Office, Strasbourg, France. Available online at http://carex-eu.ameos.net/carexroadmap-for-research-on-life-in-extreme-environments.html
CAREX. (2011b, May 9) CAREX forum: biotechnological explorations of extremophiles to valuable products. Coordination Action for Research Activities on Life in Extreme Environments (CAREX), CAREX Project Office, Strasbourg, France. Available online at http://carex-eu.ameos.net/activities/ carex-forum-on-applications.html

Chan, Y., Lacap, D.C., Lau, M.C., Ha, K.Y., Warren-Rhodes, K.A., Cockell, C.S., Cowan, D.A., McKay, C.P., and Pointing, S.B. (2012) Hypolithic microbial communities: between a rock and a hard place. Environ Microbiol 14:2272-2282.

Chapelle, F.H., O’Neill, K., Bradley, P.M., Methé, B.A., Ciufo, S.A., Knobel, L.L., and Lovley, D.R. (2002) A hydrogenbased subsurface microbial community dominated by methanogens. Nature 415:312-315.

Cloud, P.E. (1948) Some problems and patterns of evolution exemplified by fossil invertebrates. Evolution 2:322-350.

Cockell, C.S. (2003) Impossible Extinction, Natural Catastrophes and the Supremacy of the Microbial World, Cambridge University Press, Cambridge, UK.

Cockell, C.S. (2007) Habitability. In Complete Course in Astrobiology, edited by G. Horneck and P. Rettberg, WileyVCH, Weinheim, pp 151-177.

Cockell, C.S. (2010) Geomicrobiology beyond Earth: microbemineral interactions in space exploration and settlement. Trends Microbiol 18:308-314.

Cockell, C.S., Raven, J.A., Kaltenegger, L., and Logan, R.C. (2009) Planetary targets in the search for extrasolar oxygenic photosynthesis. Plant Ecol Divers 2:207-219.

Cockell, C.S., Balme, M., Bridges, J.C., Davila, A., and Schwenzer, S.P. (2012) Uninhabited habitats on Mars. Icarus 217:184-193.

Cook, A.M., Mattioda, A.L., Ricco, A.J., Quinn, R.C., Elsaesser, A., Ehrenfreund, P., Ricca, A., Jones, N.C., and Hoffmann, S.V. (2014) The Organism/Organic Exposure to Orbital Stresses (O/OREOS) satellite: radiation exposure in low-Earth orbit and supporting laboratory studies of iron tetraphenylporphyrin chloride. Astrobiology 14:87-101.

Cornet, T., Cordier, D., Le Bahers, T., Bourgeois, O., Fleurant, C., Le Mouélic, S., and Altobelli, N. (2015) Dissolution on Titan and on Earth: toward the age of Titan's karstic landscapes. J Geophys Res Planets 120, doi:10.1002/2014JE004738.

Dalrymple, G.B. (2001) The age of the Earth in the twentieth century: a problem (mostly) solved. Geol Soc Spec Publ 190:205-221.

De Duve, C. (1996) Vital Dust: Life as a Cosmic Imperative, Basic Books, New York.

de Vera, J.-P., Möhlmann, D., Butina, F., Lorek, A., Wernecke, R., and Ott, S. (2010) Survival potential and photosynthetic activity of lichens under Mars-like conditions: a laboratory study. Astrobiology 10:215-227.

Dhuime, B., Wuestefeld, A., and Hawkesworth, C.J. (2015) Emergence of modern continental crust about 3 billion years ago. Nat Geosci 8:552-555.

Di Mauro, E., Šponer, J.E., Šponer, J., Giorgi, A., Di Mauro, E., Pino, S., and Costanzo, G. (2015) Untemplated nonenzymatic polymerization of $3^{\prime}, 5^{\prime} \mathrm{cGMP}$ : a plausible route to $3^{\prime}, 5^{\prime}$-linked oligonucleotides in primordia. J Phys Chem B 119:29792989.

Dutrey, A., Semenov, D., Chapillon, E., Gorti, U., Guilloteau, S., Hersant, F., Hogerheijde, M., Hughes, M., Meeus, G., Nomura, H., Piétu, V., Qi, C., and Wakelam, V. (2014) Physical and chemical structure of planet-forming disks probed by millimeter observations and modeling. In Protostars \& Planets VI, edited by H. Beuther, R.S. Klessen, C.P. Dullemond, and Th. Henning, University of Arizona Press, Tucson, pp 317-339. 
Ehrenfreund, P., McKay, C., Walter, N., Rummel, J., and Smith, T., editors. (2011) International Earth-Based Research Program as a Stepping Stone for Global Space ExplorationEarth-X, Committee On Space Research (COSPAR), Paris. Available online at http://www.gwu.edu/ spi/assets/docs/ EarthX_COSPAR2011.pdf

Eigen, M. (1971) Self organization of matter and the evolution of biological macromolecules. Naturwissenschaften 58:465-523.

Elkins-Tanton, L.T. (2012) Magma oceans in the inner Solar System. Annu Rev Earth Planet Sci 40:113-139.

Elmore, S. (2007) Apoptosis: a review of programmed cell death. Toxicol Pathol 35:495-516.

Eriksson, P.G. and Altermann, W. (1998) An overview of the geology of the Transvaal Supergroup dolomites. Environ Geol 36:179-188.

ESA. (2005) Cosmic Vision, Space Science for Europe 2015 2025, ESA BR-247, ESA Publications Division, ESTEC, Noordwijk, The Netherlands. Available online at http:// www.esa.int/esapub/br/br247/br247.pdf

Etiope, G., Schoell, M., and Hosgormez, H. (2011) Abiotic methane flux from the Chimaera seep and Tekirova ophiolites (Turkey): understanding gas exhalation from low temperature serpentinization and implications for Mars. Earth Planet Sci Lett 310:96-104.

EXPOSE-E. (2012) Special collection: the EXPOSE-E mission. Astrobiology 12:373-528.

EXPOSE-R. (2015) Special issue: EXPOSE-R. International Journal of Astrobiology 14:1-142.

Ferus, M., Michalcikovà, R., Shestivskà, V., Šponer, J., and Šponer, J.-E. (2014) High energy chemistry of formamide: a simpler way for nucleobase formation. J Phys Chem A 118:719-736.

Feulner, G. (2012) The faint young Sun problem. Rev Geophys 50, doi:10.1029/2011RG000375.

Fisher, A.T. (2005) Marine hydrogeology: recent accomplishments and future opportunities. Hydrogeol J 13:69-97.

Flock, M., Ruge, J.P., Dzyurkevich, N., Henning, Th., Klahr, H., and Wolf, S. (2015) Gaps, rings, and non-axisymmetric structures in protoplanetary disks: from simulations to ALMA observations. Astron Astrophys 574, doi:10.1051/0004-6361/201424693.

Formisano, V., Atreya, S., Encrenaz, T., Ignatiev, N., and Giuranna, M. (2004) Detection of methane in the atmosphere of Mars. Science 306:1758-1761.

Fornaro, T., Brucato, J.R., Pace, E., Guidi, M.C., Branciamore, S., and Pucci, A. (2013) Infrared spectral investigations of UV irradiated nucleobases adsorbed on mineral surfaces. Icarus 226:1068-1085.

Freissinet, C., Glavin, D.P., Mahaffy, P.R., Miller, K.E., Eigenbrode, J.L., Summons, R.E., Brunner, A.E., Buch, A., Szopa, C., Archer, P.D., Jr., Franz, H.B., Atreya, S.K., Brinckerhoff, W.B., Cabane, M., Coll, P., Conrad, P.G., Des Marais, D.J., Dworkin, J.P., Fairén, A.G., François, P., Grotzinger, J.P., Kashyap, S., ten Kate, I.L., Leshin, L.A., Malespin, C.A., Martin, M.G., MartinTorres, F.J., McAdam, A.C., Ming, D.W., Navarro-González, R., Pavlov, A.A., Prats, B.D., Squyres, S.W., Steele, A., Stern, J.C., Sumner, D.Y., Sutter, B., Zorzano, M.-P., the MSL Science Team. (2015) Organic molecules in the Sheepbed Mudstone, Gale Crater, Mars. J Geophys Res Planets 120:495-514.

Fuchs, G. (2011) Alternative pathways of carbon dioxide fixation: insights into the early evolution of life? Аnпи Rev Microbiol 65:631-658.

Gaillard, F. and Scaillet, B. (2014) A theoretical framework for volcanic degassing chemistry in a comparative planetology perspective and implications for planetary atmospheres. Earth Planet Sci Lett 403:307-316.
García-Ruiz，J.M. (2009) Morphogenesis of self-assembled nanocrystalline materials of barium carbonate and silica. Science 323, doi:10.1126/science.1165349.

Gershenson, C. and Lenaerts, T. (2008) Evolution of complexity. Artif Life 14:241-243.

Goldreich, P. and Ward, W. (1973) The formation of planetesimals. Astrophys $J$ 183:1051-1062.

Gonzalez, G. (2001) The galactic habitable zone: galactic chemical evolution. Icarus 152:185-200.

Gough, D.O. (1981) Solar interior structure and luminosity variations. Sol Phys 74:21-34.

Grenfell, J.L., Gebauer, S., von Paris, P., Godolt, M., and Rauer, H. (2014) Sensitivity of biosignatures on Earth-like planets orbiting in the habitable zone of cool M-dwarf stars to varying stellar UV radiation and surface biomass emissions. Planet Space Sci 98:66-76.

Griffith, R.L., Wright, J.T., Maldonado, J., Povich, M.S., Sigurdsson, S., and Mullan, B. (2015) The G infrared search for extraterrestrial civilisations with large energy supplies. III. The reddest extended sources in WISE. Astrophys J Suppl Ser 217, doi:10.1088/0067-0049/217/2/25.

Grosberg, R.K. and Strathmann, R.R. (2008) The evolution of multicellularity: a minor major transition? Annu Rev Ecol Evol Syst 38:621-654.

Grotzinger, J.P., Sumner, D.Y., Kah, L.C., Stack, K., Gupta, S., Edgar, L., Rubin, D., Lewis, K., Schieber, J., Mangold, N., Milliken, R., Conrad, P.G., Des Marais, D., Farmer, J., Siebach, K., Calef, F., Hurowitz, J., McLennan, S.M., Ming, D., Vaniman, D., Crisp, J., Vasavada, A., Edgett, K.S., Malin, M., Blake, D., Geliert, R., Mahaffy, P., Wiens, R.C., Maurice, S., Grant, J.A., Wilson, S., Anderson, R.C., Beegle, L., Arvidson, R., Hallet, B., Sletten, R.S., Rice, M., Bell, J., Griffes, J., Ehlmann, B., Anderson, R.B., Bristow, T.F., Dietrich, W.E., Dromart, G., Eigenbrode, J., Fraeman, A., Hardgrove, C., Herkenhoff, K., Jandura, L., Kocurek, G., Lee, S., Leshin, L.A., Leveille, R., Limonadi, D., Maki, J., McCloskey, S., Meyer, M., Minitti, M., Newsom, H., Oehler, D., Okon, A., Palucis, M., Parker, T., Rowland, S., Schmidt, M., Squyres, S., Steele, A., Stolper, E., Summons, R., Treiman, A., Williams, R., Yingst, A., and the MSL Science Team. (2014) A habitable fluvio-lacustrine environment at Yellowknife Bay, Gale Crater, Mars. Science 343:1-14.

Haas, J.R. (2010) The potential feasibility of chlorinic photosynthesis on exoplanets. Astrobiology 10:953-963.

Hand, K.P. and Carlson, R.W. (2015) Europa's surface color suggests an ocean rich with sodium chloride. Geophys Res Lett 42, doi:10.1002/2015GL063559.

Hansen, C.J., Esposito, L., Stewart, A.I.F., Colwell, J., Hendrix, A., Pryor, W., Shemansky, D., and West, R. (2006) Enceladus' water vapor plume. Science 311:1422-1425.

Harrison, J.P., Gheeraert, N., Tsigelnitskiy, D., and Cockell, C.S. (2013) The limits for life under multiple extremes. Trends Microbiol 21:204-212.

Harrison, J.P., Dobinson, L., Freeman, K., McKenzie, R., Wyllie, D., Nixon, S.L., and Cockell, C.S. (2015) Aerobically respiring prokaryotic strains exhibit a broader temperature$\mathrm{pH}-$ salinity space for cell division than anaerobically respiring and fermentative strains. $J R$ Soc Interface 12, doi:10.1098/rsif.2015.0658.

Hartmann, W.K. and Davis, D.R. (1975) Satellite-sized planetesimals and lunar origin. Icarus 24:504-515.

Hecht, M.H., Kounaves, S.P., Quinn, R.C., West, S.J., Young, S.M.M., Ming, D.W., Catling, D.C., Clark, B.C., Boynton, W.V., Hoffman, J., DeFlores, L.P., Gospodinova, K., Kapit, J., and Smith, P.H. (2009) Detection of perchlorate and the soluble 
chemistry of martian soil at the Phoenix lander site. Science 325:64-67.

Herschy, B., Whicher, A., Camprubi, E., Watson, C., Dartnell, L., Ward, J., Evans, J.R.G., and Lane, N. (2014) An origin-oflife reactor to simulate alkaline hydrothermal vents. $J \mathrm{Mol}$ Evol 79:213-227.

Herzberg, C., Asimow, P.D., Arndt, N., Niu, Y., Lesher, C.M., Fitton, J.G., Cheadle, M.J., and Saunders, A.D. (2007) Temperatures in ambient mantle and plumes: constraints from basalts, picrites, and komatiites. Geochem Geophys Geosyst 8:1-34.

HISPAC. (2013) Towards a Science-Led One ESA, ESA BR315, ESA Publications Division, ESTEC, Noordwijk, The Netherlands. Available at http://www.esa.int/About_Us/ESA_ Publications/ESA_Publications_Brochures/ESA_BR-315_ Towards_a_Science-led_One_ESA

Hoehler, T.M. (2007) An energy balance concept for habitability. Astrobiology 7:824-838.

Hoehler, T.M. and Westall, F. (2010) Mars Exploration Program Analysis Group Goal One: determine if life ever arose on Mars. Astrobiology 10:859-867.

Hofmann, A. and Harris, C. (2008) Stratiform alteration zones in the Barberton greenstone belt: a window into subseafloor processes 3.5-3.3 Ga ago. Chem Geol 257:224-242.

Hofmann, H.J., Grey, K., Hickman, A.H., and Thorpe, R.I. (1999) Origin of $3.45 \mathrm{Ga}$ coniform stromatolites in Warrawoona Group, Western Australia. Geol Soc Am Bull 111:1256-1262.

Holland, H.D. (1984) The Chemical Evolution of the Atmosphere and Oceans, Princeton University Press, Princeton, NJ.

Holland, H.D. (2006) The oxygenation of the atmosphere and oceans. Philos Trans $R$ Soc Lond B Biol Sci 361:903-915.

Holm, N.G., Oze, C., Mousis, O., Waite, J.H., and GuilbertLepoutre, A. (2015) Serpentinization and the formation of $\mathrm{H}_{2}$ and $\mathrm{CH}_{4}$ on celestial bodies (planets, moons, comets). Astrobiology 15:587-600.

Hordijk, W., Hein, J., and Steel, M. (2010) Autocatalytic sets and the origin of life. Entropy 12:1733-1742.

Horneck, G. (1993) Responses of Bacillus subtilis spores to space environment: results from experiments in space. Orig Life Evol Biosph 23:37-52.

Horneck, G. (2000) The microbial world and the case for Mars. Planet Space Sci 48:1053-1063.

Horneck, G. and Rettberg, P., editors. (2007) Complete Course in Astrobiology, Wiley-VCH, Berlin.

Horneck, G., Klaus, D.M., and Mancinelli, R.L. (2010) Space microbiology. Microbiol Mol Biol Rev 74:121-156.

Horneck, G., Rettberg, P., Walter, N., and Gomez, F. (2015) European landscape in astrobiology, results of the AstRoMap consultation. Acta Astronaut 110:145-154.

Horowitz, N.H., Hobby, G.L., and Hubbard, J.S. (1976) The Viking carbon assimilation experiments: interim report. Science 194:1321-1322.

Houghton, J.T., Ding, Y., Griggs, D.J., Noguer, M., van der Linden, P.J., Dai, X., Maskell, K., and Johnson, C.A., editors. (2001) Climate Change 2001: The Scientific Basis, Third Assessment Report of the International Panel on Climate Change (IPCC), Cambridge University Press, Cambridge, UK, Chapter 4.

Hsu, H.-W., Postberg, F., Sekine, Y., Shibuya, T., Kempf, S., Horányi, M., Juhász, A., Altobelli, N., Suzuki, K., Masaki, Y., Kuwatani, T., Tachibana, S., Sirono, S., Moragas-Klostermeyer, G., and Srama, R. (2015) Ongoing hydrothermal activities within Enceladus. Nature 519:207-210.

Ida, S. and Lin, D.N.C. (2004) Toward a deterministic model of planetary formation. I. A desert in the mass and semimajor axis distributions of extrasolar planets. Astrophys J 604:388-413.
Javaux, E.J. (2007) The early eukaryotic fossil record. $A d v$ Exp Med Biol 607:1-19.

Johnson, R.E., Famá, M., Liu, M., Baragiola, R.A., Sittler, E.C., and Smith, H.T. (2008) Sputtering of ice grains and icy satellites in Saturn's inner magnetosphere. Planet Space Sci 56:1238-1243.

Kalkowsky, E. (1908) Oolith und Stromatolith im norddeutschen Buntsandstein. Zeitschrift der deutschen geologischen Gesellschaft 60:68-125.

Kaltenegger, L., Traub, W.A., and Jucks, K.W. (2007) Spectral evolution of an Earth-like planet. Astrophys J 658:598-616.

Kasting, J.F., Whitmire, D.P., and Reynolds, R.T. (1993) Habitable zones around main sequence stars. Icarus 101:108-128.

Kauffman, S.A. (1993) The Origins of Order, Oxford University Press, Oxford, UK.

Kiang, N.Y., Siefert, J., Govindjee, and Blankenship, R.E. (2007) Spectral signatures of photosynthesis I: Review of Earth organisms. Astrobiology 7:222-251.

Klein, H.P. (1978) The Viking biological investigations: review and status. Orig Life 9:157-160.

Kleine, T., Palme, H., Mezger, K., and Halliday, A.N. (2005) Hf-W chronometry of lunar metals and the age and early differentiation of the Moon. Science 310:1671-1674.

Knoll, A.H. (1985) Exceptional preservation of photosynthetic microorganisms in silicified carbonate and peat. Philos Trans $R$ Soc Lond B Biol Sci 311, doi:10.1098/rstb.1985.0143.

Knoll, A.H. (2011) The multiple origins of complex multicellularity. Annu Rev Earth Planet Sci 39:217-239.

Kristjánsson, J.K. and Hreggvidsson, G.O. (1995) Ecology and habitats of extremophiles. World J Microbiol Biotechnol 11:17-25.

Ku, C., Nelson-Sathi, S., Roettger, M., Sousa, F.L., Lockhart, P.J., Bryant, D., Hazkani-Covo, E., McInerney, J.O., Landan, G.L., and Martin, W.F. (2015) Endosymbiotic origin and differential loss of eukaryotic genes. Nature 524:427-432.

Lammer, H., Bredehöft, J.H., Coustenis, A., Khodachenko, M.L., Kaltenegger, L., Grasset, O., Prieur, D., Raulin, F., Ehrenfreund, P., Yamauchi, M., Wahlund, J.-E., Grießmeier, J.-M., Stangl, G., Cockell, C.S., Kulikov, Yu.N., Grenfell, J.L., and Rauer, H. (2009) What makes a planet habitable? Astronomy and Astrophysics Review 17:181-249.

Lane, N. and Martin, W.F. (2012) The origin of membrane bioenergetics. Cell 151:1406-1416.

Le Romancer, M., Gaillard, M., Geslin, C., and Prieur, D. (2007) Viruses in extreme environments. In Life in Extreme Environments, edited by R. Amils, C. Ellis-Evans, and H. Hinghofer-Szalkay, Springer, Dordrecht, pp 99-113.

Lebrun, T., Massol, H., Chassefière, E., Davaille, A., Marcq, E., Sarda, P., Leblanc, F., and Brandais, G. (2013) Thermal evolution of an early magma ocean in interaction with the atmosphere. J Geophys Res 118:1-22.

Leconte, J., Forget, F., Charney, B., Wordsworth, R., Selsis, F., and Millour, E. (2013) 3D climate modeling of close-in land planets: circulation patterns, climate moist bistability and habitability. Astron Astrophys 554, doi:10.1051/0004-6361/201321042.

Lederberg, J. (1960) Exobiology: approaches to life beyond the Earth. Science 132:393-400.

Lever, M.A., Heuer, V.B., Morono, Y., Masui, N., Schmidt, F., Alperin, M.J., Inagaki, F., Hinrichs, K.-U., and Teske, A. (2010) Acetogenesis in deep subseafloor sediments of the Juan de Fuca Ridge flank: a synthesis of geochemical, thermodynamic, and gene-based evidence. Geomicrobiol J 27:183-211.

Levin, G.V. and Straat, P.A. (1976) Viking labeled release biology experiment: interim results. Science 194:1322-1329.

Levin, G.V. and Straat, P.A. (1979) Completion of the Viking labeled release experiment on Mars. J Mol Evol 14:167-183. 
Li, Z. and Nair, S.K. (2012) Quorum sensing: how bacteria can coordinate activity and synchronize their response to external signals. Protein Sci 21:1403-1417.

Lin, H.W., Abad, G.G., and Loeb, A. (2014) Detecting industrial pollutants in the atmospheres of Earth-like exoplanets. Astrophys J 792, doi:10.1088/2041-8205/792/1/L7.

Lineweaver, C.H., Fenner, Y., and Gibson, B.K. (2004) The galactic habitable zone and the age distribution of complex life in the Milky Way. Science 303:59-62.

Lyons, N.A. and Kolter, R. (2015) On the evolution of bacterial multicellularity. Curr Opin Microbiol 24:21-28.

Madigan, M.T., Martinko, J.M., Bender, K.S., Buckley, D.H., and Stahl, D.A. (2014) Brock Biology of Microorganisms, Benjamin Cummings, Boston.

Maher, B. (2008) Personal genomes: the case of the missing heritability. Nature 456:18-21.

Margulis, L. (1970) Origin of Eukaryotic Cells, Yale University Press, New Haven, CT.

Martin, W.F., Baross, J., Kelley, D., and Russell, M.J. (2008) Hydrothermal vents and the origin of life. Nat Rev Microbiol 6:805-814.

Martin, W.F., Sousa, F.L., and Lane, N. (2014) Energy at life's origin. Science 344:1092-1093.

Martins, Z., Price, M.C., Goldman, N., Sephton, M.A., and Burchell, M.J. (2013) Shock synthesis of amino acids from impacting cometary and icy planet surface analogues. Nat Geosci 6:1045-1049.

MASE. (2014) MASE: Mars Analogues for Space Exploration. Project supported by European Community's Seventh Framework Programme (FP7/2007-2013) under Grant Agreement $\mathrm{n}^{\circ}$ 607297. Available online at http://mase.esf.org

Maurette, M. (2006) Micrometeorites and the Mysteries of Our Origins, Springer, Berlin.

Mayor, M. and Queloz, D. (1995) A Jupiter-mass companion to a solar-type star. Nature 378:355-359.

McCollom, T.M. and Seewald, J.S. (2013) Serpentinites, hydrogen, and life. Elements 9:129-134.

McDermott, J.M., Seewald, J.S., German, C.R., and Sylva, S.P. (2015) Pathways for abiotic organic synthesis at submarine hydrothermal fields. Proc Natl Acad Sci USA 112:7668-7672.

McKay, C.P. and Smith, H.D. (2005) Possibilities for methanogenic life in liquid methane on the surface of Titan. Icarus 178:274-276.

Melezhik, V.A. (2006) Multiple causes of Earth's earliest global glaciation. Terra Nova 18:130-137.

MEPAG. (2015) Mars Science Goals, Objectives, Investigations, and Priorities: 2015 Active Version, white paper posted June, 2015, by the Mars Exploration Program Analysis Group (MEPAG). Available online at http://mepag.nasa.gov/ reports.cfm?expand=science

Mills, D.B., Ward, L.M., Jones, C., Sweeten, B., Forth, M., Treusch, A.H., and Canfield, D.E. (2014) Oxygen requirements of the earliest animals. Proc Natl Acad Sci USA 111:4168-4172.

Miot, J., Benzerara, K., and Kappler, A. (2014) Investigating microbe-mineral interactions: recent advances in X-ray and electron microscopy and redox-sensitive methods. Annu Rev Earth Planet Sci 42:271-289.

Mumma, M., Villanueva, G.L., Novak, R.E., Hewagama, T., Bonev, B.P., DiSanti, M.A., Mandell, A.M., and Smith, M.D. (2009) Strong release of methane on Mars in Northern Summer 2003. Science 323:1041-1045.

Navarro-González, R., Navarro, K.F., de la Rosa, J., Iñiguez, E., Molina, P., Miranda, L.D., Morales, P., Cienfuegos, E., Coll,
P., Raulin, F., Amils, R., and McKay, C.P. (2006) The limitations on organic detection in Mars-like soils by thermal volatilization-gas chromatography-MS and their implications for the Viking results. Proc Natl Acad Sci USA 103:16089-16094.

Neuman, Y. (2006) Cryptobiosis: a new theoretical perspective. Prog Biophys Mol Biol 92:258-267.

Nicholson, W.L., Munakata, N., Horneck, G., Melosh, H.J., and Setlow, P. (2000) Resistance of Bacillus endospores to extreme terrestrial and extraterrestrial environments. Microbiol Mol Biol Rev 64:548-572.

Nicholson, W.L., Ricco, A.J., Agasid, E., Beasley, C., DiazAguado, M., Ehrenfreund, P., Friedericks, C., Ghassemieh, S., Henschke, M., Hines, J.W., Kitts, C., Luzzi, E., Ly, D., Mai, N., Mancinelli, R., McIntyre, M., Minelli, G., Neumann, M., Parra, M., Piccini, M., Rasay, R.M., Ricks, R., Santos, O., Schooley, A., Squires, D., Timucin, L., Yost, B., and Young, A. (2011) The O/OREOS mission: first science data from the Space Environment Survivability of Living Organisms (SESLO) payload. Astrobiology 11:951-958.

Nisbet, E.G. and Wilks, M.E. (1988) Archaean stromatolite reef at Steep Rock Lake, Akitokan, Northwestern Ontario. In Reefs: Canada and Adjacent Areas, Memoir 13, edited by H.H.J. Geldsetzer, N.P. James, and G.E. Tebbutt, Canadian Society of Petroleum Geologists, Calgary, pp 89-92.

Noell, A.C., Ely, T., Bolser, D.K., Darrach, H., Hodyss, R., Johnson, P.V., Hein, J.D., and Ponce, A. (2015) Spectroscopy and viability of Bacillus subtilis spores after ultraviolet irradiation: implications for the detection of potential bacterial life on Europa. Astrobiology 15:20-31.

Noffke, N. (2009) The criteria for biogeneicity of microbially induced sedimentary structures (MISS) in Archean, sandy deposits. Earth-Science Reviews 96:173-180.

Noffke, N. (2015) Ancient sedimentary structures in the $<3.7$ Ga Gillespie Lake Member, Mars, that resemble macroscopic morphology, spatial associations, and temporal succession in terrestrial microbialites. Astrobiology 15:169-192.

Öberg, K.I., Guzmán, V.V., Furuya, K., Qi, C., Aikawa, Y., Andrews, S.M., Loomis, R., and Wilner, D.J. (2015) The comet-like composition of a protoplanetary disk as revealed by complex cyanides. Nature 520:198-201.

Oehler, D.Z. and Cady, S.L. (2014) Biogenicity and syngeneity of organic matter in ancient sedimentary rocks: recent advances in the search for evidence of past life. Challenges 5:260-283.

Ohtomo, Y., Kakegawa, T., Ishida, A., Nagase, T., and Rosing, M.T. (2013) Evidence for biogenic graphite in early Archaean Isua metasedimentary rocks. Nat Geosci 7:25-28.

Onofri, S., de la Torre, R., de Vera, J.P., Ott, S., Zucconi, L., Selbmann, L., Scalzi, G., Venkateswaran, K.J., Rabbow, E., Sánchez Iñigo, F.J., and Horneck, G. (2012) Survival of rockcolonizing organisms after 1.5 years in outer space. Astrobiology 12:508-516.

Orange, F., Westall, F., Disnar, J.-R., Prieur, D., Bienvenu, N., Le Romancer, M., and Défarge, Ch. (2009) Experimental silicification of the extremophilic Archaea Pyroccus abyssi and Methanocaldococcus jannaschii. Applications in the search for evidence of life in early Earth and extraterrestrial rocks. Geobiology 7:403-418.

Parfrey, L.W., Lahr, D.J.G., Knoll, A.H., and Katz, L.A. (2011) Estimating the timing of early eukaryotic diversification with multigene molecular clocks. Proc Natl Acad Sci USA 108:13624-13629.

Parnell, J., Cullen, D., Sims, M.R., Bowden, S., Cockell, C.S., Court, R., Ehrenfreund, P., Gaubert, F., Grant, W., Parro, V., 
Rohmer, M., Sephton, M., Stan-Lotter, H., Steele, A., Toporski, J., and Vago, J. (2007) Searching for life on Mars: selection of molecular targets for ESA's Aurora ExoMars mission. Astrobiology 7:578-604.

Plainaki, C., Milillo, A., Massetti, S., Mura, A., Jia, X., Orsini, S., Mangano, V., De Angelis, E., and Rispoli, R. (2015) The $\mathrm{H}_{2} \mathrm{O}$ and $\mathrm{O}_{2}$ exospheres of Ganymede: the result of a complex interaction between the jovian magnetospheric ions and the icy moon. Icarus 245:306-319.

Pogoda de la Vega, U., Rettberg, P., and Reitz, G. (2007) Simulation of the environmental climate conditions on martian surface and its effect on Deinococcus radiodurans. Adv Space Res 40:1672-1677.

Pollack, J.B., Hubickyj, O., Bodenheimer, P., Lissauer, J.J., Podolak, M., and Greenzweig, Y. (1996) Formation of the giant planets by concurrent accretion of solids and gas. Icarus 124:62-85.

Postberg, F., Schmidt, J., Hillier, J., Kempf, S., and Srama, R. (2011) A salt-water reservoir as the source of a compositionally stratified plume on Enceladus. Nature 474:620-622.

Proskurowski, G., Lilley, M.D., Seewald, J.S., Früh-Green, G.L., Olson, E.J., Lupton, J.E., Sylva, S.P., and Kelley, D.S. (2008) Abiogenic hydrocarbon production at Lost City hydrothermal field. Science 319:604-607.

Pross, A. (2012) What is Life? How Chemistry Becomes Biology, Oxford University Press, Oxford, UK.

Rabbow, E., Rettberg, P., Barczyk, S., Bohmeier, M., Parpart, A., Panitz, C., Horneck, G., Burfeindt, J., Molter, F., Jaramillo, E., Pereira, C., Weiß, P., Willnecker, R., Demets, R., and Dettmann, J. (2015) The astrobiological mission EXPOSE-R on board of the International Space Station. International Journal of Astrobiology 14:3-16.

Ramamurthy, T., Ghosh, A., Pazhani, G.P., and Shinoda, S. (2014) Current perspectives on viable but non-culturable (VBNC) pathogenic bacteria. Front Public Health 2, doi:10.3389/fpubh.2014.00103.

Rauer, H., Gebauer, S., von Paris, P., Cabrera, J., Godolt, M., Grenfell, J.L., Belu, A., Selsis, F., Hedelt, P., and Schreier, F. (2011) Potential biosignatures in super-Earth atmospheres I. Spectral appearance of super-Earths around M dwarfs. Astron Astrophys 529, doi:10.1051/0004-6361/201014368.

Relman, D.A. (2008) 'Til death do us part': coming to terms with symbiotic relationships. Nat Rev Microbiol 6:721-724.

Riding, R. (2011) The history and nature of stromatolites. The nature of stromatolites: 3,500 million years of history and a century of research. In Advances in Stromatolite Geobiology, Lecture Notes in Earth Sciences Vol. 131, edited by J. Reitner, N.-V. Quéric, and G. Arp, Springer, Berlin, pp 29-74.

Roldan, A., Hollingsworth, N., Roffey, A., Islam, H.-U., Goodall, J.B.M., Catlow, C.R.A., Darr, J.A., Bras, W., Sankar, G., Holt, K.B., Hogarth, G., and de Leeuw, N.H. (2015) Bio-inspired $\mathrm{CO}_{2}$ conversion by iron sulfide catalysts under sustainable conditions. Chem Commun 51:7501-7504.

Röling, W.F.M., Aerts, J.W., Patty, C.H.L., ten Kate, I.L., Ehrenfreund, P., and Direito, S.O.L. (2015) The significance of microbe-mineral-biomarker interactions in the detection of life on Mars and beyond. Astrobiology 15:492-507.

Rosa, P., Ehrenfreund, P., Horneck, G., and Thiele, G. (2013) European space research in support of international partnership. Space Policy 29:234-237.

Roth, L., Saur, J., Retherford, K.D., Strobel, D.F., Feldman, P.D., McGrath, M.A., and Nimmo, F. (2013) Transient water vapor at Europa's south pole. Science 343:171-174.

Rothschild, L.J. and Mancinelli, R. (2001) Life in extreme environments. Nature 409:1092-1101.
Russell, M.J. and Hall, A.J. (1997) The emergence of life from iron monosulphide bubbles at a submarine hydrothermal redox and pH front. J Geol Soc London 154:377-402.

Safronov, V. (1969) Evolution of Protoplanetary Cloud and Formation of the Earth and Planets, Nauka, Moscow.

Sagan, C. and Mullen, G. (1972) Earth and Mars: evolution of atmospheres and surface temperatures. Science 177:52-56.

Saladino, R., Botta, G., Pino, S., Costanzo, G., and Di Mauro, E. (2012) Genetics first or metabolism first? The formamide clue. Chem Soc Rev 41:5526-5565.

Saladino, R., Botta, G., Delfino, M., and Di Mauro, E. (2013) Meteorites as catalysts for prebiotic chemistry. Chemistry 19:16916-16922.

Saladino, R., Carota, E., Botta, G., Kapralov, M., Timoshenko, G.N., Rozanov, A.Y., Krasavin, E., and Di Mauro, E. (2015) Meteorite-catalyzed syntheses of nucleosides and of other prebiotic compounds from formamide under proton irradiation. Proc Natl Acad Sci USA 112:7109-7110.

Schmitt-Kopplin, P., Gabelica, Z., Gougeon, R.D., Fekete, A., Kanawati, B., Harir, M., Gebefuegi, I., Eckel, G., and Hertkorn, N. (2010) High molecular diversity of extraterrestrial organic matter in the Murchison meteorite was revealed 40 years after it fell. Proc Natl Acad Sci USA 107:2763-2768.

Schrenk, M.O., Brazelton, W.J., and Lang, S.Q. (2013) Serpentinization, carbon and deep life. Reviews in Mineralogy and Geochemistry 75:575-606.

Schrödinger, E. (1944) What is Life? Cambridge University Press, Cambridge, UK.

Schuchmann, K. and Müller, V. (2014) Autotrophy at the thermodynamic limit of life: a model for energy conservation in acetogenic bacteria. Nat Rev Microbiol 12:809-821.

Segura, A., Krelove, K., Kasting, J.F., Sommerlatt, D., Meadows, V., Crisp, D., Cohen, M., and Mlawer, E. (2003) Ozone concentrations and ultraviolet fluxes on Earth-like planets around other stars. Astrobiology 3:689-708.

Shields, A., Meadows, V., Bitz, C., Pierrehumbert, R., Joshi, M., and Robinson, T. (2013) The effect of host star spectral energy distribution and ice-albedo feedback on the climate of extrasolar planets. Astrobiology 13:715-739.

Shimkets, L.J. (2013) Prokaryotic life cycles. In The Prokaryotes, edited by E. Rosenberg, E.F. DeLong, S. Lory, E. Stackebrandt, and F. Thompson, Springer, Berlin, pp 317-336.

Shock, E.L., McCollom, T.M., and Schulte, M.D. (1998) The emergence of metabolism from within hydrothermal systems. In Thermophiles: The Keys to Molecular Evolution and the Origin of Life, edited by J. Wiegel and M.W.W. Adams, Taylor \& Francis, London, pp 59-76.

Simakov, M.B. (2008) Asteroids and the origin of life-two steps of chemical evolution on the surface of these objects. Earth, Planets and Space 60:75-82.

Singh, O.V. (2013) Extremophiles: Sustainable Resources and Biotechnological Implications, John Wiley \& Sons, Hoboken, NJ.

Sotin, C. and Prieur, D. (2007) Jupiter's moon Europa: geology and habitability. In Complete Course in Astrobiology, edited by G. Horneck and P. Rettberg, Wiley-VCH, Weinheim, pp 253-271. Sparks, W.B., Hough, J.H., and Bergeron, L.E. (2005) A search for chiral signatures on Mars. Astrobiology 5:737-748.

Stan-Lotter, H. (2007) Extremophiles: the physicochemical limits of life (growth and survival). In Complete Course in Astrobiology, edited by G. Horneck and P. Rettberg, WileyVCH, Weinheim, pp 121-150.

Stevenson, A., Cray, J.A., Williams, J.P., Santos, R., Sahay, R., Neuenkirchen, N., McClure, C.D., Grant, I.R., Houghton, J.D., Quinn, J.P., Timson, D.J., Patil, S.V., Singhal, R.S., Antón, J., 
Dijksterhuis, J., Hocking, A.D., Lievens, B., Rangel, D.E., Voytek, M.A., Gunde-Cimerman, N., Oren, A., Timmis, K.N., McGenity, T.J., and Hallsworth, J.E. (2015) Is there a common water-activity limit for the three domains of life? ISME J 9:1333-1351.

Stüeken, E.E., Anderson, R.E., Bowman, J.S., Brazelton, W.J., Colangelo-Lillis, J., Goldman, A.D., Som, S.M., and Baross, J.A. (2013) Did life originate from a global chemical reactor? Geobiology 11:101-126.

Szathmáry, E. and Demeter, L. (1987) Group selection of early replicators and the origin of life. J Theor Biol 128:463-486.

Tarter, J.C., Backus, P.R., Mancinelli, R.L., Aurnou, J.M., Backman, D.E., Basri, G.S., Boss, A.P., Clarke, A., Deming, D., Doyle, L.R., Feigelson, E.D, Freund, F., Grinspoon, D.H., Haberle, R.M., Hauck, S.A., II, Heath, M.J., Henry, T.J., Hollingsworth, J.L., Joshi, M.M., Kilston, S., Liu, M.C., Meikle, E., Reid, I.N., Rothschild, L.J., Scalo, J., Segura, A., Tang, C.M., Tiedje, J.M., Turnbull, M.C., Walkowicz, L.M., Weber, A.L., and Young, R.E. (2007) A reappraisal of the habitability of planets around M dwarf stars. Astrobiology 7:30-65.

Tera, F., Papanastassiou, D.A., and Wasserburg, G.J. (1974) Isotopic evidence for a terminal lunar cataclysm. Earth Planet Sci Lett 22:1-21.

Thomas, C.M. and Nielsen, K.M. (2005) Mechanisms of, and barriers to, horizontal gene transfer between bacteria. Nat Rev Microbiol 3:711-721.

Tice, M.M. and Lowe, D.R. (2004) Photosynthetic microbial mats in the 3,416-Myr-old ocean. Nature 431:549-552.

Tsiganis, K., Gomes, R., Morbidelli, A., and Levison, H.F. (2005) Origin of the orbital architecture of the giant planets of the Solar System. Nature 435:459-461.

Ueno, Y., Yamada, K., Yoshida, N., Maruyama, S., and Isozaki, Y. (2006) Evidence from fluid inclusions for microbial methanogenesis in the early Archaean era. Nature 440:516-519.

Vago, J. and Kminek, G. (2007) Putting together an exobiology mission: the ExoMars example. In Complete Course in Astrobiology, edited by G. Horneck and P. Rettberg, WileyVCH, Weinheim, pp 321-351.

Vago, J., Gardini, B., Kminek, G., Baglioni, P., Gianfiglio, G., Santovincenzo, A., Bayón, S., and van Winnendael, M. (2006) ExoMars: searching for life on the Red Planet. ESA Bull 126:17-23.

Vago, J., Witasse, O., Svedhem, H., Baglioni, P., Haldemann, A., Gianfiglio, G., Blancquaert, T., McCoy, D., and de Groot, R. (2015) ESA ExoMars program: the next step in exploring Mars. Solar System Research 49:518-528.

Vaidya, N., Manapat, M.L., Chen, I.A., Xulvi-Brunet, R., Hayden, E.J., and Lehman, N. (2012) Spontaneous network formation among cooperative RNA replicators. Nature 491:72-77.

Van Kranendonk, M.J., Smithies, R.H., Griffin, W.L., Huston, D.L., Hickman, A.H., Champion, D.C., Anhaeusser, C.R., and Pirajno, F. (2015) Making it thick: a volcanic plateau origin of Palaeoarchean continental lithosphere of the Pilbara and Kaapvaal cratons. In Continent Formation through Time, Geological Society Special Publication 389, edited by N.M.W. Roberts, M. Van Kranendonk, S. Parman, S. Bottome Shirey, and P.D. Clift, The Geological Society, London, pp 83-111.

Vergne, J., Cottin, H., da Silva, L., Brack, A., Chaput, D., and Maurel, M.-C. (2015) The AMINO experiment: RNA stability under solar radiation studied on the EXPOSE-R facility of the International Space Station. International Journal of Astrobiology 14:99-103.

Wächtershäuser, G. (2006) From volcanic origins of chemoautotrophic life to Bacteria, Archaea and Eukarya. Philos Trans R Soc Lond B Biol Sci 361:1787-1806.
Walsh, K.J., Morbidelli, A., Raymond, S.N., O'Brien, D.P., and Mandell, A.M. (2011) A low mass for Mars from Jupiter's early gas-driven migration. Nature 475:206-209.

Walsh, M.M. (1992) Microfossils and possible microfossils from the early Archean Onverwacht Group, Barberton Mountain Land, South Africa. Precambrian Res 54:271-293.

Wassmann, M., Moeller, R., Reitz, G., and Rettberg, P. (2010) Adaptation of Bacillus subtilis cells to Archean-like UV climate: relevant hints of microbial evolution to remarkably increased radiation resistance. Astrobiology 10:605-615.

Wassmann, M., Moeller, R., Rabbow, E., Panitz, C., Horneck, G., Reitz, G., Douki, Th., Cadet, J., Stan-Lotter, H., Cockell, C.S., and Rettberg, P. (2012) Survival of spores of the UVresistant Bacillus subtilis strain MW01 after exposure to lowEarth orbit and simulated martian conditions: data from the space experiment ADAPT on EXPOSE-E. Astrobiology 12:498-507.

Webster, C.R., Mahaffy, P.R., Atreya, S.K., Flesch, G.J., Mischna, M.A., Meslin, P.-Y., Farley, K.A., Conrad, P.G., Christensen, L.E., Pavlov, A.A., Martín-Torres, J., Zorzano, M.-P., McConnochie, T.H., Owen, T., Eigenbrode, J.L., Glavin, D.P., Steele, A., Malespin, C.A., Archer, P.D., Jr., Sutter, B., Coll, P., Freissinet, C., McKay, C.P., Moores, J.E., Schwenzer, S.P., Bridges, J.C., Navarro-González, R., Gellert, R., Lemmon, M.T., the MSL Science Team. (2015) Mars methane detection and variability at Gale Crater. Science 347:415-417.

Westall, F. (2011) Early life. In Origins and Evolution of Life: An Astrobiology Perspective, edited by M. Gargaud, P. López-Garcia, and H. Martin, Cambridge University Press, Cambridge, UK, pp 391-413.

Westall, F. (2012) The early Earth. In Frontiers of Astrobiology, edited by C. Impey, J.I. Lunine, and J.G. Funes, Cambridge University Press, Cambridge, UK, pp 89-114.

Westall, F. and Cavalazzi, B. (2011) Biosignatures in rocks. In Encyclopedia of Geobiology, edited by V. Thiel and J. Reitner, Springer, Berlin, pp 189-201.

Westall, F. and Southam, G. (2006) Early life on Earth. In Archean Geodynamics and Environments, Geophysical Monograph 164, edited by K. Benn, J.-C. Mareschal, and K.C. Condie, American Geophysical Union, Washington, DC, pp 283-304.

Westall, F., de Vries, S.T., Nijman, W., Rouchon, V., Orberger, B., Pearson, V., Watson, J., Verchovsky, A., Wright, I., Rouzaud, J.-N., Marchesini, D., and Anne, S. (2006a) The 3.466 Ga Kitty's Gap chert, an Early Archean microbial ecosystem. In Processes on the Early Earth, Geological Society of America Special Paper 405, edited by W.U. Reimold and R.L. Gibson, Geological Society of America, Boulder, CO, pp 105-131.

Westall, F., de Ronde, C.E.J., Southam, G., Grassineau, N., Colas, M., Cockell, C., and Lammer, H. (2006b) Implications of a 3.472-3.333 Ga-old subaerial microbial mat from the Barberton greenstone belt, South Africa for the UV environmental conditions on the early Earth. Philos Trans $R$ Soc Lond B Biol Sci 361:1857-1875.

Westall, F., Cavalazzi, B., Lemelle, L., Marrocchi, Y., Rouzaud, J.N., Simionovici, A., Salomé, M., Mostefaoui, S., Andreazza, C., Foucher, F., Toporski, J., Jauss, A., Thiel, V., Southam, G., Mac-Lean, L., Wirick, S., Hofmann, A., Meibom, A., Robert, F., and Défarge, C. (2011) Implications of in situ calcification for photosynthesis in a $\sim 3.3$ Ga-old microbial biofilm from the Barberton greenstone belt, South Africa. Earth Planet Sci Lett 310:468-479.

Westall, F., Loizeau, D., Foucher, F., Bost, N., Bertrand, M., Vago, J., and Kminek, G. (2013) Habitability on Mars from a microbial point of view. Astrobiology 13:887-897. 
Westall, F., Campbell, K.A., Bréhéret, J.G., Foucher, F., Gautret, P., Hubert, A., Sorieul, S., Grassineau, N., and Guido, D.M. (2015a) Archean $(3.33 \mathrm{Ga})$ microbe-sediment systems were diverse and flourished in a hydrothermal context. Geology 43:615-618.

Westall, F., Foucher, F., Bost, N., Bertrand, M., Loizeau, D., Vago, J.L., Kminek, G., Gaboyer, F., Campbell, K.A., Bréhéret, J.-G., Gautret, P., and Cockell, C.S. (2015b) Biosignatures on Mars: what, where, and how? Implications for the search for martian life. Astrobiology 15:998-1029.

Wilde, S.A., Valley, J.W., Peck, W.H., and Graham, C.M. (2001) Evidence from detrital zircons for the existence of continental crust and oceans on the Earth 4.4 Gyr ago. Nature 409:175-178.

Wyatt, M.C., Kennedy, G., Sibthorpe, B., Moro-Martin, A., Lestrade, J.-F., Ivison, R.J., Matthews, B., Udry, S., Greaves, J.S., Kalas, P., Lawler, S., Su, K.Y.L., Rieke, G.H., Booth, M., Bryden, G., Horner, J., Kavelaars, J.J., and Wilner, D. (2012) Herschel imaging of 61 Vir: implications for the prevalence of debris in low-mass planetary systems. Mon Not $R$ Astron Soc 424:1206-1223.

Yen, A.S., Kim, S.S., Hecht, M.H., Frant, M.S., and Murray, B. (2000) Evidence that the reactivity of the martian soil is due to superoxide ions. Science 289:1909-1912.

Zahnle, K., Freedman, R.S., and Catling, D.C. (2011) Is there methane on Mars? Icarus 212:493-503.

Zahnle, K.J., Kasting, J.F., and Pollack, J.B. (1988) Evolution of a steam atmosphere during Earth's accretion. Icarus 74:62-97.

Zheng, W., Rasmussen, U., Zhen, S., Chen, B., Gao, Y., Guan, X., Larsson, J., and Bergman, B. (2013) Multiple modes of cell death discovered in a prokaryotic (cyanobacterial) endosymbiont. PLoS One doi:10.1371/journal.pone.0066147.

Zimorski, V., Ku, C., Martin, W.F., and Gould, S.B. (2014) Endosymbiotic theory for organelle origins. Curr Opin $\mathrm{Mi}$ crobiol 22:38-48.

\begin{tabular}{|c|}
\hline 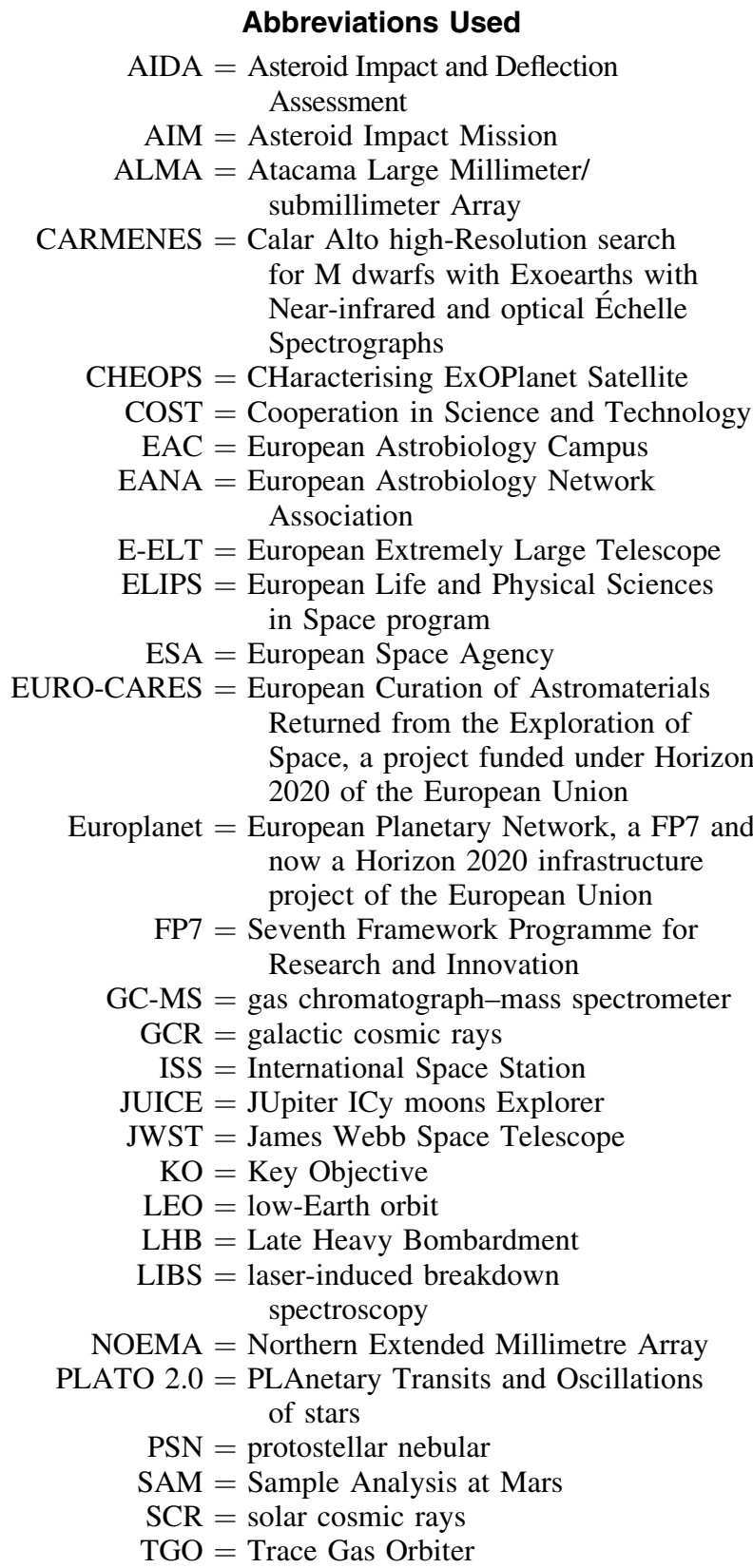 \\
\hline
\end{tabular}

